\title{
Zero-Free Regions for Dirichlet L-Functions, and the Least Prime in an Arithmetic Progression
}

\author{
D.R. Heath-Brown \\ Magdalen College, Oxford OX1 4AU
}

\section{Introduction and Statement of Results}

The classical theorem of Dirichlet states that any arithmetic progression $a(\bmod q)$ in which $a$ and $q$ are relatively prime contains infinitely many prime numbers. A natural question to ask is then, how big is the first such prime, $P(a, q)$ say? In one direction we have trivially

$$
\max _{a} P(a, q) \geq(1+o(1)) \phi(q) \log q
$$

since the number of primes below $(1-\delta) \phi(q) \log q$ will be less than $\phi(q)$ if $q$ is large enough. Pomerance [33] showed that the factor $1+o(1)$ can be replaced by $e^{\gamma}+o(1)$. Moreover if $q$ has at most

$$
\exp (\log \log q / \log \log \log q)
$$

prime factors, as happens for "almost all" $q$, he showed that the right hand side of (1.1) may be replaced by

$$
\left(e^{\gamma}+o(1)\right) \phi(q) \log q \frac{(\log \log q)(\log \log \log \log q)}{(\log \log \log q)^{2}} .
$$

It has been conjectured by Granville and Pomerance [12] that

$$
\max _{a} P(a, q) \gg \phi(q) \log ^{2} q
$$

for all $q$.

As regards upper bounds, the most important result is that of Linnik [24], [25], who proved that

$$
P(a, q) \ll q^{L}
$$

for some absolute constant $L$. On the Generalized Riemann Hypothesis one can in fact show that

$$
P(a, q) \ll \phi(q)^{2} \log ^{2} q
$$




\begin{tabular}{rrll}
\multicolumn{1}{c}{ L } & Date & Author & Reference \\
\hline 10000 & 1957 & Pan & {$[30]$} \\
5448 & 1958 & Pan & {$[31]$} \\
777 & 1965 & Chen & {$[3]$} \\
630 & 1971 & Jutila & {$[39]^{1}$} \\
550 & 1970 & Jutila & {$[20]$} \\
168 & 1977 & Chen & {$[4]$} \\
80 & 1977 & Jutila & {$[21]$} \\
36 & 1977 & Graham & {$[9]$} \\
20 & 1981 & Graham & {$[11]$} \\
17 & 1979 & Chen & {$[5]$} \\
16 & 1986 & Wang \& Liu & {$[40]$} \\
13.5 & 1989 & Chen \& Liu & {$[7]$}
\end{tabular}

Table 1: Estimates for Linnik's Constant

A very slightly weaker result follows from a remark of Hooley [16]. This falls just short of the conjecture that $P(a, q) \leq q^{2}$ for all $q \geq 2$.

In the light of the above remarks it is of interest to know admissable values for the constant $L$ in Linnik's Theorem. This question has been addressed by a number of authors, and the results are displayed in Table 1. In this connection it should be pointed out that even though Chen's paper [5] appears to antedate that of Graham [11], Chen's work was based on a preprint of Graham's - although this is not apparent from the references cited by Chen.

Treatments of Linnik's Theorem depend on three main principles. The first of these is the zero-free region for Dirichlet L-functions (Gronwall [13], Landau [23] and Titchmarsh [35]):-

Principle 1 There is an effectively computable positive constant $c_{1}$ such that

$$
\prod_{\chi(\bmod q)} L(s, \chi)
$$

has at most one zero in the region

$$
\sigma \geq 1-\frac{c_{1}}{\log q(2+|t|)} .
$$

Such a zero, if it exists, is real and simple, and corresponds to a non-principal real character.

The second principle due to Linnik [25], is the "Deuring-Heilbronn" phenomenon:-

\footnotetext{
${ }^{1}$ This paper of Turán quotes Jutila's unpublished result - see page 370.
} 
Principle 2 There is an effectively computable positive constant $c_{2}$ such that, if the exceptional zero in Principle 1 exists, and is $1-\delta /(\log q)$ say, then the function (1.2) has no other zeros in the region

$$
\sigma \geq 1-\frac{c_{2}\left(\log \delta^{-1}\right)}{\log q(2+|t|)} .
$$

The third principle, also due to Linnik [24], is the "log-free" zero-density estimate:-

Principle 3 There is an effectively computable positive constant $c_{3}$ such that

$$
\sum_{\chi(\bmod q)} N(\sigma, T, \chi) \ll(q T)^{c_{3}(1-\sigma)} \quad(T \geq 1) .
$$

In fact Linnik's versions of the latter two estimates were restricted to small ranges of $t$ and $T$, but this is inessential.

It is perhaps appropriate to mention here the treatment of Linnik's Theorem given by Motohashi [29]. This avoids the use of Principles 2 and 3. Instead it feeds the ideas behind their proofs directly into the estimation of a prime number sum, without mentioning zeros of L-functions.

Principles 1, 2 and 3 have been established in a variety of ways. Linnik's original treatment was considerably simplified by the introduction of Turán's power sum method (see Turán [38] and Knapowski [22]). A further round of simplification was initiated by Selberg's "pseudo-characters" (see Motohashi [28]). Perhaps the other most important development was Graham's generalization [9] of Principle 1 to give wider regions containing at most 4 zeros, say.

A number of forms of the above results have been given, with various constraints on $t, T$ and the characters $\chi$. The strongest versions comparable with the results of the present paper are as follows.

The estimate of Principle 1 holds with $c_{1}=0.10367$, if $q$ is large enough.

Graham [11] attributes this to unpublished work of Schoenfeld. There is a proof in Chen [6; Lemma 10]. See also McCurley [26] and Stechkin [34].

If $q$ is sufficiently large, the function (1.2) has at most 2 distinct zeros in the region

$$
\sigma \geq 1-\frac{0.2069}{\log q(2+|t|)},
$$

and at most 4 distinct zeros in the region

$$
\sigma \geq 1-\frac{0.2769}{\log q(2+|t|)} .
$$

This is due to Graham [11; Theorems 2 and 3].

The estimate of Principle 2 holds for any $c_{2}<\frac{2}{3}$, providing that $q$ is large enough and $\delta$ is small enough. 
For this see Graham [9].

Let $N(\lambda)$ be the the number of $L$-functions modulo $q$ which have a zero in the region

$$
\sigma \geq 1-\frac{\lambda}{\log q}, \quad|t| \leq 1 .
$$

Then

$$
N(\lambda) \leq \frac{6.2557}{\lambda} e^{3.5 \lambda}
$$

for $\lambda \leq \log \log \log q$, and $q$ sufficiently large.

This follows from Theorem 1 of Chen [5].

For comparison, we shall prove as follows.

Theorem 1 If $q$ is sufficiently large the function (1.2) has at most one zero in the region

$$
\sigma \geq 1-\frac{0.348}{\log q}, \quad|t| \leq 1 .
$$

Such a zero, if it exists, is real and simple, and corresponds to a non-principal real character.

Theorem 2 If $q$ is sufficiently large, the function (1.2) has at most 2 zeros, counted according to multiplicity, in the region

$$
\sigma \geq 1-\frac{0.696}{\log q},|t| \leq 1 .
$$

Moreover, for large enough $q$, there exists a character $\chi_{1}(\bmod q)$ such that $L(s, \chi)$ is non-vanishing for

$$
\sigma \geq 1-\frac{0.702}{\log q}, \quad|t| \leq 1
$$

for all characters $\chi(\bmod q)$ with $\chi \neq \chi_{1}, \overline{\chi_{1}}$.

Theorem 3 If $q$ is sufficiently large, there are characters $\chi_{1}, \chi_{2}(\bmod q)$ such that $L(s, \chi)$ is non-vanishing for

$$
\sigma \geq 1-\frac{0.857}{\log q}, \quad|t| \leq 1
$$

for all characters $\chi(\bmod q)$ with $\chi \neq \chi_{1}, \overline{\chi_{1}}, \chi_{2}, \overline{\chi_{2}}$.

Theorem 4 Let $\varepsilon>0$ be given. Suppose that $\chi$ is a real non-principal character modulo q, and that

$$
L\left(1-\frac{\lambda}{\log q}, \chi\right)=0,
$$


with $\lambda \leq 0.348$. Then, if $q$ is sufficiently large, the function (1.2) has only the zero $s=1-\lambda / \log q$ in the region

$$
\sigma \geq 1-\frac{\min \left\{\left(\frac{12}{11}-\varepsilon\right) \log \frac{1}{\lambda}, \frac{1}{3} \log \log \log q\right\}}{\log q} .
$$

Theorem 5 Let $\varepsilon>0$ be given. Then, if $q$ is sufficiently large, we have

$$
N(\lambda) \leq(1+\varepsilon) \frac{67}{6 \lambda}\left(e^{73 \lambda / 30}-e^{16 \lambda / 15}\right)
$$

for $\lambda \leq \frac{1}{3} \log \log \log q$.

Clearly Theorems 1-4 are significant improvements over what was known previously. By contrast Theorem 5 is better solely because we make use of new bounds for character sums, due to Burgess [2], which were unavailable to previous authors.

We may remark at this point that Pintz [32] has considered the situation in which Principle 1 is restricted to real zeros of a single real L-function. His line of attack is quite different from ours, and leads to the value $c_{1}=4+o(1)$, which is appreciably better than our estimate $2.427 \ldots$, given by Lemma 8.2. This suggests that Theorems 1-3 may be capable of further improvement.

We also observe that the constant 0.857 in Theorem 3 is in fact $6 / 7+o(1)$. This is indeed rather curious, since in general the constants produced by our proofs have every appearance of being transcendental.

We apply our results to the estimation of the constant in Linnik's Theorem.

Theorem 6 We have $P(a, q) \ll q^{5.5}$.

Our exponent 5.5 should be compared with the previous best, namely 13.5 , due to Chen and Liu [7], and also with the exponent $2+\varepsilon$ which one obtains on the Generalized Riemann Hypothesis. We may also point out that if the constant $c_{1}$ in Principle 1 can be taken arbitrarily large, and if the exceptional zero does not exist, then one easily obtains a bound

$$
P(a, q) \ll_{\varepsilon} q^{2.4+\varepsilon},
$$

from the estimate

$$
\sum_{\chi(\bmod q)} N(\sigma, T, \chi) \ll_{\varepsilon}(q T)^{\left(\frac{12}{5}+\varepsilon\right)(1-\sigma)}, \quad(T \geq 1) .
$$

The latter is a combination of a log-free bound of Jutila [21] with a result of Huxley [17]. In this way Iwaniec [18] established the bound (1.3) for those $q$ composed of any fixed set of prime divisors. 
Another conditional result, which will be of importance to us, is that of Heath-Brown [14]. Here one assumes that the exceptional zero of Principle 1 does indeed exist, and is $1-\lambda / \log q$ say. One then has

$$
P(a, q) \ll_{\varepsilon} q^{3+\varepsilon},
$$

providing that $\lambda \leq \lambda(\varepsilon)$. It follows that, for the purposes of proving Theorem 6 , one may assume that $\lambda \gg 1$. This is useful, since the order of magnitude of $\lambda$ is then constant. Moreover the result of Heath-Brown [14] is effective, so that the constant in our Theorem 6 is also effectively computable.

It should be noted that our work makes crucial use of Burgess's bounds [1], [2] for L-functions. Indeed one reason for our improvements is that we are able to bring these into play, as in $\S 3$, where other authors had failed. Burgess obtains better bounds for cube-free moduli than for the general case, and it follows that all our work could be improved if the modulus $q$ was assumed to be cube-free. Unfortunately it is by no means clear, without a complete reworking of this paper, just what exponent one would achieve in Theorem 6 , for example, if $q$ were assumed to be cube-free.

The various techniques involved in the proofs will be discussed in detail in the relevant sections. However we may remark here that the arguments rely heavily on numerical calculations. These we do not reproduce in full, nor have we attempted any rigorous analysis of the rounding and truncation errors in the computer algorithms employed. Indeed we take the view that the precise value of the constants obtained is unimportant; what matters is their clear superiority over those contained in previous works. At this stage only one point of notation need be specified: we shall write

$$
\log q=\mathcal{L}
$$

the parameter $\mathcal{L}$ being of extremely frequent occurence.

Finally it is a pleasure to record my thanks to Dr Wang Wei, who started my interest in these problems. 


\section{Burgess's Bounds}

In this section we consider bounds for Dirichlet L-functions. We take as our starting point the following estimates due to Burgess [1], [2].

Lemma 2.1 Let $q \geq 1$ and let $\chi$ be a primitive character modulo $q$. Let $N \geq 1$ and $1 \leq H \leq q$. Then for any $\varepsilon>0$ we have

$$
\sum_{N<n \leq N+H} \chi(n) \ll_{\varepsilon, k} q^{(k+1) /\left(4 k^{2}\right)+\varepsilon} H^{1-1 / k}
$$

for $k=2$ or 3 . Moreover if $q$ is cube-free the estimate (2.1) holds for any $k \in \mathbb{N}$.

Actually Burgess's formulation refers to characters which are non-principal, but not necessarily primitive. Of course (2.1) holds trivially when $1 \leq H \leq q$ and $\chi$ is identically 1.

In order to cover the case in which $q$ is not quite cube-free we may use the following lemma to factorize $\chi$.

Lemma 2.2 Let $q=u v$ with $(u, v)=1$. If $\chi$ is a primitive character modulo $q$, then there exist primitive characters $\chi_{u}$ and $\chi_{v}$, to moduli $u$ and $v$ respectively, such that $\chi=\chi_{u} \chi_{v}$. Moreover, the orders of $\chi_{u}$ and $\chi_{v}$ will divide the order of $\chi$.

For the proof one sets

$$
\chi_{u}(n)=\chi(n+u \bar{u}(1-n))
$$

where $u \bar{u} \equiv 1(\bmod v)$, and similarly for $\chi_{v}$. The required properties now follow from the observation that $\chi_{u}(n)=\chi\left(n_{u}\right)$ with

$$
n_{u} \equiv n(\bmod u), \quad n_{u} \equiv 1(\bmod v) .
$$

We now suppose that $\chi$ is primitive to modulus $q$, and we write $q=u v$, where $u$ is the product of those factors $p^{e} \| q$ for which $e \leq 2$. If $n \in(N, N+H]$ and $n \equiv a(\bmod v)$ we may set $n=a+v s$ where

$$
\frac{N-a}{v}<s \leq \frac{N-a}{v}+\frac{H}{v} .
$$

We then have

$$
\chi(n)=\chi_{u}(n) \chi_{v}(n)=\chi_{u}(a+v s) \chi_{v}(a)=\chi_{u}(\bar{v} a+s) \chi_{u}(v) \chi_{v}(a),
$$

where $v \bar{v} \equiv 1(\bmod u)$ as before. We now write $t=\bar{v} a+s$ and

$$
I(a)=\left(\frac{N-a}{v}+\bar{v} a, \frac{N-a}{v}+\bar{v} a+\frac{H}{v}\right] .
$$


It therefore follows from Lemma 2.1 that

$$
\begin{aligned}
\left|\sum_{N<n \leq N+H} \chi(n)\right| & \leq \sum_{a=1}^{v}\left|\sum_{t \in I(a)} \chi_{u}(t)\right| \\
& \ll v u^{(k+1) /\left(4 k^{2}\right)+\varepsilon}\left(\frac{H}{v}\right)^{1-1 / k}
\end{aligned}
$$

providing that $1 \leq H / v \leq u$. However, when $1 \leq H \leq v$, we trivially have

$$
\begin{aligned}
\sum_{N<n \leq N+H} \chi(n) & \ll H \\
& \ll v^{1 / k} H^{1-1 / k} \\
& =v\left(\frac{H}{v}\right)^{1-1 / k} \\
& \ll v u^{(k+1) /\left(4 k^{2}\right)+\varepsilon}\left(\frac{H}{v}\right)^{1-1 / k} .
\end{aligned}
$$

We therefore conclude as follows.

Lemma 2.3 Let $\chi$ be a primitive character to modulus $q$, and let $v$ be the product of those factors $p^{e} \| q$ for which $e \geq 3$. Suppose that $N \geq 1$ and that $1 \leq H \leq q$. Then

$$
\sum_{N<n \leq N+H} \chi(n) \ll_{\varepsilon, k} v^{(3 k-1) /\left(4 k^{2}\right)} q^{(k+1) /\left(4 k^{2}\right)+\varepsilon} H^{1-1 / k}
$$

for any $\varepsilon>0$ and $k \in \mathbb{N}$.

Our next task is to give estimates involving the order of the character $\chi$. We begin by examining the case in which $q$ is a prime power $p^{e}$, and we use the fact that an Abelian group is isomorphic to its character group. The multiplicative group modulo $p^{e}$ has order $\phi\left(p^{e}\right)$ and exponent $n\left(p^{e}\right)$, say, where

$$
n\left(p^{e}\right)= \begin{cases}\phi\left(p^{e}\right), & p \text { odd } \\ 1, & p=2, \quad e=1 \\ 2, & p=2, \quad e=2 \\ 2^{e-2}, & p=2, \quad e \geq 3\end{cases}
$$

Moreover, for $e \geq 2$, there are exactly $p^{-1} \phi\left(p^{e}\right)$ elements whose order divides $p^{-1} n\left(p^{e}\right)$. It follows that there are exactly $\phi\left(p^{e-1}\right)$ characters whose order divides $n\left(p^{e-1}\right)$, except when $p=2$ and $e=3$. However there are $\phi\left(p^{e-1}\right)$ characters to modulus $p^{e-1}$, and the order of these divides $n\left(p^{e-1}\right)$. It follows that the order of a primitive character cannot divide $n\left(p^{e-1}\right)$ when $e \geq 2$, except for $p=2$ and $e=3$. Thus, if $\chi$ is a primitive character of order $l$ and modulus $p^{e}$, we have $p^{e-1} \mid l$ if $p$ is odd and $2^{e-2} \mid l$ for $p=2$. 
We now use Lemma 2.2 to factorize a primitive character modulo $q$ into characters $\chi_{u}$, where $u=p^{e}$, for each $p^{e} \mid q$. If $\chi$ has order dividing $l$, so will $\chi_{u}$, and we conclude that $p^{e-1} \mid 2 l$ whenever $e \geq 2$. Thus, if $v$ is as in Lemma 2.3 we will have $v \mid(2 l)^{2}$. From Lemma 2.3 we then deduce the following.

Lemma 2.4 Let $\chi$ be a primitive character to modulus $q$, and let $l$ be the order of $\chi$. Suppose that $N \geq 1$ and $1 \leq H \leq q$. Then

$$
\sum_{N<n \leq N+H} \chi(n) \ll_{\varepsilon, k} l^{3 / 2 k} q^{(k+1) /\left(4 k^{2}\right)+\varepsilon} H^{1-1 / k}
$$

for any $\varepsilon>0$ and any $k \in \mathbb{N}$. In particular (2.1) holds for all $k \in \mathbb{N}$, providing that the order of $\chi$ is at most $\mathcal{L}$, say.

We turn our attention now to estimates for L-functions. Suppose that $\chi$ is a non-principal primitive character modulo $q$ and that

$$
1-\frac{1}{k} \leq \sigma \leq 1+\frac{\log \mathcal{L}}{\mathcal{L}}
$$

for some integer $k \geq 2$. The Pólya-Vinogradov inequality

$$
\sum_{n \leq N} \chi(n) \ll q^{\frac{1}{2}} \mathcal{L}
$$

yields

$$
\sum_{n>q} \frac{\chi(n)}{n^{s}} \ll q^{\frac{1}{2}-\sigma+\varepsilon}(1+|t|)
$$

by partial summation, if $s=\sigma+i t$. Similarly, for $N \leq q$, Lemmas 2.1 and 2.4 yield

$$
\sum_{n \leq N} \chi(n) \ll q^{(k+1) /\left(4 k^{2}\right)+\varepsilon} N^{1-1 / k} \quad(N \leq q)
$$

for appropriate $k$, so that partial summation produces

$$
\sum_{q_{1}<n \leq q} \frac{\chi(n)}{n^{s}} \ll q_{1}^{1-1 / k-\sigma} q^{(k+1) /\left(4 k^{2}\right)+\varepsilon}(1+|t|)
$$

for $1 \leq q_{1} \leq q$. Finally we have the trivial bound

$$
\sum_{1 \leq n \leq q_{1}} \frac{\chi(n)}{n^{s}} \ll q_{1}^{1-\sigma} q^{\varepsilon} .
$$

We now choose $q_{1}=q^{(k+1) /(4 k)}$, and combine the bounds (2.2),(2.3) and (2.4) to obtain

$$
L(s, \chi) \ll q^{(1-\sigma)(1 / 4+1 / 4 k)+\varepsilon}(1+|t|) .
$$


We may remove the condition that $\chi$ should be primitive via the observation that, if $\chi$ is induced by $\chi^{*}$, then

$$
L(s, \chi) \ll q^{\varepsilon} L\left(s, \chi^{*}\right) \quad\left(\sigma \geq \frac{1}{2}\right) .
$$

In the case in which only $k=2$ and 3 are permissible we shall in fact use $k=3$, so that

$$
\frac{1}{4}+\frac{1}{4 k}=\frac{1}{3} \leq \frac{1}{3}\left(1+k_{0}^{-1}\right) \quad k_{0} \in \mathbb{N} .
$$

We may then conclude as follows.

Lemma 2.5 Let $\chi$ be a non-principal character to modulus $q$, and define $\phi=$ $\phi(\chi)=\frac{1}{4}$ if $q$ is cube-free, or if the order of $\chi$ is at most $\mathcal{L}$; and let $\phi=\frac{1}{3}$ otherwise. Then for any positive integer $k \geq 3$, and any $\varepsilon>0$, we have

$$
L(\sigma+i t, \chi) \ll_{\varepsilon, k} q^{\phi(1-\sigma)\left(1+k^{-1}\right)+\varepsilon}(1+|t|)
$$

uniformly for

$$
1-\frac{1}{k} \leq \sigma \leq 1+\frac{\log \mathcal{L}}{\mathcal{L}}
$$




\section{$3 \quad \frac{L^{\prime}}{L}(s, \chi)$ and the Zeros}

The usual starting point for investigations into $L^{\prime} / L(s, \chi)$ is the partial fraction decomposition

$$
\frac{L^{\prime}}{L}(s, \chi)=\sum_{\rho}\left(\frac{1}{s-\rho}+\frac{1}{\rho}\right)-\frac{1}{2} \log \frac{q}{\pi}-\frac{1}{2} \frac{\Gamma^{\prime}}{\Gamma}\left(\frac{1}{2}(s+a)\right)+B(\chi),
$$

where $\chi$ is a primitive character $(\bmod q),(q>1), \rho$ runs over the non-trivial zeros of $L(s, \chi), a$ is 1 or 0 according as $\chi$ is even or odd, and $B(\chi)$ is a constant depending only on $\chi$. The above formula may be found in Davenport [8; Chapter 12, (17)] for example. If we take real parts, and use the fact that

$$
\Re B(\chi)=-\sum \Re\left(\frac{1}{\rho}\right),
$$

we obtain

$$
\Re \frac{L^{\prime}}{L}(s, \chi)=\sum_{\rho} \Re \frac{1}{s-\rho}-\frac{1}{2} \log q+O(\log (2+|t|)),
$$

for $\frac{1}{2} \leq \sigma \leq 2$, say. If $\chi$ is now non-primitive, but is induced by the primitive character $\chi^{*}\left(\bmod q^{*}\right)$, then

$$
\begin{aligned}
\left|\frac{L^{\prime}}{L}(s, \chi)-\frac{L^{\prime}}{L}\left(s, \chi^{*}\right)\right| & \leq \sum_{p \mid q, p \nmid q^{*}}\left(p^{-\sigma}+p^{-2 \sigma}+\cdots\right) \log p \\
& \leq 2 \sum_{p \mid q / q^{*}} p^{-\sigma} \log p+O(1) \\
& \leq \frac{1}{2} \log q / q^{*}+O(1)
\end{aligned}
$$

for $\sigma \geq \frac{1}{2}$. One therefore has

$$
-\Re \frac{L^{\prime}}{L}(s, \chi) \leq-\sum_{\rho} \Re \frac{1}{s-\rho}+\frac{1}{2} \mathcal{L}+O(\log \log q)
$$

for $\frac{1}{2} \leq \sigma \leq 2,|t| \leq \mathcal{L}$, for any non-principal character, primitive or not.

The term $\frac{1}{2} \mathcal{L}$ in $(3.2)$ plays a key rôle in estimating the width of the zero-free region of $L(s, \chi)$. It is important therefore to see whether one may reduce the constant $\frac{1}{2}$. In order to do this we must discard some of the zeros, since (3.2) is "almost an equality". A device of Stechkin [34] has, until now been the most successful in this context. One replaces $\frac{1}{2} \mathcal{L}$ by

$$
\frac{\sqrt{5}-1}{2 \sqrt{5}} \mathcal{L} \doteq(0.2764) \mathcal{L}
$$


and takes only a finite number of zeros in the sum (3.2). Stechkin's method makes no use of Burgess's bounds, and indeed it is hard to see how they could be incorporated into the argument. We shall therefore give a quite different approach, which reduces the constant 0.2764 to $\frac{\phi}{2}=\frac{1}{6}$ or $\frac{1}{8}$. Even with the trivial estimate $\phi=\frac{1}{2}$ one would reduce 0.2764 to 0.25 by our method.

Lemma 3.1 Let $\chi$ be a non-principal character modulo $q$ and let $\phi$ be as in Lemma 2.5. Then for any $\varepsilon>0$ there exists a $\delta=\delta(\varepsilon)>0$ such that

$$
-\Re \frac{L^{\prime}}{L}(s, \chi) \leq-\sum_{|1+i t-\rho| \leq \delta} \Re \frac{1}{s-\rho}+\left(\frac{\phi}{2}+\varepsilon\right) \mathcal{L}
$$

uniformly for

$$
1+\frac{1}{\mathcal{L} \log \mathcal{L}} \leq \sigma \leq 1+\frac{\log \mathcal{L}}{\mathcal{L}}
$$

and $|t| \leq \mathcal{L}$, providing that $q$ is sufficiently large.

In proving the above we shall apply the following result.

Lemma 3.2 Let $f(z)$ be holomorphic for $|z-a| \leq R$, and non-vanishing both at $z=a$ and on the circle $|z-a|=R$. Let $z_{k}=a+r_{k} \exp \left(i \theta_{k}\right)$ be the zeros of $f(z)$ in the disc, and let $z_{k}$ have multiplicity $n_{k}$. Then

$$
\Re \frac{f^{\prime}}{f}(a)=-\sum n_{k}\left(r_{k}^{-1}-\frac{r_{k}}{R^{2}}\right) \cos \theta_{k}+\frac{1}{\pi R} \int_{0}^{2 \pi}(\cos \theta) \log \left|f\left(a+R e^{i \theta}\right)\right| d \theta .
$$

This formula is related to the well-known theorem of Jensen, and to a less well-known one of Carleman (see Titchmarsh [36; §3.71]). An estimate using terms $\left(r^{-1}-r R^{-2}\right) \cos \theta$ was used by Heilbronn [15; Lemma 11] in connection with zero-free regions, and Miech [27] and Jutila [19] followed the same method. However Heilbronn's estimate is weaker by a factor 4 than what one deduces from Lemma 3.2.

In order to establish Lemma 3.2 we normalize to the case $a=0$, and consider the contour integral

$$
I=\frac{1}{2 \pi i} \oint_{|z|=R}\left(z^{-1}-z R^{-2}\right) \frac{f^{\prime}(z)}{f(z)} d z .
$$

This may be evaluated as

$$
\frac{f^{\prime}}{f}(0)+\sum n_{k}\left(z_{k}^{-1}-z_{k} R^{-2}\right)
$$

by Cauchy's residue theorem. Alternatively, we may integrate by parts to obtain

$$
I=\frac{1}{2 \pi i}\left[\left(z^{-1}-z R^{-2}\right) \log f(z)\right]_{e^{2 \pi i(0+)} R}^{2 \pi i(1-)}+
$$




$$
\begin{array}{r}
+\frac{1}{2 \pi i} \oint\left(z^{-2}+R^{-2}\right) \log f(z) d z \\
=0+\frac{1}{\pi R} \int_{0}^{2 \pi}(\cos \theta) \log f\left(a+R e^{i \theta}\right) d \theta .
\end{array}
$$

The lemma now follows on comparing the real parts of (3.3) and (3.4).

To deduce Lemma 3.1 from Lemma 3.2 we take $f(s)=L(s, \chi)$ and $a=s_{0}=$ $\sigma_{0}+i t_{0}$ (where $s_{0}$ becomes $s$ in Lemma 3.1), and we use a radius $R \leq 1$ to be specified in due course. We require a lower bound for

$$
\int_{0}^{2 \pi}(\cos \theta) \log \left|L\left(s_{0}+R e^{i \theta}, \chi\right)\right| d \theta=J,
$$

say. For $0 \leq \theta \leq \pi / 2$ and $3 \pi / 2 \leq \theta \leq 2 \pi$ we trivially have

$$
\left|\log L\left(s_{0}+R e^{i \theta}, \chi\right)\right| \leq \log \zeta\left(\sigma_{0}+R \cos \theta\right) \leq \log \zeta\left(\sigma_{0}\right) \ll \log \mathcal{L} .
$$

The corresponding contribution to $J$ is therefore $O(\log \mathcal{L})$. If we assume that $R \leq 1 / k$ then in the remaining range $\pi / 2 \leq \theta \leq 3 \pi / 2$ we have

$$
1-\frac{1}{k} \leq \sigma_{0}-\frac{1}{k} \leq \sigma_{0}+R \cos \theta \leq \sigma_{0} \leq 1+\frac{\log \mathcal{L}}{\mathcal{L}} .
$$

We may therefore apply Lemma 2.5 to give

$$
\begin{aligned}
\log \left|L\left(s_{0}+R e^{i \theta}, \chi\right)\right| & \leq\left\{\phi\left(1+\frac{1}{k}\right)\left(1-\sigma_{0}-R \cos \theta\right)+2 \varepsilon\right\} \mathcal{L} \\
& \leq\left\{-\phi\left(1+\frac{1}{k}\right) R \cos \theta+2 \varepsilon\right\} \mathcal{L}
\end{aligned}
$$

for large enough $q$. The contribution to $J$ from such $\theta$ is then

$$
\begin{aligned}
\int_{\pi / 2}^{3 \pi / 2}(\cos \theta) \log \mid L\left(s_{0}\right. & \left.+R e^{i \theta}, \chi\right) \mid d \theta \\
& \geq \mathcal{L} \int_{\pi / 2}^{3 \pi / 2}\left\{-\phi\left(1+\frac{1}{k}\right) R \cos ^{2} \theta+2 \varepsilon \cos \theta\right\} d \theta \\
& =\mathcal{L}\left\{-\frac{\pi R}{2} \phi\left(1+\frac{1}{k}\right)-4 \varepsilon\right\}
\end{aligned}
$$

Notice that the inequality sign becomes reversed in passing from (3.5) to (3.6), since $\cos \theta \leq 0$.

It now follows that

$$
J \geq-\mathcal{L}\left\{\frac{\pi R}{2} \phi\left(1+\frac{1}{k}\right)+5 \varepsilon\right\}
$$


for large enough $q$, and Lemma 3.2 yields

$$
-\Re \frac{L^{\prime}}{L}\left(s_{0}, \chi\right) \leq-\sum_{\left|s_{0}-\rho\right| \leq R} \Re\left(\frac{1}{s_{0}-\rho}-\frac{s_{0}-\rho}{R^{2}}\right)+\mathcal{L}\left\{\frac{1}{2} \phi\left(1+\frac{1}{k}\right)+\frac{5 \varepsilon}{\pi R}\right\} .
$$

We choose $\delta \leq R-\mathcal{L}^{-1} \log \mathcal{L}$, so that the disc $\left|s_{0}-\rho\right| \leq R$ includes $\left|1+i t_{0}-\rho\right| \leq \delta$. We may discard zeros from (3.7) which are not in the smaller disc, since

$$
\Re\left(\frac{1}{s_{0}-\rho}-\frac{s_{0}-\rho}{R^{2}}\right)=\left(\sigma_{0}-\beta\right)\left(\frac{1}{\left|s_{0}-\rho\right|^{2}}-\frac{1}{R^{2}}\right) \geq 0 .
$$

Moreover there are at most $c_{0} \mathcal{L}$ zeros in the sum (for a suitable constant $c_{0}$ ) and

$$
\Re \frac{s_{0}-\rho}{R^{2}}=\frac{\sigma_{0}-\beta}{R^{2}} \leq R^{-2}\left(\frac{\log \mathcal{L}}{\mathcal{L}}+\delta\right)
$$

whenever $\left|1+i t_{0}-\rho\right| \leq \delta$. We therefore see that

$$
\begin{aligned}
-\Re \frac{L^{\prime}}{L}\left(s_{0}, \chi\right) & \leq-\sum_{\left|1+i t_{0}-\rho\right| \leq \delta} \Re\left(\frac{1}{s_{0}-\rho}-\frac{s_{0}-\rho}{R^{2}}\right)+\mathcal{L}\left\{\frac{\phi}{2}\left(1+\frac{1}{k}\right)+\frac{5 \varepsilon}{\pi R}\right\} \\
\leq & -\sum_{\left|1+i t_{0}-\rho\right| \leq \delta} \Re \frac{1}{s_{0}-\rho}+\mathcal{L}\left\{\frac{\phi}{2}\left(1+\frac{1}{k}\right)+\frac{5 \varepsilon}{\pi R}\right\}+ \\
& \leq-\sum_{\left|1+i t_{0}-\rho\right| \leq \delta} \Re \frac{1}{s_{0}-\rho}+\mathcal{L}\left\{\frac{\phi}{2}\left(1+\frac{1}{k}\right)+\frac{6 \varepsilon}{\pi R}+\frac{c_{0} \delta}{R^{2}}\right\}
\end{aligned}
$$

for large enough $q$. It remains to choose the constants $\varepsilon, k, R$, and $\delta$ so as to ensure that $0<R \leq 1 / k, \delta<R$ and

$$
\frac{\phi}{2 k}+\frac{6 \varepsilon}{\pi R}+\frac{c_{0} \delta}{R^{2}} \leq \varepsilon_{0}
$$

say, where $\varepsilon_{0} \in(0,1)$ will become the number $\varepsilon$ appearing in Lemma 3.1. To do this we merely select $k=3+\left[3 \phi / 2 \varepsilon_{0}\right], R=1 / k, \varepsilon=\pi \varepsilon_{0} / 18 k$, and

$$
\delta=\min \left(\frac{1}{2 k}, \frac{\varepsilon_{0}}{3 c_{0} k^{2}}\right) \text {. }
$$

This completes the proof of the lemma.

We conclude by remarking that Lemma 3.2 may be used to obtain zero-free regions for the Riemann Zeta-function, when one has a bound for its order of magnitude in the critical strip. In the past this has often been done via two function theoretic lemmas of Landau (see Titchmarsh [37; §§3.9 and 3.10] for example). Now a single result suffices for the same purpose. 


\section{Zero-Free Regions and Derivatives of $\frac{L^{\prime}}{L}(s, \chi)$}

In order to motivate the next two sections we begin by reviewing the standard procedure for producing a zero-free region for $L(s, \chi)$. For simplicity we describe the situation when $\chi$ is non-real. Let $\rho_{0}=\beta_{0}+i \gamma_{0}$ be a non-trivial zero of $L(s, \chi)$, and let $\sigma \in(1,2]$ be a parameter to be specified later. Since $\sigma>1 \geq \beta$ one has

$$
\Re \frac{1}{s-\rho}=\frac{\sigma-\beta}{(\sigma-\beta)^{2}+(t-\gamma)^{2}} \geq 0
$$

for any zero $\rho=\beta+i \gamma$. If one uses this in (3.2) for all zeros $\rho \neq \rho_{0}$, one finds

$$
-\Re \frac{L^{\prime}}{L}\left(\sigma+i \gamma_{0}, \chi\right) \leq-\frac{1}{\sigma-\beta_{0}}+\frac{1}{2} \mathcal{L}+O(\log \mathcal{L}) .
$$

Similarly, if one discards all the zeros one has

$$
-\Re \frac{L^{\prime}}{L}\left(\sigma+2 i \gamma_{0}, \chi^{2}\right) \leq \frac{1}{2} \mathcal{L}+O(\log \mathcal{L})
$$

since $\chi^{2}$ is non-principal. Finally, for the Riemann Zeta-function one has

$$
-\frac{\zeta^{\prime}}{\zeta}(\sigma)=\frac{1}{\sigma-1}+O(1)
$$

At this point one appeals to the inequality

$$
3+4 \cos \theta+\cos 2 \theta=2(1+\cos \theta)^{2} \geq 0
$$

whence

$$
3+4 \Re\left\{\frac{\chi(n)}{n^{i \gamma_{0}}}\right\}+\Re\left\{\frac{\chi^{2}(n)}{n^{2 i \gamma_{0}}}\right\} \geq 0
$$

and

$$
\begin{aligned}
-3 \frac{\zeta^{\prime}}{\zeta}(\sigma)-4 \Re\left\{\frac{L^{\prime}}{L}\left(\sigma+i \gamma_{0}, \chi\right)\right\} & -\Re\left\{\frac{L^{\prime}}{L}\left(\sigma+2 i \gamma_{0}, \chi^{2}\right)\right\} \\
& =\sum_{n=1}^{\infty} \frac{\Lambda(n)}{n^{\sigma}}\left(3+4 \Re\left\{\frac{\chi(n)}{n^{i \gamma_{0}}}\right\}+\Re\left\{\frac{\chi^{2}(n)}{n^{2 i \gamma_{0}}}\right\}\right) \\
& \geq 0 .
\end{aligned}
$$

It follows that

$$
\frac{3}{\sigma-1}-\frac{4}{\sigma-\beta_{0}}+\frac{5}{2} \mathcal{L}+O(\log \mathcal{L}) \geq 0 .
$$

One now makes the optimal choice of $\sigma$, namely

$$
\sigma=1+(3+2 \sqrt{3})\left(1-\beta_{0}\right)
$$


whence

$$
-\frac{1}{(7+4 \sqrt{3})\left(1-\beta_{0}\right)}+\frac{5}{2} \mathcal{L}+O(\log \mathcal{L}) \geq 0
$$

and

$$
\beta_{0} \leq 1-\frac{1}{(7+4 \sqrt{3})\left(\frac{5}{2} \mathcal{L}+O(\log \mathcal{L})\right)} \leq 1-\frac{1}{(34.82 \ldots) \mathcal{L}} .
$$

Of course, if $\sigma$, given by (4.5), falls outside the range $1<\sigma \leq 2$, then (4.6) still holds.

One may improve the above result by using different inequalities of the form (4.2), thereby changing the coefficients 3,4 and $\frac{5}{2}$ in (4.4). Moreover it is clear that if one can reduce the term $\frac{1}{2} \mathcal{L}$ in (3.2) one will get a corresponding improvement in (4.6). Thus Lemma 3.1 immediately leads to

$$
\beta \leq 1-\frac{1}{c \mathcal{L}}
$$

with

$$
c=(7+4 \sqrt{3}) \frac{5 \phi}{2}+o(1)=\left\{\begin{array}{cc}
11.60 \ldots, & \phi=\frac{1}{3} \\
8.70 \ldots, & \phi=\frac{1}{4} .
\end{array}\right.
$$

The idea we wish to describe in this section starts with the observation that one may differentiate $(3.1) k$ times to obtain

$$
\frac{d^{k}}{d s^{k}} \frac{L^{\prime}}{L}(s, \chi)=(-1)^{k} k ! \sum_{\rho} \frac{1}{(s-\rho)^{k+1}}-\frac{1}{2} \frac{d^{k}}{d s^{k}} \frac{\Gamma^{\prime}}{\Gamma}\left(\frac{1}{2}(s+a)\right),
$$

whence

$$
\Re\left(\frac{(-1)^{k}}{k !} \frac{d^{k}}{d s^{k}} \frac{L^{\prime}}{L}(s, \chi)\right)=\sum_{\rho} \Re \frac{1}{(s-\rho)^{k+1}}+O(1) .
$$

Here there is no term in $\mathcal{L}$. Unfortunately it is not possible to use this formula as a direct substitute for $(3.2)$ since $\Re(s-\rho)^{-k-1}$ is not one signed. However one does have, for example

$$
\Re\left(\frac{1}{s-\rho}+(\sigma-1) \frac{1}{(s-\rho)^{2}}\right)=\frac{(2 \sigma-\beta-1)(\sigma-\beta)^{2}+(1-\beta)(t-\gamma)^{2}}{\left[(\sigma-\beta)^{2}+(t-\gamma)^{2}\right]^{2}} \geq 0 .
$$

Hence, if we write temporarily

$$
\begin{aligned}
f(s, \chi) & =\Re\left\{-\frac{L^{\prime}}{L}(s, \chi)+(\sigma-1)\left(\frac{L^{\prime}}{L}\right)^{\prime}(s, \chi)\right\} \\
& =\sum_{n=1}^{\infty} \frac{\Lambda(n)}{n^{\sigma}}\{1+(\sigma-1) \log n\} \Re \frac{\chi(n)}{n^{i t}}
\end{aligned}
$$


we find

$$
\begin{aligned}
f\left(\sigma+i \gamma_{0}, \chi\right) & \leq-\frac{1}{\sigma-\beta_{0}}-\frac{\sigma-1}{\left(\sigma-\beta_{0}\right)^{2}}+\frac{1}{2} \mathcal{L}+O(\log \mathcal{L}), \\
f\left(\sigma+2 i \gamma_{0}, \chi^{2}\right) & \leq \frac{1}{2} \mathcal{L}+O(\log \mathcal{L})
\end{aligned}
$$

and

$$
f(\sigma, 1)=\frac{1}{\sigma-1}+\frac{\sigma-1}{(\sigma-1)^{2}}+O(1) .
$$

Moreover, since $1+(\sigma-1) \log n \geq 0$, we have

$$
3 f(\sigma, 1)+4 f\left(\sigma+i \gamma_{0}, \chi\right)+f\left(\sigma+2 i \gamma_{0}, \chi^{2}\right) \geq 0,
$$

by $(4.3)$, so that

$$
\frac{6}{\sigma-1}-4\left(\frac{1}{\sigma-\beta_{0}}+\frac{\sigma-1}{\left(\sigma-\beta_{0}\right)^{2}}\right)+\frac{5}{2} \mathcal{L}+O(\log \mathcal{L}) \geq 0 .
$$

Now the optimal choice is

$$
\sigma=1+\left\{3+(36)^{1 / 3}+(48)^{1 / 3}\right\}\left(1-\beta_{0}\right),
$$

whence

$$
\beta_{0} \leq 1-\frac{1}{\frac{1}{14}\left(50+27.6^{1 / 3}+15.6^{2 / 3}\right)\left(\frac{5}{2} \mathcal{L}+O(\log \mathcal{L})\right)} \leq 1-\frac{1}{(26.53 \ldots) \mathcal{L}} .
$$

If we use Lemma 3.1 we find instead that

$$
\beta_{0} \leq 1-\frac{1}{c \mathcal{L}}
$$

with

$$
c= \begin{cases}8.84 \ldots, & \phi=\frac{1}{3} \\ 6.63 \ldots, & \phi=\frac{1}{4}\end{cases}
$$

This improves the earlier result by about one third.

One may extend this technique further by taking more complicated combinations of derivatives. In place of the result (4.9) we shall use inequalities of the form

$$
\Re p\left(\frac{1}{z}\right) \geq 0 \quad \text { for } \quad \Re z \geq 1
$$

where

$$
p(X)=\sum_{k=1}^{d} a_{k} X^{k}
$$


is a polynomial with real, non-negative coefficients. One then has

$$
\Re p\left(\frac{\sigma-1}{s-\rho}\right) \geq 0, \quad(\sigma>1) .
$$

We may therefore follow through the previous argument, taking

$$
\begin{aligned}
f(s, \chi) & =\frac{1}{\sigma-1} \Re\left\{\sum_{k=1}^{d} a_{k} \frac{(-1)^{k}}{(k-1) !}(\sigma-1)^{k} \frac{d^{k-1}}{d s^{k-1}}\left(\frac{L^{\prime}}{L}(s, \chi)\right)\right\} \\
& =\sum_{n=1}^{\infty} \frac{\Lambda(n)}{n^{\sigma}}\left\{\sum_{k=1}^{d} a_{k} \frac{((\sigma-1) \log n)^{k-1}}{(k-1) !}\right\} \Re \frac{\chi(n)}{n^{i t}},
\end{aligned}
$$

and obtaining

$$
\frac{3 p(1)}{\sigma-1}-\frac{4 p\left(\frac{\sigma-1}{\sigma-\beta_{0}}\right)}{\sigma-1}+\frac{5}{2} a_{1} \mathcal{L}+O(\log \mathcal{L}) \geq 0 .
$$

In order to establish inequalities of the type (4.10) the following corollary to the maximum modulus principle is useful.

Lemma 4.1 Let $F_{1}(z)$ and $F_{2}(z)$ be holomorphic on $\mathcal{H}=\{z \in \mathbb{C}: \Re z \geq 0\}$, and suppose that $\Re F_{1}(z) \geq\left|F_{2}(z)\right|$ for $\Re z=0$. Suppose further that $F_{1}$ and $F_{2}$ tend uniformly to 0 on $\mathcal{H}$ as $|z| \rightarrow \infty$. Then $\Re F_{1}(z) \geq\left|F_{2}(z)\right|$ throughout $\mathcal{H}$.

We prove this by contradiction. Suppose that $\Re F_{1}\left(z_{0}\right)<\left|F_{2}\left(z_{0}\right)\right|$ for some $z_{0} \in \mathcal{H}$, and set

$$
\delta=\frac{1}{3}\left(\left|F_{2}\left(z_{0}\right)\right|-\Re F_{1}\left(z_{0}\right)\right)>0 .
$$

Then

$$
\left|\Re F_{1}(z)\right|,\left|F_{2}(z)\right| \leq \delta
$$

on $|z|=R$, if $R>\left|z_{0}\right|$ is chosen large enough. Let

$$
F_{2}\left(z_{0}\right)=e^{i \theta}\left|F_{2}\left(z_{0}\right)\right|
$$

and define

$$
F_{3}(z)=F_{1}(z)-e^{-i \theta} F_{2}(z)+2 \delta .
$$

On the boundary of the region

$$
\mathcal{H}_{R}=\{z \in \mathcal{H} ;|z| \leq R\}
$$

we have $\Re F_{3} \geq 0$, so that $\Re F_{3} \geq 0$ throughout $\mathcal{H}_{R}$. It follows that

$$
0 \leq \Re F_{3}\left(z_{0}\right)=\Re F_{1}\left(z_{0}\right)-\left|F_{2}\left(z_{0}\right)\right|+2 \delta=-\delta .
$$

This contradiction proves the lemma. 
In our case we take $F_{1}(z)=p\left(\frac{1}{1+z}\right)$ and $F_{2}(z)=0$. It then suffices to check that

$$
\Re p\left(\frac{1}{1+i y}\right) \geq 0 .
$$

This is readily done, for instance when

$$
p(X)=X, \quad X+X^{2}, \quad X+X^{2}+\frac{2}{3} X^{3}, \quad \text { or } \quad X+X^{2}+\frac{4}{5} X^{3}+\frac{2}{5} X^{4} .
$$

The first two of these correspond to the inequalities (4.1) and (4.9). Rather than search for polynomials which yield good zero-free regions we shall, in the next section, generalize our method further, to allow non-polynomial functions. We will then be able to find the optimal function in certain problems by an appeal to the calculus of variations. It turns out that these optimal functions are not polynomials. None the less the procedure of this section is still of value in as much as the polynomials (4.12) are very convenient for calculations. 


\section{An "Explicit Formula"}

In this section we shall prove a formula relating the sum

$$
\sum=\sum_{n=1}^{\infty} \Lambda(n) \frac{\chi(n)}{n^{s}} f\left(\mathcal{L}^{-1} \log n\right)
$$

to a sum over zeros. This will be related to the well known formula for $\psi(x)$, as well as to the partial fraction decompositions (3.1) and (4.7). We shall suppose that $f$ satisfies the condition below.

Condition 1 Let $f$ be a continuous function from $[0, \infty)$ to $\mathbb{R}$, supported in $\left[0, x_{0}\right)$, and let $f$ be twice differentiable on $\left(0, x_{0}\right)$, with $f^{\prime \prime}$ being continuous and bounded by $B$.

This condition can be modified substantially, but will suffice for our purposes. We note that

$$
\left|f^{\prime}\left(t_{0}\right)\right|=\left|\frac{1}{x_{0}}\left(f\left(x_{0}\right)-f(0)\right)\right|=\frac{|f(0)|}{x_{0}}
$$

for some $t_{0} \in\left(0, x_{0}\right)$, whence

$$
\begin{aligned}
\left|f^{\prime}(t)\right| \leq\left|f^{\prime}(t)-f^{\prime}\left(t_{0}\right)\right|+\left|f^{\prime}\left(t_{0}\right)\right| & \leq B\left|t-t_{0}\right|+x_{0}^{-1}|f(0)| \\
& \leq B x_{0}+\frac{|f(0)|}{x_{0}}
\end{aligned}
$$

for all $t \in\left(0, x_{0}\right)$. Similarly we find

$$
|f(t)|=\left|f(t)-f\left(x_{0}\right)\right| \leq\left|t-x_{0}\right|\left\{B x_{0}+\frac{|f(0)|}{x_{0}}\right\} \leq B x_{0}^{2}+|f(0)|
$$

for all $t \in\left(0, x_{0}\right)$.

We write

$$
F(z)=\int_{0}^{\infty} e^{-z t} f(t) d t
$$

for the Laplace transform of $f$. Since $f$ has compact support, $F(z)$ is entire. In the region $\Re z>0$ we have

$$
\begin{aligned}
F(z) & =\int_{0}^{x_{0}} e^{-z t} f(t) d t \\
& =\frac{1}{z} f(0)+\frac{1}{z} \int_{0}^{x_{0}} e^{-z t} f^{\prime}(t) d t \\
& =\frac{1}{z} f(0)+F_{0}(z),
\end{aligned}
$$

say. Here

$$
F_{0}(z)=z^{-2}\left[f^{\prime}(0+)-f^{\prime}\left(x_{0}-\right) e^{-z x_{0}}\right]+z^{-2} \int_{0}^{x_{0}} e^{-z t} f^{\prime \prime}(t) d t
$$


whence

$$
\left|F_{0}(z)\right| \leq|z|^{-2}\left(3 B x_{0}+2 \frac{|f(0)|}{x_{0}}\right)=|z|^{-2} A(f)
$$

say.

Now let $\sigma \geq 1+2 \mathcal{L}^{-1}$, and let $\chi$ be a primitive character to modulus $q_{1}$, where $q_{1} \mid q, q_{1} \neq 1$. We put $\alpha=1+\mathcal{L}^{-1}$, and consider

$$
I=\frac{1}{2 \pi i} \int_{\alpha-i \infty}^{\alpha+i \infty}\left(-\frac{L^{\prime}}{L}(w, \chi)\right) F_{0}((s-w) \mathcal{L}) d w .
$$

This may be calculated by termwise integration. We have

$$
\begin{aligned}
\frac{1}{2 \pi i} \int_{\alpha-i \infty}^{\alpha+i \infty} n^{-w} F_{0} & ((s-w) \mathcal{L}) d w \\
& =\frac{n^{-s}}{2 \pi i} \int_{\sigma-\alpha-i \infty}^{\sigma-\alpha+i \infty} n^{u} F_{0}(u \mathcal{L}) d u \\
& =\mathcal{L}^{-1} \frac{n^{-s}}{2 \pi i} \lim _{T \rightarrow \infty} \int_{\sigma-\alpha-i T}^{\sigma-\alpha+i T} \frac{n^{u}}{u} \int_{0}^{x_{0}} e^{-u \mathcal{L} t} f^{\prime}(t) d t d u \\
& =\mathcal{L}^{-1} \frac{n^{-s}}{2 \pi i} \lim _{T \rightarrow \infty} \int_{0}^{x_{0}} f^{\prime}(t) \int_{\sigma-\alpha-i T}^{\sigma-\alpha+i T} \frac{n^{u} e^{-u \mathcal{L} t}}{u} d u d t
\end{aligned}
$$

by Fubini's Theorem. However

$$
\int_{\sigma-\alpha-i T}^{\sigma-\alpha+i T} \frac{\left(n e^{-\mathcal{L} t}\right)^{u}}{u} d u \ll_{\sigma, n, q} 1
$$

uniformly for $T \geq 0$ and $0 \leq t \leq x_{0}$, and

$$
\lim _{T \rightarrow \infty} \frac{1}{2 \pi i} \int_{\sigma-\alpha-i T}^{\sigma-\alpha+i T} \frac{\left(n e^{-\mathcal{L} t}\right)^{u}}{u} d u= \begin{cases}1, & n>e^{\mathcal{L} t} \\ 0, & n<e^{\mathcal{L} t}\end{cases}
$$

Hence Lebesgue's Dominated Convergence Theorem yields

$$
\begin{aligned}
\frac{1}{2 \pi i} \int_{\alpha-i \infty}^{\alpha+i \infty} n^{-w} F_{0}((s-w) \mathcal{L}) d w & =\mathcal{L}^{-1} n^{-s} \int_{0}^{\min \left(x_{0}, \mathcal{L}^{-1} \log n\right)} f^{\prime}(t) d t \\
& =\mathcal{L}^{-1} n^{-s}\left\{f\left(\mathcal{L}^{-1} \log n\right)-f(0)\right\} .
\end{aligned}
$$

We therefore obtain

$$
I=\mathcal{L}^{-1} \sum+\mathcal{L}^{-1} \frac{L^{\prime}}{L}(s, \chi) f(0)
$$

We now move the line of integration in (5.5) to $\Re w=-\frac{1}{2}$, giving

$$
I=\frac{1}{2 \pi i} \int_{-\frac{1}{2}-i \infty}^{-\frac{1}{2}+i \infty}\left(-\frac{L^{\prime}}{L}(w, \chi)\right) F_{0}((s-w) \mathcal{L}) d w-\sum_{\rho} F_{0}((s-\rho) \mathcal{L}) .
$$


According to the functional equation for $L(w, \chi)$ we have

$$
\begin{aligned}
\frac{L^{\prime}}{L}(w, \chi) & =-\frac{L^{\prime}}{L}(1-w, \bar{\chi})-\log q_{1}+O(\log (2+|w|)) \\
& =-\log q_{1}+O(\log (2+|w|))
\end{aligned}
$$

for $\Re w=-\frac{1}{2}$. Thus

$$
\begin{aligned}
\frac{1}{2 \pi i} \int_{-\frac{1}{2}-i \infty}^{-\frac{1}{2}+i \infty}\left(-\frac{L^{\prime}}{L}(w, \chi)\right) F_{0}((s-w) \mathcal{L}) d w & \\
= & \frac{\log q_{1}}{2 \pi i} \int_{-\frac{1}{2}-i \infty}^{-\frac{1}{2}+i \infty} F_{0}((s-w) \mathcal{L}) d w \\
& +O\left(\frac{A(f)}{\mathcal{L}^{2}} \int_{-\frac{1}{2}-i \infty}^{-\frac{1}{2}+i \infty} \frac{\log (2+|w|)}{|s-w|^{2}}|d w|\right),
\end{aligned}
$$

by (5.4). The first integral on the right vanishes, as one sees by moving the line of integration to the left and appealing to (5.4). Moreover the error term is $O\left(A(f) \mathcal{L}^{-2} \log (2+|s|)\right)$. It follows that

$$
I=-\sum_{\rho} F_{0}((s-\rho) \mathcal{L})+O\left(A(f) \mathcal{L}^{-2} \log (2+|s|)\right) .
$$

From (5.6) we therefore see that

$$
\begin{aligned}
\sum=-\mathcal{L} \sum_{\rho} F_{0}((s-\rho) \mathcal{L})-\frac{L^{\prime}}{L}(s, \chi) f(0) & \\
& +O\left(A(f) \mathcal{L}^{-1} \log (2+|s|)\right) .
\end{aligned}
$$

In particular when $f(0)=0$ we have

$$
\sum_{n=1}^{\infty} \Lambda(n) \frac{\chi(n)}{n^{s}} f\left(\mathcal{L}^{-1} \log n\right)=-\mathcal{L} \sum_{\rho} F((s-\rho) \mathcal{L})+O\left(A(f) \mathcal{L}^{-1} \log (2+|s|)\right) .
$$

If we write $g(t)=e^{\alpha t} f(t)$ then

$$
n^{-(s-\alpha / \mathcal{L})} f\left(\mathcal{L}^{-1} \log n\right)=n^{-s} g\left(\mathcal{L}^{-1} \log n\right) .
$$

The Laplace transform of $g(t)$ is merely $F(z-\alpha)$. Moreover $g$ will still satisfy Condition 1 with the same $x_{0}$ as before. However, according to (5.1) and (5.2) we have to replace $B$ by

$$
e^{\alpha x_{0}}\left\{B+2 \alpha\left(B x_{0}+\frac{|f(0)|}{x_{0}}\right)+\alpha^{2}\left(B x_{0}^{2}+|f(0)|\right)\right\} .
$$


In view of (5.4) we therefore have

$$
A(g) \ll_{B, x_{0},|f(0)|} \mathcal{L}^{1 / 2},
$$

if $0 \leq \alpha \leq(\log \mathcal{L}) /\left(3 x_{0}\right)$. It follows that

$$
\begin{aligned}
\left.\left.\sum_{n=1}^{\infty} \frac{\chi(n)}{n^{s-\alpha / \mathcal{L}} f\left(\mathcal{L}^{-1} \log n\right)=-\mathcal{L} \sum_{\rho} F((s}-\frac{\alpha}{\mathcal{L}}-\rho\right) \mathcal{L}\right) \\
+O_{B, x_{0}}\left(\mathcal{L}^{-1 / 2} \log (2+|s|)\right)
\end{aligned}
$$

for $f(0)=0, \Re s \geq 1+2 \mathcal{L}^{-1}$. We conclude as follows.

Lemma 5.1 Let $\chi$ be a primitive character modulo $q>1$, and let

$$
\Re s \geq 1-\frac{(\log \mathcal{L})^{1 / 2}}{\mathcal{L}} .
$$

Suppose that $f$ satisfies Condition 1, and that $f(0)=0$. Then

$$
\begin{aligned}
\sum_{n=1}^{\infty} \Lambda(n) \frac{\chi(n)}{n^{s}} f\left(\mathcal{L}^{-1} \log n\right) & \\
& =-\mathcal{L} \sum_{\rho} F((s-\rho) \mathcal{L})+O_{B, x_{0}}\left(\mathcal{L}^{-1 / 2} \log (2+|s|)\right)
\end{aligned}
$$

This is the explicit formula referred to earlier. However for applications we require $f(0) \neq 0$, and this entails using Lemma 3.1. We discard those terms in (5.7) with $|1+i t-\rho| \geq \delta$, with error

$$
\begin{aligned}
\ll \mathcal{L} \sum_{|1+i t-\rho| \geq \delta} \frac{A(f)}{\mathcal{L}^{2}|s-\rho|^{2}} & \ll \delta \quad \mathcal{L}^{-1} A(f) \sum_{\rho} \frac{1}{1+|t-\gamma|^{2}} \\
& \ll A(f) \log (2+|s|) .
\end{aligned}
$$

On taking real parts we therefore have

$$
\begin{gathered}
\Re\left\{\sum\right\}=-\sum_{|1+i t-\rho| \leq \delta}\left\{\mathcal{L} \Re\{F((s-\rho) \mathcal{L})\}-\Re\left(\frac{f(0)}{s-\rho}\right)\right\}-f(0) \Re\left(\frac{L^{\prime}}{L}(s, \chi)\right) \\
+O(A(f) \log (2+|s|)) \\
\leq-\mathcal{L} \sum_{|1+i t-\rho| \leq \delta} \Re\{F((s-\rho) \mathcal{L})\}+f(0)\left(\frac{\phi}{2}+\varepsilon\right) \mathcal{L} \\
+O(A(f) \log (2+|s|))
\end{gathered}
$$

providing that $f(0) \geq 0$. We may now proceed as in the proof of Lemma 5.1 , to extend the range for $\sigma$. The inequality (5.8) then holds for $\sigma \geq 1-\mathcal{L}^{-1}(\log \mathcal{L})^{1 / 2}$, 
with error term $O_{B, x_{0}}\left(\mathcal{L}^{1 / 2}(\log (2+|s|))\right.$. Finally we may replace the sum $\sum$ by that corresponding to the character $(\bmod q)$ induced by $\chi$. The error in so doing is at most

$$
\sum^{\prime} \frac{\Lambda(n)}{n^{\sigma}} f\left(\mathcal{L}^{-1} \log n\right) \ll_{B, x_{0}} \sum^{\prime} 1 \ll_{B, x_{0}} \frac{\mathcal{L}}{\log \mathcal{L}},
$$

where $\sum^{\prime}$ denotes summation over prime powers $n=p^{e} \leq q^{x_{0}}$ with $p \mid q$. We now conclude as follows.

Lemma 5.2 Let $\chi$ be a non-principal character $(\bmod q)$ and let $s=\sigma+i t$ with

$$
|\sigma-1| \leq \frac{(\log \mathcal{L})^{1 / 2}}{\mathcal{L}},|t| \leq \mathcal{L}
$$

Suppose $f$ satisfies Condition 1 and that $f(0) \geq 0$. Then, for any $\varepsilon>0$ there is a corresponding $\delta \in(0,1)$, which may depend on $f$, but not on $\chi, q$ or $s$ such that

$$
\begin{aligned}
& \sum_{n=1}^{\infty} \Re\left(\frac{\chi(n)}{n^{s}}\right) f\left(\mathcal{L}^{-1} \log n\right) \\
& \leq-\mathcal{L} \sum_{|1+i t-\rho| \leq \delta} \Re\{F((s-\rho) \mathcal{L})\}+f(0)\left(\frac{\phi}{2}+\varepsilon\right) \mathcal{L},
\end{aligned}
$$

providing that $q$ is sufficiently large.

It should be remarked that one can adjust $\sigma$ by making changes to $f$ and $F$ as already described. However the given range for $\sigma$ appears to be the most suggestive. We also remark that one may take $\delta=1 / \log \mathcal{L}$, independent of $f$, since this may be done in Lemma 3.1. However the "sufficiently large" condition on $q$ will still depend on $f$.

We conclude this section by establishing the analogous result for the principal character $(\bmod q)$. If we write $\psi(x)=x+R(x)$ then one finds from partial summation that

$$
\begin{aligned}
\sum_{A<n \leq B} \frac{\Lambda(n)}{n^{s}} f\left(\mathcal{L}^{-1} \log n\right)= & \int_{A}^{B} t^{-s} f\left(\mathcal{L}^{-1} \log t\right) d t+O\left(\frac{|R(B)|}{B^{\sigma}}\right) \\
& +O\left(\frac{|R(A)|}{A^{\sigma}}\right)+O\left(\left(|s|+\mathcal{L}^{-1}\right) \int_{A}^{B}|R(t)| t^{-1-\sigma} d t\right) .
\end{aligned}
$$

Since $\sigma \geq 1-\mathcal{L}^{-1}(\log \mathcal{L})^{1 / 2}$ and

$$
R(t) \ll t \exp \left(-(\log t)^{1 / 2}\right)
$$

we have $R(t) t^{-\sigma} \ll \mathcal{L}^{-2}$ for

$$
t \geq A, A=\exp \left(4 \log ^{2} \mathcal{L}\right) .
$$


Thus

$$
\sum_{n>A} \frac{\Lambda(n)}{n^{s}} f\left(\mathcal{L}^{-1} \log n\right)=\int_{A}^{\infty} t^{-s} f\left(\mathcal{L}^{-1} \log t\right) d t+O(1)
$$

on taking $B=\exp \left(\mathcal{L} x_{0}\right)$. On noting that

$$
\sum_{n \leq A} \frac{\Lambda(n)}{n^{s}} f\left(\mathcal{L}^{-1} \log n\right) \ll A^{\mathcal{L}^{-1}(\log \mathcal{L})^{1 / 2}} \log A \ll \log ^{2} \mathcal{L}
$$

and similarly

$$
\int_{1}^{A} t^{-s} f\left(\mathcal{L}^{-1} \log t\right) d t \ll A^{\mathcal{L}^{-1}(\log \mathcal{L})^{1 / 2}} \log A \ll \log ^{2} \mathcal{L}
$$

we deduce that

$$
\begin{aligned}
\sum_{n=1}^{\infty} \frac{\Lambda(n)}{n^{s}} f\left(\mathcal{L}^{-1} \log n\right) & =\int_{1}^{\infty} t^{-s} f\left(\mathcal{L}^{-1} \log t\right) d t+O\left(\log ^{2} \mathcal{L}\right) \\
& =\mathcal{L} F(s-1)+O\left(\log ^{2} \mathcal{L}\right)
\end{aligned}
$$

Finally we observe that

$$
\sum_{\chi_{0}(n) \neq 1} \frac{\Lambda(n)}{n^{s}} f\left(\mathcal{L}^{-1} \log n\right) \ll \omega(q) \ll \frac{\mathcal{L}}{\log \mathcal{L}} .
$$

We therefore conclude as follows.

Lemma 5.3 Let $s=\sigma+i t$ with

$$
|\sigma-1| \leq \frac{(\log \mathcal{L})^{1 / 2}}{\mathcal{L}},|t| \leq \mathcal{L} .
$$

Suppose $f$ satisfies Condition 1. Then

$$
\sum_{n=1}^{\infty} \Lambda(n) \frac{\chi_{0}(n)}{n^{s}} f\left(\mathcal{L}^{-1} \log n\right)=\mathcal{L} F((s-1) \mathcal{L})+O\left(\frac{\mathcal{L}}{\log \mathcal{L}}\right)
$$




\section{Zero-Free Regions - Preliminaries}

We begin by proving the following result.

Lemma 6.1 For all suficiently large $q$ there exists a positive integer $L \leq \frac{1}{10} \mathcal{L}$, depending on $q$, such that none of the functions $L(s, \chi)$ with characters $\chi(\bmod q)$ have zeros in the rectangles

$$
1-\frac{\log \log \mathcal{L}}{3 \mathcal{L}} \leq \sigma \leq 1, L<|t| \leq 10 L
$$

This is an easy consequence of the estimate

$$
\sum_{\chi(\bmod q)} N(\sigma, T, \chi) \ll_{\varepsilon}(q T)^{(2+\varepsilon)(1-\sigma)}\left(\frac{4}{5} \leq \sigma \leq 1, T \geq 1, \varepsilon>0\right)
$$

due to Jutila [21; Theorem 1]. For if none of $L=10^{k}, k=0,1,2, \ldots,\left[\frac{\log \mathcal{L} / 10}{\log 10}\right]$ satisfies the conditions of the lemma, one would have

$$
\sum_{\chi(\bmod q)} N(\sigma, \mathcal{L}, \chi) \geq 1+\left[\frac{\log \mathcal{L} / 10}{\log 10}\right] \gg \log \mathcal{L}, \quad\left(\sigma=1-\frac{\log \log \mathcal{L}}{3 \mathcal{L}}\right) .
$$

However, with this value of $\sigma$, Jutila's bound implies

$$
\begin{aligned}
\sum_{\chi(\bmod q)} N(\sigma, \mathcal{L}, \chi) & \ll_{\varepsilon}(q \mathcal{L})^{(2+\varepsilon)(1-\sigma)} \ll_{\varepsilon} q^{(2+2 \varepsilon)(1-\sigma)} \\
& =(\log \mathcal{L})^{(2+2 \varepsilon) / 3}
\end{aligned}
$$

giving a contradiction for large enough $q$, on choosing $\varepsilon=\frac{1}{3}$.

We proceed to number certain of the characters $\chi(\bmod q)$ and zeros $\rho=\beta+i \gamma$ of $L(s, \chi)$ as follows. Let $\rho_{1}$ be a zero in the rectangle

$$
R=\left\{s: 1-\frac{\log \log \mathcal{L}}{3 \mathcal{L}} \leq \sigma \leq 1,|t| \leq L\right\}
$$

for which $\beta$ is maximal, and let $\chi_{1}$ be a corresponding character. Now eliminate all zeros of $L\left(s, \chi_{1}\right)$ and $L\left(s, \overline{\chi_{1}}\right)$, and choose $\rho_{2}$ to be one of the remaining zeros in $R$, for which $\beta$ is maximal. We take $\chi_{2}$ to be a character for which $L\left(\rho_{2}, \chi_{2}\right)=0$. One continues in this way until there are no further zeros in $R$ to be considered. That is to say, at each stage we eliminate all zeros of $L\left(s, \chi_{i}\right)$ and $L\left(s, \overline{\chi_{i}}\right)$ for $1 \leq i \leq k$, and then choose $\rho_{k+1}$, from the remaining zeros, to have $\beta$ maximal. It follows that, if $\chi \neq \chi_{i}, \overline{\chi_{i}}$ for $1 \leq i<k$, then every zero of $L(s, \chi)$ satisfies

$$
\Re(\rho) \leq \Re\left(\rho_{k}\right) \quad \text { or } \quad|\Im(\rho)| \geq 10 L .
$$

We observe that

$$
\chi_{i} \neq \chi_{j}, \overline{\chi_{j}} \quad \text { for } \quad i \neq j \text {. }
$$


For convenience of notation we shall set

$$
\rho_{k}=\beta_{k}+i \gamma_{k}, \beta_{k}=1-\mathcal{L}^{-1} \lambda_{k}, \gamma_{k}=\mathcal{L}^{-1} \mu_{k} .
$$

To see how this information may be used in practice, let us consider estimates for $\lambda_{2}$. We shall use Lemmas 5.2 and 5.3, and we therefore write

$$
K(s, \chi)=\sum_{n=1}^{\infty} \Lambda(n) \Re\left(\frac{\chi(n)}{n^{s}}\right) f\left(\mathcal{L}^{-1} \log n\right),
$$

for ease of notation. Since $\left|\chi(n) n^{-i \gamma}\right| \leq \chi_{0}(n)$, we have the inequality

$$
\left\{\chi_{0}(n)+\Re\left(\chi_{1}(n) n^{-i \gamma_{1}}\right)\right\}\left\{\chi_{0}(n)+\Re\left(\chi_{2}(n) n^{-i \gamma_{2}}\right)\right\} \geq 0 .
$$

We now impose the following condition on $f$.

Condition 2 The function $f$ is non-negative. Moreover its Laplace transform satisfies

$$
\Re(F(z)) \geq 0 \text { for } \Re(z) \geq 0 .
$$

Since $f$ is non-negative it follows that

$$
\begin{aligned}
& K\left(\beta_{1}, \chi_{0}\right)+K\left(\beta_{1}+i \gamma_{1}, \chi_{1}\right)+K\left(\beta_{1}+i \gamma_{2}, \chi_{2}\right) \\
& \quad+\frac{1}{2} K\left(\beta_{1}+i \gamma_{1}+i \gamma_{2}, \chi_{1} \chi_{2}\right)+\frac{1}{2} K\left(\beta_{1}+i \gamma_{1}-i \gamma_{2}, \chi_{1} \overline{\chi_{2}}\right) \geq 0 .
\end{aligned}
$$

Since $\delta<1 \leq L$ and $\left|\gamma_{i}\right| \leq L$ the condition

$$
\left|1+i \gamma_{1}+i \gamma_{2}-\rho\right| \leq \delta
$$

implies

$$
|\Im(\rho)| \leq 3 L<10 L .
$$

It follows from the choice of $\rho_{1}$ that all relevant zeros of $L\left(s, \chi_{1} \chi_{2}\right)$ have

$$
\Re\left(F\left(\left(\beta_{1}+i \gamma_{1}+i \gamma_{2}-\rho\right) \mathcal{L}\right) \geq 0\right.
$$

Thus

$$
K\left(\beta_{1}+i \gamma_{1}+i \gamma_{2}, \chi_{1} \chi_{2}\right) \leq f(0)\left(\frac{1}{2} \phi\left(\chi_{1} \chi_{2}\right)+\varepsilon\right) \mathcal{L},
$$

by Lemma 5.2 , and similarly

$$
K\left(\beta_{1}+i \gamma_{1}-i \gamma_{2}, \chi_{1} \overline{\chi_{2}}\right) \leq f(0)\left(\frac{1}{2} \phi\left(\chi_{1} \overline{\chi_{2}}\right)+\varepsilon\right) \mathcal{L} .
$$

Here we need to observe that neither $\chi_{1} \chi_{2}$ nor $\chi_{1} \overline{\chi_{2}}$ is principal, since $\chi_{2}$ differs from $\chi_{1}$ and $\overline{\chi_{1}}$. By the same argument we have

$$
\Re\left(F\left(\left(\beta_{1}+i \gamma_{1}-\rho\right) \mathcal{L}\right)\right) \geq 0
$$


for all zeros in the disc

$$
\left|1+i \gamma_{1}-\rho\right| \leq \delta
$$

However $\rho_{1}$ falls in this set, so that

$$
\sum_{\left|1+i \gamma_{1}-\rho\right| \leq \delta} \Re\left(F\left(\left(\beta_{1}+i \gamma_{1}-\rho\right) \mathcal{L}\right)\right) \geq F(0),
$$

and hence

$$
K\left(\beta_{1}+i \gamma_{1}, \chi_{1}\right) \leq-\mathcal{L} F(0)+f(0)\left(\frac{1}{2} \phi\left(\chi_{1}\right)+\varepsilon\right) \mathcal{L}
$$

Similarly

$$
K\left(\beta_{1}+i \gamma_{2}, \chi_{2}\right) \leq-\mathcal{L} F\left(\lambda_{2}-\lambda_{1}\right)+f(0)\left(\frac{1}{2} \phi\left(\chi_{2}\right)+\varepsilon\right) \mathcal{L}
$$

Finally Lemma 5.3 yields

$$
K\left(\beta_{1}, \chi_{0}\right) \leq \mathcal{L} F\left(-\lambda_{1}\right)+f(0) \varepsilon \mathcal{L} .
$$

Gathering together the bounds $(6.4),(6.5),(6.6),(6.7),(6.8)$ and (6.9) we conclude that

$$
F\left(-\lambda_{1}\right)-F(0)-F\left(\lambda_{2}-\lambda_{1}\right)+f(0)(\psi+4 \varepsilon) \geq 0,
$$

with

$$
\psi=\frac{1}{2} \phi\left(\chi_{1}\right)+\frac{1}{2} \phi\left(\chi_{2}\right)+\frac{1}{4} \phi\left(\chi_{1} \chi_{2}\right)+\frac{1}{4} \phi\left(\chi_{1} \overline{\chi_{2}}\right) \leq \frac{1}{2} .
$$

We therefore have:

Lemma 6.2 Let $\lambda_{1}$ and $\lambda_{2}$ be defined as above, and let $f$ satisfy Conditions 1 and 2. Then

$$
F\left(-\lambda_{1}\right)-F(0)-F\left(\lambda_{2}-\lambda_{1}\right)+f(0)(\psi+\varepsilon) \geq 0
$$

for any $\varepsilon>0$, where $\psi$ is given by (6.10), providing that $q$ is sufficiently large.

We now consider a more complicated example. Suppose that $L\left(s, \chi_{1}\right)$ has a zero $\rho^{\prime} \neq \rho_{1}$ in the rectangle $R$, given by (6.1) (or a repeated zero $\rho^{\prime}=\rho_{1}$ ). In case $\chi_{1}$ is real and $\rho_{1}$ is complex we exclude also the value $\rho^{\prime}=\overline{\rho_{1}}$. Choose such a zero $\rho^{\prime}$ with $\Re\left(\rho^{\prime}\right)$ maximal, and put

$$
\rho^{\prime}=\beta^{\prime}+i \gamma^{\prime}, \beta^{\prime}=1-\mathcal{L}^{-1} \lambda^{\prime}, \lambda^{\prime}=\mathcal{L}^{-1} \mu^{\prime},
$$

in analogy to (6.2). We consider estimates for $\lambda^{\prime}$ in the case in which $\chi_{1}$ and $\rho_{1}$ are real. In view of the inequality

$$
\left\{1+\chi_{1}(n)\right\}\left\{1+\Re\left(\chi_{1}(n) n^{-i \gamma^{\prime}}\right)\right\} \geq 0
$$


we obtain

$$
K\left(\beta^{\prime}, \chi_{0}\right)+K\left(\beta^{\prime}, \chi_{1}\right)+K\left(\beta^{\prime}+i \gamma^{\prime}, \chi_{1}\right)+K\left(\beta^{\prime}+i \gamma^{\prime}, \chi_{0}\right) \geq 0 .
$$

To bound $K\left(\beta^{\prime}, \chi_{1}\right)$ using Lemma 5.2 , we must consider

$$
\sum_{|1-\rho| \leq \delta} \Re\left(F\left(\left(\beta^{\prime}-\rho\right) \mathcal{L}\right) .\right.
$$

By the choice of $\beta^{\prime}$, all terms $\rho \neq \rho_{1}$ produce non-negative contributions, so that they may be dropped. This yields

$$
K\left(\beta^{\prime}, \chi_{1}\right) \leq-\mathcal{L} F\left(\lambda_{1}-\lambda^{\prime}\right)+f(0)\left(\frac{1}{2} \phi\left(\chi_{1}\right)+\varepsilon\right) .
$$

In estimating $K\left(\beta^{\prime}+i \gamma^{\prime}, \chi_{1}\right)$ we examine

$$
\sum_{\left|1+i \gamma^{\prime}-\rho\right| \leq \delta} \Re\left(F\left(\left(\rho^{\prime}-\rho\right) \mathcal{L}\right) .\right.
$$

There will certainly be a term for $\rho=\rho^{\prime}$, whereas $\rho=\rho_{1}$ may or may not be present. However, if $\left|1+i \gamma^{\prime}-\rho_{1}\right|>\delta$, then

$$
F\left(\left(\rho^{\prime}-\rho_{1}\right) \mathcal{L}\right) \ll \mathcal{L}^{-1},
$$

by (5.3) and (5.4), suitably modified to allow $\Re(z) \leq 0$. Since each term in the sum is non-negative it follows that

$$
\sum_{\left|1+i \gamma^{\prime}-\rho\right| \leq \delta} \Re\left(F\left(\left(\rho^{\prime}-\rho\right) \mathcal{L}\right) \geq F(0)+\Re\left(F\left(\rho^{\prime}-\rho_{1}\right) \mathcal{L}\right)+O\left(\mathcal{L}^{-1}\right)\right.
$$

whether $\rho_{1}$ is present or not. We conclude that

$$
K\left(\beta^{\prime}+i \gamma^{\prime}, \chi_{1}\right) \leq-\mathcal{L} F(0)-\mathcal{L} \Re\left(F\left(\lambda_{1}-\lambda^{\prime}+i \mu^{\prime}\right)\right)+f(0)\left(\frac{1}{2} \phi\left(\chi_{1}\right)+2 \varepsilon\right),
$$

for large enough $q$. We also have

$$
K\left(\beta^{\prime}, \chi_{0}\right)=\mathcal{L} F\left(-\lambda^{\prime}\right)+o(1)
$$

and

$$
K\left(\beta^{\prime}+i \gamma^{\prime}, \chi_{0}\right)=\mathcal{L} \Re\left(F\left(-\lambda^{\prime}+i \mu^{\prime}\right)\right)+o(1),
$$

by Lemma 5.3. Combining the estimates $(6.11),(6.13),(6.15),(6.16)$ and $(6.17)$ yields

$$
\begin{aligned}
& F\left(-\lambda^{\prime}\right)-F\left(\lambda_{1}-\lambda^{\prime}\right)-F(0)+\Re\left\{F\left(-\lambda^{\prime}+i \mu^{\prime}\right)\right\} \\
& -\Re\left\{F\left(\lambda_{1}-\lambda^{\prime}+i \mu^{\prime}\right)\right\}+f(0)\left(\phi\left(\chi_{1}\right)+4 \varepsilon\right) \geq 0
\end{aligned}
$$


However

$$
\begin{aligned}
\Re\left\{F\left(-\lambda^{\prime}+i \mu^{\prime}\right)-F\left(\lambda_{1}-\lambda^{\prime}+i \mu^{\prime}\right)\right\} & =\int_{0}^{x_{0}} f(t) e^{\lambda^{\prime} t}\left(1-e^{-\lambda_{1} t}\right) \cos \mu^{\prime} t d t \\
& \leq \int_{0}^{x_{0}} f(t) e^{\lambda^{\prime} t}\left(1-e^{-\lambda_{1} t}\right) d t \\
& =F\left(-\lambda^{\prime}\right)-F\left(\lambda_{1}-\lambda^{\prime}\right) .
\end{aligned}
$$

We therefore have:

Lemma 6.3 Let $\chi_{1}$ and $\rho_{1}$ be real. Then

$$
2 F\left(-\lambda^{\prime}\right)-2 F\left(\lambda_{1}-\lambda^{\prime}\right)-F(0)+f(0)\left(\frac{1}{4}+\varepsilon\right) \geq 0
$$

for any $\varepsilon>0$, providing that $q$ is sufficiently large.

To get a more precise version of this result we may observe that (6.13) may be sharpened by including the contribution of the term $\rho=\rho^{\prime}$ to $(6.12)$, in the same way as we established (6.15). This yields

$$
K\left(\beta^{\prime}, \chi_{1}\right) \leq-\mathcal{L} F\left(\lambda_{1}-\lambda^{\prime}\right)-\mathcal{L} \Re\left(F\left(i \mu^{\prime}\right)\right)+f(0)\left(\frac{1}{2} \phi\left(\chi_{1}\right)+2 \varepsilon\right),
$$

so that (6.18) may be modified by including $-\mathcal{L} \Re\left(F\left(i \mu^{\prime}\right)\right)$ on the left. We therefore define

$$
F^{*}\left(\lambda_{1}, \lambda^{\prime}\right)=\max _{\mu^{\prime} \in \mathbb{R}} \Re\left\{F\left(-\lambda^{\prime}+i \mu^{\prime}\right)-F\left(\lambda_{1}-\lambda^{\prime}+i \mu^{\prime}\right)-F\left(i \mu^{\prime}\right)\right\}
$$

and conclude as follows.

Lemma 6.4 Let $\chi_{1}$ and $\rho_{1}$ be real.Then

$$
F\left(-\lambda^{\prime}\right)-F\left(\lambda_{1}-\lambda^{\prime}\right)-F(0)+F^{*}\left(\lambda_{1}, \lambda^{\prime}\right)+f(0)\left(\frac{1}{4}+\varepsilon\right) \geq 0
$$

for any $\varepsilon>0$, providing that $q$ is sufficiently large. 


\section{An Exercise in the Calculus of Variations}

The purpose of this section is to show how, for certain problems, one can choose the function $f$, used in the previous section, optimally. In Lemma 6.2, for example, one has

$$
F\left(-\lambda_{1}\right)-F\left(\lambda_{2}-\lambda_{1}\right)=\int_{0}^{\infty} \exp \left(\lambda_{1} t\right)\left\{1-\exp \left(-\lambda_{2} t\right)\right\} f(t) d t
$$

which is increasing with respect to $\lambda_{1}$. One then concludes, under the hypotheses of Lemma 6.2, that

$$
F\left(-\lambda_{2}\right)-2 F(0)+f(0)(\psi+\varepsilon) \geq 0 .
$$

We shall begin therefore by addresing the general problem of minimizing $F(-\lambda)$ for fixed values of $\lambda>0, F(0)(=A$, say) and $f(0)(=B$, say). We shall not pursue our argument with complete rigour, since it is merely our intention to motivate our choice of $f$, rather than to prove that it is indeed optimal.

We write $f(\lambda, A, B ; t)$ for the optimal function and $m(\lambda, A, B)$ for the corresponding minimum of $F(-\lambda)$. One readily sees that

$$
f(1, A, B ; \lambda t)=f\left(\lambda, \lambda^{-1} A, B ; t\right)
$$

and

$$
m(1, A, B)=\lambda m\left(\lambda, \lambda^{-1} A, B\right),
$$

so that it suffices to consider the case $\lambda=1$. In order to achieve Conditions 1 and 2 we shall take a continuous, non-negative, even function $g: \mathbb{R} \rightarrow \mathbb{R}$, supported in an interval $(-\gamma, \gamma)$, and we put $f=g * g$. We shall not impose any differentiability conditions on $g$, but it will turn out that our optimal function $f$ does indeed satisfy Condition 1 . As far as Condition 2 is concerned, $f$ will automatically be non-negative, since $g$ is. In order to show that $\Re(F) \geq 0$ for $\Re(z) \geq 0$ we may use Lemma 4.1 , taking $F_{1}=F$ and $F_{2}=0$. We know, from (5.3) and (5.4), that $F(z)$ tends to zero as $|z| \rightarrow \infty$ on $\mathcal{H}$. Moreover

$$
\Re(F(i y))=\int_{0}^{\infty} f(t) \cos (t y) d t=2\left(\int_{0}^{\infty} g(t) \cos (t y) d t\right)^{2} \geq 0
$$

for real $y$. Hence $\Re(F) \geq 0$ on $\mathcal{H}$ and Condition 2 holds.

In terms of $g$ we have

$$
\begin{aligned}
& A=F(0)=\frac{1}{2}\left(\int_{-\infty}^{\infty} g(t) d t\right)^{2} \\
& B=f(0)=\int_{-\infty}^{\infty} g(t)^{2} d t
\end{aligned}
$$


and

$$
F(-1)=\int_{0}^{\infty} e^{x} \int_{-\infty}^{\infty} g(t) g(x-t) d t d x .
$$

We now replace $g$ by $g+\varepsilon h$ for a small positive constant $\varepsilon$. We insist that $h$ is a continuous, even, real-valued function with compact support, and that $h$ is non-negative outside $(-\gamma, \gamma)$. Then $g+\varepsilon h$ satisfies the same conditions as $g$ (with a new value for $\gamma$ ), providing that $h / g$ is bounded below on $(-\gamma, \gamma)$, and that $\varepsilon$ is small enough. If we assume that

$$
\int_{-\infty}^{\infty} h(t) d t=0 \text { and } \int_{-\infty}^{\infty} h(t) g(t) d t=0
$$

then the value of $A$ is unaltered, while $B$ changes by $O\left(\varepsilon^{2}\right)$ only. However $F(-1)$ will be increased by

$$
\varepsilon \int_{-\infty}^{\infty} e^{|x|} \int_{-\infty}^{\infty} h(t) g(x-t) d t d x+O\left(\varepsilon^{2}\right) .
$$

Since $F(-1)$ is supposed to be minimal, we see that

$$
\int_{-\infty}^{\infty} h(t) g_{0}(t) d t \geq 0
$$

where

$$
g_{0}(t)=\int_{-\infty}^{\infty} e^{|x|} g(x-t) d x .
$$

Note that $g_{0}(t)$ is an even function of $t$. Since (7.5) must hold for all suitable $h$ satisfying (7.4) we conclude that

$$
g_{0}(t)=a+b g(t), \quad t \in(-\gamma, \gamma),
$$

and

$$
g_{0}(t) \geq a, \quad t \notin(-\gamma, \gamma),
$$

providing that $g$ is strictly positive on $(-\gamma, \gamma)$. We then have

$$
F(-1)=\frac{1}{2} \int_{-\infty}^{\infty} g(t) g_{0}(t) d t=a \sqrt{A / 2}+\frac{1}{2} b B .
$$

In view of (7.6) and (7.7) we have $g_{0}^{\prime \prime}=g_{0}+2 g$, and hence

$$
b g^{\prime \prime}(t)=(b+2) g(t)+a \quad(t \in(-\gamma, \gamma)) .
$$

Moreover $g$ is even, continuous, and vanishes at $\pm \gamma$. Thus, if $b=0$ then $g(t)=$ $-a / 2$, whence $g$ vanishes identically, and if $b=-2$, then

$$
g(t)=\frac{a}{4}\left(\gamma^{2}-t^{2}\right) \quad(-\gamma \leq t \leq \gamma) .
$$


For other values of $b$ we find

$$
g(t)=a b^{-1} \rho^{-2} \operatorname{sech}(\rho \gamma)\{\cosh \rho t-\cosh \rho \gamma\} \quad(-\gamma \leq t \leq \gamma),
$$

where

$$
\rho=\sqrt{(1+2 / b)}
$$

which may be real or pure imaginary. We feed this information back into (7.6) and obtain

$$
g_{0}(t)=a b^{-1} \rho^{-2} \operatorname{sech}(\rho \gamma)\left\{2 \cosh \rho \gamma+b \cosh \rho t+\rho e^{\gamma}\left(\frac{e^{-\rho \gamma}}{1-\rho}-\frac{e^{\rho \gamma}}{1+\rho}\right) \cosh t\right\}
$$

for $t \in(-\gamma, \gamma)$ and $b \neq 0,-2$. If we compare this with (7.7) we deduce that

$$
\rho e^{\gamma}\left(\frac{e^{-\rho \gamma}}{1-\rho}-\frac{e^{\rho \gamma}}{1+\rho}\right)=0
$$

and hence that

$$
\tanh \rho \gamma=\rho .
$$

Similarly, when $b=-2$ one finds that $\gamma=1$. Since

$$
\sinh \rho \gamma=\frac{\rho}{\sqrt{1-\rho^{2}}}, \quad \cosh \rho \gamma=\frac{1}{\sqrt{1-\rho^{2}}}
$$

we may now calculate, using (7.2),(7.3),(7.9) and (7.11) that

$$
\begin{aligned}
F(0) & =2\left(\frac{a}{b+2}\right)^{2}(1-\gamma)^{2}, \\
f(0) & =\left(\frac{a}{b+2}\right)^{2}\left\{3 \gamma-3-\rho^{2} \gamma\right\},
\end{aligned}
$$

and

$$
F(-1)=\left(\frac{a}{b+2}\right)^{2}\left(1-\rho^{2}\right)^{-1}\left\{3-2 \rho^{2}+3 \gamma \rho^{2}-3 \gamma\right\},
$$

for $b \neq 0,-2$; and similarly

$$
F(0)=\frac{a^{2}}{18}, f(0)=\frac{a^{2}}{15}, F(-1)=\frac{a^{2}}{10}
$$

for $b=-2$.

We now consider the conditions (7.12) and (7.13). If $b<-2$ then $0<1+2 / b<1$, so that $\rho$ is real and $0<\rho<1$. The equation (7.13) therefore has a unique solution for $\gamma$. If $-2<b<0$ then $1+2 / b<0$, so that $\rho$ is pure imaginary. Putting $\rho=i \zeta$ we have $\tan \zeta \gamma=\zeta$, by (7.13), and since

$$
g(t)=a b^{-1} \rho^{-2} \sec (\zeta \gamma)\{\cos \zeta t-\cos \zeta \gamma\} \quad(-\gamma \leq t \leq \gamma)
$$


has to be non-negative, we conclude that $0<\zeta \gamma<\pi$. Then $\tan \zeta \gamma=\zeta$ must be positive, so that $0<\zeta \gamma<\pi / 2$. Finally, if $b>0$, then $\rho$ would be real and greater than 1 , whence (7.13) is insoluble.

We now go back to (7.11),(7.14),(7.15) and (7.16) and substitute $t \lambda$ for $t, i \zeta / \lambda$ for $\rho$ and $\gamma \lambda$ for $\gamma$. After removing a constant of proportionality we obtain the following.

Lemma 7.1 Let $0<\theta<\pi / 2$, and let $\lambda>0$. Put $\zeta=\lambda \tan \theta, \gamma=\theta / \zeta$ and

$$
g(t)=\lambda\left(1+\tan ^{2} \theta\right)(\cos \zeta t-\cos \theta) \quad(-\gamma \leq t \leq \gamma),
$$

and $g(t)=0$ for $|t| \geq \gamma$. Then

$$
\begin{array}{r}
f(x)=\lambda\left(1+\tan ^{2} \theta\right)\left[\lambda\left(1+\tan ^{2} \theta\right)\left(\gamma-\frac{1}{2} x\right) \cos \zeta x+\lambda(2 \gamma-x)\right. \\
\left.+\frac{\sin (2 \theta-\zeta x)}{\sin 2 \theta}-2\left(1+\frac{\sin (\theta-\zeta x)}{\sin \theta}\right)\right]
\end{array}
$$

if $0 \leq x \leq 2 \gamma$, and $f(x)=0$ for $x \geq 2 \gamma$. Moreover

$$
\begin{aligned}
f(0) & =\lambda\left(1+\tan ^{2} \theta\right)[\theta \tan \theta+3 \theta \cot \theta-3] \\
F(0) & =2\left(1+\tan ^{2} \theta\right)[1-\theta \cot \theta]^{2}
\end{aligned}
$$

and

$$
F(-\lambda)=2 \tan ^{2} \theta+3-3 \theta \tan \theta-3 \theta \cot \theta .
$$

Lemma 7.2 Put $\gamma=\lambda^{-1}$ and

$$
g(t)=\lambda\left(1-\lambda^{2} t^{2}\right) \quad(-\gamma \leq t \leq \gamma)
$$

and $g(t)=0$ for $|t| \geq \gamma$. Then

$$
f(x)=\frac{\lambda}{30}(2-\lambda x)^{3}\left[4+6 \lambda x+\lambda^{2} x^{2}\right] \quad(0 \leq x \leq 2 \gamma)
$$

and $f(x)=0$ for $x \geq 2 \gamma$. Moreover $f(0)=16 \lambda / 15, F(0)=8 / 9$ and $F(-\lambda)=$ $8 / 5$.

Lemma 7.3 Let $\theta>0$, and take $\lambda>0$. Put $\rho=\lambda \tanh \theta, \gamma=\theta / \rho$, and

$$
g(t)=\lambda\left(1-\tanh ^{2} \theta\right)(\cosh \theta-\cosh \rho t) \quad(-\gamma \leq t \leq \gamma),
$$

and $g(t)=0$ for $|t| \geq \gamma$. Then

$$
\begin{array}{r}
f(x)=\lambda\left(1-\tanh ^{2} \theta\right)\left[\lambda\left(1-\tanh ^{2} \theta\right)\left(\gamma-\frac{1}{2} x\right) \cosh \rho x+\lambda(2 \gamma-x)\right. \\
\left.+\frac{\sinh (2 \theta-\rho x)}{\sinh 2 \theta}-2\left(1+\frac{\sinh (\theta-\rho x)}{\sinh \theta}\right)\right]
\end{array}
$$


if $0 \leq x \leq 2 \gamma$, and $f(x)=0$ for $x \geq 2 \gamma$. Moreover

$$
\begin{aligned}
& f(0)=\lambda\left(1-\tanh ^{2} \theta\right)[3 \theta \operatorname{coth} \theta-\theta \tanh \theta-3] \\
& F(0)=2\left(1-\tanh ^{2} \theta\right)[1-\theta \operatorname{coth} \theta]^{2}
\end{aligned}
$$

and

$$
F(-\lambda)=3-2 \tanh ^{2} \theta+3 \theta \tanh \theta-3 \theta \operatorname{coth} \theta .
$$

Lemma 7.2 can be viewed as the limiting case $\theta \rightarrow 0$ of Lemmas 7.1 and 7.3 . Of course we expect that the functions $f$ are extremal in an appropriate sense, but we do not claim this formally.

We may consider the problem of maximizing $F(\lambda)$ similarly. The argument is closely analogous, and leads to:-

Lemma 7.4 Let $\pi / 2<\theta<\pi$ and take $\lambda>0$. Put $\zeta=-\lambda \tan \theta, \gamma=\theta / \zeta$ and

$$
g(t)=\lambda\left(1+\tan ^{2} \theta\right)(\cos \zeta t-\cos \theta) \quad(-\gamma \leq t \leq \gamma)
$$

and $g(t)=0$ for $|t| \geq \gamma$. Then

$$
\begin{array}{r}
f(x)=\lambda\left(1+\tan ^{2} \theta\right)\left[\lambda\left(1+\tan ^{2} \theta\right)\left(\gamma-\frac{1}{2} x\right) \cos \zeta x+\lambda(2 \gamma-x)\right. \\
\left.-\frac{\sin (2 \theta-\zeta x)}{\sin 2 \theta}+2\left(1+\frac{\sin (\theta-\zeta x)}{\sin \theta}\right)\right]
\end{array}
$$

if $0 \leq x \leq 2 \gamma$, and $f(x)=0$ for $x \geq 2 \gamma$. Moreover

$$
\begin{aligned}
& f(0)=\lambda\left(1+\tan ^{2} \theta\right)[3-\theta \tan \theta-3 \theta \cot \theta] \\
& F(0)=2\left(1+\tan ^{2} \theta\right)[1-\theta \cot \theta]^{2}
\end{aligned}
$$

and

$$
F(\lambda)=2 \tan ^{2} \theta+3-3 \theta \tan \theta-3 \theta \cot \theta .
$$

It should be noted that $f(x)$ does indeed satisfy Condition 1 in every case.

In view of (7.1) we now examine the problem of minimizing

$$
\{F(-\lambda)-k F(0)\} / f(0)
$$

where $k$ is a positive constant. If we substitute from Lemma 7.1, for example we can check that the derivative with respect to $\theta$ vanishes when

$$
\sin ^{2} \theta=k(1-\theta \cot \theta) \text {. }
$$

This requires $0<k<3$, and gives

$$
F(-\lambda)-k F(0)=-\lambda^{-1} f(0) \cos ^{2} \theta .
$$

Here $\theta \rightarrow 0$ as $k \rightarrow 3$, giving the case considered in Lemma 7.2. For Lemma 7.3 a similar argument applies. This leads to:- 
Lemma 7.5 If $0<k<3$ and $\sin ^{2} \theta=k(1-\theta \cot \theta)$ with $0<\theta<\pi / 2$, then the function of Lemma 7.1 has

$$
F(-\lambda)-k F(0)=-\lambda^{-1} f(0) \cos ^{2} \theta .
$$

Similarly, for $k>3$ and $\sinh ^{2} \theta=k(\theta \operatorname{coth} \theta-1)$, the function of Lemma 7.3 has

$$
F(-\lambda)-k F(0)=-\lambda^{-1} f(0) \cosh ^{2} \theta .
$$

Finally, when $k=3$, the function of Lemma 7.2 has

$$
F(-\lambda)-k F(0)=-\lambda^{-1} f(0) .
$$




\section{The Deuring-Heilbronn Phenomenon}

We now use the work of the preceding sections to give lower bounds for $\lambda^{\prime}$ and $\lambda_{2}$ in terms of $\lambda_{1}$, under the assumption that $\chi_{1}$ and $\rho_{1}$ are real. We shall use three alternative methods, depending on the size of $\lambda_{1}$.

Firstly we investigate the case in which $\lambda_{1}$ is very small. Here we apply Lemma 6.3, together with the observation that

$$
\begin{aligned}
F\left(-\lambda^{\prime}\right)-F\left(\lambda_{1}-\lambda^{\prime}\right) & =\int_{0}^{\infty} f(t) e^{\lambda^{\prime} t}\left(1-e^{-\lambda_{1} t}\right) d t \\
& \leq \lambda_{1} \int_{0}^{\infty} t f(t) e^{\lambda^{\prime} t} d t .
\end{aligned}
$$

We content ourselves with the simplest choice of $f(t)$, namely

$$
f(t)=\left\{\begin{array}{cc}
x_{0}-t, & 0 \leq t \leq x_{0}, \\
0, & t \geq 0,
\end{array}\right.
$$

for which Conditions 1 and 2 are easily verified. Then

$$
\begin{aligned}
\int_{0}^{x_{0}} t\left(x_{0}-t\right) e^{\lambda^{\prime} t} d t & =\lambda^{\prime-3}\left(x_{0} \lambda^{\prime} e^{x_{0} \lambda^{\prime}}-2 e^{x_{0} \lambda^{\prime}}+x_{0} \lambda^{\prime}+2\right) \\
& \leq \lambda^{\prime-3}\left(x_{0} \lambda^{\prime} e^{x_{0} \lambda^{\prime}}\right)
\end{aligned}
$$

so that

$$
2 F\left(-\lambda^{\prime}\right)-2 F\left(\lambda_{1}-\lambda^{\prime}\right) \leq 2 x_{0} \lambda_{1} \lambda^{\prime-2} e^{x_{0} \lambda^{\prime}}
$$

Moreover

$$
F(0)=\int_{0}^{x_{0}}\left(x_{0}-t\right) d t=\frac{1}{2} x_{0}^{2},
$$

and $f(0)=x_{0}$. Lemma 6.3 then yields

$$
2 x_{0} \lambda_{1} \lambda^{\prime-2} e^{x_{0} \lambda^{\prime}}-\frac{1}{2} x_{0}^{2}+x_{0}\left(\frac{1}{4}+\varepsilon\right) \geq 0 .
$$

We choose $x_{0}=\frac{1}{2}+\lambda^{\prime-1}+2 \varepsilon$, so that the dependence on $f$ implied by the condition " $q$ sufficiently large" will be uniform for $\lambda^{\prime} \geq 1$, say. Now (8.1) leads to

$$
\lambda_{1} \geq \frac{\lambda^{\prime}}{4 e} \exp \left(-\left(\frac{1}{2}+2 \varepsilon\right) \lambda^{\prime}\right) \geq \exp \left(-\left(\frac{1}{2}+2 \varepsilon\right) \lambda^{\prime}\right)
$$

for $\lambda^{\prime} \geq 4 e$ and $q \geq q(\varepsilon)$. We therefore conclude:-

Lemma 8.1 Under the conditions of Lemma 6.3, either $\lambda^{\prime}<4 e$ or, for any $\varepsilon>0$, we have

$$
\lambda^{\prime} \geq(2-\varepsilon) \log \left(\lambda_{1}^{-1}\right)
$$

for $q \geq q(\varepsilon)$. 
We turn now to the middle range for $\lambda_{1}$. Here we again apply Lemma 6.3, but this time we use the function of Lemmas 7.1 and 7.5 corresponding to $k=\frac{3}{2}$. Numerical experiment suggests that this function, which is, of course, optimal when $\lambda_{1}=\lambda^{\prime}$, is a good choice throughout the relevant range for $\lambda_{1}$. In order to specify $f$ we must also select $\lambda$, and we make a variety of choices, depending on the size of $\lambda_{1}$. If $\lambda_{1}$ lies in an interval $0 \leq \lambda_{1} \leq b$, and $\lambda=\lambda(b)$ is specified, then the function

$$
F\left(-\lambda^{\prime}\right)-F\left(\lambda_{1}-\lambda^{\prime}\right)=\int_{0}^{\infty} f(t) e^{\lambda^{\prime} t}\left(1-e^{-\lambda_{1} t}\right) d t
$$

is increasing with respect to both $\lambda_{1}$ and $\lambda^{\prime}$. If we choose $\lambda_{b}^{\prime}$ to give

$$
2 F\left(-\lambda_{b}^{\prime}\right)-2 F\left(b-\lambda_{b}^{\prime}\right)-F(0)+\frac{1}{4} f(0)=0
$$

it then follows that $\lambda^{\prime} \geq \lambda_{b}^{\prime}-\varepsilon$ for $q \geq q(\varepsilon, b)$, whenever $0 \leq \lambda_{1} \leq b$. Table 2 gives values for $b$ (as " $\lambda_{1}$ "), for $\lambda(b)$ (as " $\lambda$ ") and calculated values a little below $\lambda_{b}^{\prime}\left(\right.$ as $" \lambda^{\prime \prime}$ "), together with $2 \log (1 / b)$ (as " $2 \log 1 / \lambda_{1}$ "). Similarly for Table 3 . In particular one sees that $\lambda^{\prime} \geq 4 e$ for $\lambda_{1} \leq 0.006$, and that (8.2) holds for $0.006 \leq \lambda_{1} \leq 0.2$. Moreover the weaker bound

$$
\lambda^{\prime} \geq \frac{3}{2} \log \lambda_{1}^{-1}
$$

holds for $0.2 \leq \lambda_{1} \leq 0.4$.

Lemma 6.3 is inefficient for larger values of $\lambda_{1}$, and instead we use our third method. However we must first dispose of the case in which $\mu^{\prime}=0$. Here Lemma 6.4 reduces to

$$
2\left[F\left(-\lambda^{\prime}\right)-F\left(\lambda_{1}-\lambda^{\prime}\right)-F(0)\right]+f(0)\left(\frac{1}{4}+\varepsilon\right) \geq 0,
$$

whence

$$
F\left(-\lambda^{\prime}\right)-2 F(0)+f(0)\left(\frac{1}{8}+\varepsilon\right) \geq 0 .
$$

We apply this using the function from Lemmas 7.1 and 7.5 corresponding to $k=2$. Thus

$$
\lambda^{\prime-1} \cos ^{2} \theta \leq \frac{1}{8}+\varepsilon \text {. }
$$

For $k=2$ we have $\theta=0.9873 \ldots$, whence $\lambda^{\prime} \geq 2.427 \ldots$

Lemma 8.2 Let $\chi_{1}, \rho_{1}$ and $\rho^{\prime}$ be real. Then $\lambda^{\prime} \geq 2.427 \ldots$

When $\mu^{\prime} \neq 0$ we use the inequality

$$
\begin{aligned}
0 \leq & \chi_{0}(n)\left(1+\chi_{1}(n)\right)\left(K+\Re\left\{\chi_{1}(n) n^{-i \gamma^{\prime}}\right\}\right)^{2} \\
= & \left(K^{2}+\frac{1}{2}\right)\left\{\chi_{0}(n)+\chi_{1}(n)\right\}+2 K\left\{\Re\left(\chi_{0}(n) n^{-i \gamma^{\prime}}\right)+\Re\left(\chi_{1}(n) n^{-i \gamma^{\prime}}\right)\right\} \\
& +\frac{1}{2}\left\{\Re\left(\chi_{0}(n) n^{-2 i \gamma^{\prime}}\right)+\Re\left(\chi_{1}(n) n^{-2 i \gamma^{\prime}}\right)\right\},
\end{aligned}
$$




\begin{tabular}{ccrl}
$\lambda_{1}$ & \multicolumn{1}{c}{$\lambda$} & \multicolumn{1}{c}{$\lambda^{\prime}$} & \multicolumn{1}{c}{$2 \log 1 / \lambda_{1}$} \\
\hline 0.006 & 1.10 & 11.34 & 10.23 \\
0.008 & 1.09 & 10.71 & 9.65 \\
0.010 & 1.08 & 10.21 & 9.21 \\
0.015 & 1.07 & 9.31 & 8.39 \\
0.020 & 1.06 & 8.66 & 7.82 \\
0.025 & 1.05 & 8.15 & 7.37 \\
0.030 & 1.04 & 7.73 & 7.01 \\
0.035 & 1.03 & 7.37 & 6.70 \\
0.040 & 1.025 & 7.06 & 6.43 \\
0.045 & 1.015 & 6.79 & 6.20 \\
0.05 & 1.010 & 6.54 & 5.99 \\
0.06 & 1.000 & 6.11 & 5.62 \\
0.07 & 0.895 & 5.75 & 5.31 \\
0.08 & 0.975 & 5.43 & 5.05 \\
0.09 & 0.970 & 5.16 & 4.81 \\
0.10 & 0.965 & 4.96 & 4.60 \\
0.11 & 0.960 & 4.74 & 4.41 \\
0.12 & 0.952 & 4.53 & 4.24 \\
0.13 & 0.945 & 4.35 & 4.08 \\
0.14 & 0.939 & 4.18 & 3.93 \\
0.15 & 0.932 & 4.02 & 3.79 \\
0.16 & 0.952 & 3.87 & 3.66 \\
0.17 & 0.919 & 3.73 & 3.54 \\
0.18 & 0.912 & 3.59 & 3.42 \\
0.19 & 0.905 & 3.47 & 3.32 \\
0.20 & 0.899 & 3.35 & 3.21
\end{tabular}

Table 2: $\lambda^{\prime}$ for real $\chi_{1}$ and $\rho_{1}$

\begin{tabular}{cccl}
$\lambda_{1}$ & $\lambda$ & $\lambda^{\prime}$ & $\frac{3}{2} \log 1 / \lambda_{1}$ \\
\hline 0.20 & 0.899 & 3.35 & 2.41 \\
0.25 & 0.873 & 2.84 & 2.77 \\
0.30 & 0.847 & 2.43 & 1.80 \\
0.35 & 0.824 & 2.09 & 1.57 \\
0.40 & 0.807 & 1.80 & 1.37
\end{tabular}

Table 3: $\lambda^{\prime}$ for real $\chi_{1}$ and $\rho_{1}$ 
together with the ideas of $\S 4$. Thus if

$$
p(X)=\sum a_{k} X^{k}
$$

is one of the polynomials (4.12), we consider

$$
\mathcal{L}^{-1} \sum_{k} a_{k} \frac{\left(a+\lambda_{1}\right)^{k}}{(k-1) !} \sum_{n=1}^{\infty} \Lambda(n) \Re\left(\frac{\chi(n)}{n^{s}}\right)\left(\mathcal{L}^{-1} \log n\right)^{k-1}=\Sigma(s, \chi),
$$

say. Then

$$
\begin{aligned}
0 \leq\left(K^{2}+\frac{1}{2}\right)\left\{\Sigma\left(\sigma, \chi_{0}\right)+\right. & \left.\Sigma\left(\sigma, \chi_{1}\right)\right\}+2 K\left\{\Sigma\left(\sigma+i \gamma^{\prime}, \chi_{0}\right)+\Sigma\left(\sigma+i \gamma^{\prime}, \chi_{1}\right)\right\} \\
+ & \frac{1}{2}\left\{\Sigma\left(\sigma+2 i \gamma^{\prime}, \chi_{0}\right)+\Sigma\left(\sigma+2 i \gamma^{\prime}, \chi_{1}\right)\right\} .
\end{aligned}
$$

However, taking $s=\sigma+i t, \sigma=1+\mathcal{L}^{-1} a$, we have

$$
\Sigma(s, \chi)=\Re\left\{\sum_{k} a_{k} \frac{(-1)^{k}}{(k-1) !}\left(\frac{a+\lambda_{1}}{\mathcal{L}}\right)^{k} \frac{d^{k-1}}{d s^{k-1}}\left(\frac{L^{\prime}}{L}(s, \chi)\right)\right\} .
$$

For $\chi=\chi_{0}$ this is merely

$$
p\left(\frac{a+\lambda_{1}}{(s-1) \mathcal{L}}\right)+o(1) .
$$

When $\chi \neq \chi_{0}$ and $k \geq 2$ we have

$$
\begin{aligned}
\frac{(-1)^{k}}{(k-1) !} \mathcal{L}^{-k} \frac{d^{k-1}}{d s^{k-1}}\left(\frac{L^{\prime}}{L}(s, \chi)\right) & =-\mathcal{L}^{-k} \sum_{\rho}\left(\frac{1}{s-\rho}\right)^{k}+o(1) \\
& =-\mathcal{L}^{-k} \sum_{|1+i t-\rho| \leq \delta}\left(\frac{1}{s-\rho}\right)^{k}+o(1)
\end{aligned}
$$

for any fixed $\delta>0$, since

$$
\sum_{|1+i t-\rho|>\delta}|s-\rho|^{-k} \ll \sum_{|1+i t-\rho|>\delta}|s-\rho|^{-2} \ll \sum_{\rho} \frac{1}{1+|s-\rho|^{2}} \ll \mathcal{L} .
$$

Finally, for $\chi \neq \chi_{0}, k=1$, we may use Lemma 3.1. We conclude that

$$
\Sigma(s, \chi) \leq-\sum_{|1+i t-\rho| \leq \delta} \Re\left(p\left(\frac{a+\lambda_{1}}{(s-\rho) \mathcal{L}}\right)\right)+a_{1}\left(a+\lambda_{1}\right)\left(\frac{\phi}{2}+\varepsilon\right)
$$

for $\chi \neq \chi_{0}$ and sufficiently large $q$. We now observe that

$$
\Re\left(p\left(\frac{a+\lambda_{1}}{(s-\rho) \mathcal{L}}\right)\right) \geq 0
$$


for all relevant zeros $\rho$, by virtue of (4.11). Moreover, if $|1+i t-\rho| \geq \delta$, then

$$
\Re\left(p\left(\frac{a+\lambda_{1}}{(s-\rho) \mathcal{L}}\right)\right)=O\left(\mathcal{L}^{-1}\right)
$$

Hence

$$
\Sigma\left(\sigma, \chi_{1}\right) \leq-\Re\left(p\left(\frac{a+\lambda_{1}}{\left(\sigma-\rho_{1}\right) \mathcal{L}}\right)\right)-\Re\left(p\left(\frac{a+\lambda_{1}}{\left(\sigma-\rho^{\prime}\right) \mathcal{L}}\right)\right)+a_{1}\left(a+\lambda_{1}\right)\left(\frac{\phi}{2}+2 \varepsilon\right),
$$

whether $\left|1-\rho^{\prime}\right| \leq \delta$ or not. By this argument we find that

$$
\begin{gathered}
\Sigma\left(\sigma, \chi_{1}\right) \leq-p(1)-\Re\left(p\left(\frac{a+\lambda_{1}}{a+\lambda^{\prime}+i \mu^{\prime}}\right)\right)+a_{1}\left(a+\lambda_{1}\right)\left(\frac{\phi}{2}+2 \varepsilon\right), \\
\Sigma\left(\sigma+i \gamma^{\prime}, \chi_{1}\right) \leq-p\left(\frac{a+\lambda_{1}}{a+\lambda^{\prime}}\right)-\Re\left(p\left(\frac{a+\lambda_{1}}{a+\lambda_{1}+i \mu^{\prime}}\right)\right)-\Re\left(p\left(\frac{a+\lambda_{1}}{a+\lambda^{\prime}+2 i \mu^{\prime}}\right)\right) \\
+a_{1}\left(a+\lambda_{1}\right)\left(\frac{\phi}{2}+2 \varepsilon\right),
\end{gathered}
$$

if $\mu^{\prime} \neq 0$, and

$$
\begin{aligned}
\Sigma\left(\sigma+2 i \gamma^{\prime}, \chi_{1}\right) \leq-\Re\left(p\left(\frac{a+\lambda_{1}}{a+\lambda^{\prime}+i \mu^{\prime}}\right)\right)- & \Re\left(p\left(\frac{a+\lambda_{1}}{a+\lambda_{1}+2 i \mu^{\prime}}\right)\right) \\
& +a_{1}\left(a+\lambda_{1}\right)\left(\frac{\phi}{2}+2 \varepsilon\right) .
\end{aligned}
$$

The inequality (8.5) now yields

$$
\begin{array}{r}
0 \leq\left(K^{2}+\frac{1}{2}\right)\left(p\left(\frac{a+\lambda_{1}}{a}\right)-\right. \\
p(1))-2 K p\left(\frac{a+\lambda_{1}}{a+\lambda^{\prime}}\right)-A-B \\
+a_{1}\left(a+\lambda_{1}\right)(K+1)^{2}\left(\frac{\phi}{2}+3 \varepsilon\right),
\end{array}
$$

where

$$
\begin{aligned}
A & =\Re\left\{\left(K^{2}+1\right) p\left(\frac{a+\lambda_{1}}{a+\lambda^{\prime}+i \mu^{\prime}}\right)+2 K p\left(\frac{a+\lambda_{1}}{a+\lambda_{1}+i \mu^{\prime}}\right)-2 K p\left(\frac{a+\lambda_{1}}{a+i \mu^{\prime}}\right)\right\} \\
& \geq 2 K \Re\left\{p\left(\frac{a+\lambda_{1}}{a+\lambda^{\prime}+i \mu^{\prime}}\right)+p\left(\frac{a+\lambda_{1}}{a+\lambda_{1}+i \mu^{\prime}}\right)-p\left(\frac{a+\lambda_{1}}{a+i \mu^{\prime}}\right)\right\}
\end{aligned}
$$

and

$$
\begin{aligned}
B & =\Re\left\{2 K p\left(\frac{a+\lambda_{1}}{a+\lambda^{\prime}+2 i \mu^{\prime}}\right)+\frac{1}{2} p\left(\frac{a+\lambda_{1}}{a+\lambda_{1}+2 i \mu^{\prime}}\right)-\frac{1}{2} p\left(\frac{a+\lambda_{1}}{a+2 i \mu^{\prime}}\right)\right\} \\
& \geq \frac{1}{2} \Re\left\{p\left(\frac{a+\lambda_{1}}{a+\lambda^{\prime}+2 i \mu^{\prime}}\right)+p\left(\frac{a+\lambda_{1}}{a+\lambda_{1}+2 i \mu^{\prime}}\right)-p\left(\frac{a+\lambda_{1}}{a+2 i \mu^{\prime}}\right)\right\}
\end{aligned}
$$


providing that $K \geq \frac{1}{4}$. In order to check whether or not $A, B \geq 0$ we shall focus on the third of the polynomials (4.12), $P_{3}(X)$, say. This turns out to give the best estimates. We have

$$
\begin{aligned}
& \Re\left(P_{3}\left(\frac{\alpha}{\beta+i t}\right)\right)= \\
& \quad \frac{8}{3}\left(\frac{\alpha \beta}{\beta^{2}+t^{2}}\right)^{3}+\frac{\alpha\left\{\beta^{3}(\beta+2 \alpha)+\beta(\beta+\alpha) t^{2}+t^{4}\right\}}{\left(\beta^{2}+t^{2}\right)^{3}}(\beta-\alpha),
\end{aligned}
$$

whence

$$
\Re\left(P_{3}\left(\frac{\alpha}{\beta+i t}\right)\right) \quad\left\{\begin{array}{l}
\geq \\
\leq
\end{array}\right\} \frac{8}{3}\left(\frac{\alpha \beta}{\beta^{2}+t^{2}}\right)^{3} \quad\left\{\begin{array}{l}
\beta \geq \alpha, \\
\beta \leq \alpha,
\end{array}\right.
$$

so that

$$
\begin{aligned}
& \Re\left(P_{3}\left(\frac{a+\lambda_{1}}{a+\lambda^{\prime}+i \mu^{\prime}}\right)\right) \geq \frac{8}{3}\left(a+\lambda_{1}\right)^{3}\left(\frac{a+\lambda^{\prime}}{\left(a+\lambda^{\prime}\right)^{2}+\mu^{\prime 2}}\right)^{3}, \\
& \Re\left(P_{3}\left(\frac{a+\lambda_{1}}{a+\lambda_{1}+i \mu^{\prime}}\right)\right)=\frac{8}{3}\left(a+\lambda_{1}\right)^{3}\left(\frac{a+\lambda_{1}}{\left(a+\lambda_{1}\right)^{2}+\mu^{\prime 2}}\right)^{3}
\end{aligned}
$$

and

$$
\Re\left(P_{3}\left(\frac{a+\lambda_{1}}{a+i \mu^{\prime}}\right)\right) \leq \frac{8}{3}\left(a+\lambda_{1}\right)^{3}\left(\frac{a}{a^{2}+\mu^{\prime 2}}\right)^{3} .
$$

It follows that

$$
A \geq 2 K \frac{8}{3}\left(a+\lambda_{1}\right)^{3}\left(\frac{1}{a^{2}+\mu^{\prime 2}}\right)^{3} A_{1}
$$

with

$$
A_{1}=\left(a+\lambda^{\prime}\right)^{3}\left(\frac{a^{2}+\mu^{\prime 2}}{\left(a+\lambda^{\prime}\right)^{2}+\mu^{\prime 2}}\right)^{3}+\left(a+\lambda_{1}\right)^{3}\left(\frac{a^{2}+\mu^{\prime 2}}{\left(a+\lambda_{1}\right)^{2}+\mu^{\prime 2}}\right)^{3}-a^{3} .
$$

Here $A_{1}$ is increasing with respect to $\mu^{\prime} \geq 0$, so that

$$
\begin{aligned}
A_{1} & \geq\left(a+\lambda^{\prime}\right)^{3}\left(\frac{a^{2}}{\left(a+\lambda^{\prime}\right)^{2}}\right)^{3}+\left(a+\lambda_{1}\right)^{3}\left(\frac{a^{2}}{\left(a+\lambda_{1}\right)^{2}}\right)^{3}-a^{3} \\
& =a^{6}\left\{\left(a+\lambda^{\prime}\right)^{-3}+\left(a+\lambda_{1}\right)^{-3}-a^{-3}\right\} .
\end{aligned}
$$

Thus $A \geq 0$ providing that

$$
\left(a+\lambda^{\prime}\right)^{-3}+\left(a+\lambda_{1}\right)^{-3} \geq a^{-3} .
$$

Similarly we find that $B \geq 0$ providing that (8.6) holds. We may now conclude as follows. 
Lemma 8.3 Let $\chi_{1}$ and $\rho_{1}$ be real. Then

$$
\begin{array}{r}
\left(K^{2}+\frac{1}{2}\right)\left(P_{3}\left(\frac{a+\lambda_{1}}{a}\right)-P_{3}(1)\right)-2 K P_{3}\left(\frac{a+\lambda_{1}}{a+\lambda^{\prime}}\right)+(K+1)^{2}\left(a+\lambda_{1}\right)\left(\frac{1}{8}+\varepsilon\right) \\
\geq 0
\end{array}
$$

for any constants $a>0, K \geq \frac{1}{4}$ for which (8.6) holds, providing that $\rho_{1}$ is complex and $q \geq q(a, K, \varepsilon)$.

The left hand side of (8.7) is clearly increasing with $\lambda^{\prime}$. The derivative with respect to $\lambda_{1}$ is

$$
\begin{aligned}
& \frac{K^{2}+\frac{1}{2}}{a} P_{3}^{\prime}\left(\frac{a+\lambda_{1}}{a}\right)-\frac{2 K}{a+\lambda^{\prime}} P_{3}^{\prime}\left(\frac{a+\lambda_{1}}{a+\lambda^{\prime}}\right)+(K+1)^{2}\left(\frac{1}{8}+\varepsilon\right) \\
& \geq \frac{K^{2}+\frac{1}{2}}{a}\left\{1+2\left(\frac{a+\lambda_{1}}{a}\right)+2\left(\frac{a+\lambda_{1}}{a}\right)^{2}\right\}-\frac{2 K}{a+\lambda_{1}} P_{3}^{\prime}(1)+\frac{(K+1)^{2}}{8} .
\end{aligned}
$$

Hence the left hand side of (8.7) increases with $\lambda_{1}$ if

$$
\frac{K^{2}+\frac{1}{2}}{a}\left\{1+2\left(\frac{a+\lambda_{1}}{a}\right)+2\left(\frac{a+\lambda_{1}}{a}\right)^{2}\right\}+\frac{(K+1)^{2}}{8} \geq \frac{10 K}{a+\lambda_{1}} .
$$

Thus if we consider an interval $A \leq \lambda_{1} \leq B$, and choose $a, K$ optimally at $\lambda_{1}=$ $B$, the resulting value for $\lambda^{\prime}$ will be valid throughout $[A, B]$, providing that (8.8) holds at $\lambda_{1}=A$. Similarly, to check (8.6) over $[A, B]$ we use $\lambda_{1}=B$ together with the value of $\lambda^{\prime}$ corresponding to $\lambda_{1}=A$. A table of values $\lambda_{1}, \lambda^{\prime}, a, K$ obtained in this way is given as Table 4 . Thus the entry $0.7,1.724,4.5,0.85$ indicates that $\lambda^{\prime} \geq 1.724$ whenever $\lambda_{1} \leq 0.7$. One sees that Lemma 8.3 is sharper than Lemma 8.1 for values of $\lambda_{1}$ from around 0.35 onwards, and that Lemma 8.3 is effective up to $\lambda_{1}=1.294$, so that $\lambda^{\prime} \geq 1.294$ in all cases. Moreover we can check that (8.3) holds for $\lambda_{1} \geq 0.4$, since $\lambda^{\prime} \geq \lambda_{1}$ by definition. We summarize these conclusions as follows.

Lemma 8.4 Let $\chi_{1}$ and $\rho_{1}$ be real, and let $\varepsilon>0$. Then

$$
\lambda^{\prime} \geq(2-\varepsilon) \log \left(\lambda_{1}^{-1}\right)
$$

providing that $\lambda_{1} \leq 0.2$, and $q$ is sufficiently large. Moreover

$$
\lambda^{\prime} \geq \max \left(\frac{3}{2} \log \left(\lambda_{1}^{-1}\right), 1.294\right)
$$

for all sufficiently large $q$ and any $\lambda_{1}$.

We turn now to $\lambda_{2}$, and we suppose that $\lambda_{2} \leq \lambda^{\prime}$, for otherwise information about $\lambda_{2}$ can be read off from Lemma 8.4 and from Tables 2,3 and 4. Again we consider three ranges for $\lambda_{1}$, using methods analogous to the ones applied 


\begin{tabular}{lcccl}
\multicolumn{1}{c}{$\lambda_{1}$} & $\lambda^{\prime}$ & $a$ & $K$ & $\frac{3}{2} \log 1 / \lambda_{1}$ \\
\hline 0.3 & 2.293 & 3.9 & 0.89 & 1.80 \\
0.35 & 2.195 & 4.0 & 0.88 & 1.57 \\
0.4 & 2.108 & 4.1 & 0.88 & 1.37 \\
0.45 & 2.030 & 4.2 & 0.87 & 1.19 \\
0.5 & 1.958 & 4.3 & 0.87 & 1.03 \\
0.55 & 1.893 & 4.4 & 0.86 & 0.89 \\
0.6 & 1.832 & 4.4 & 0.86 & 0.76 \\
0.65 & 1.776 & 4.5 & 0.85 & 0.64 \\
0.7 & 1.724 & 4.5 & 0.85 & \\
0.75 & 1.676 & 4.6 & 0.84 & \\
0.8 & 1.630 & 4.6 & 0.84 & \\
0.85 & 1.587 & 4.7 & 0.84 & \\
0.9 & 1.547 & 4.7 & 0.84 & \\
0.95 & 1.509 & 4.8 & 0.83 & \\
1.0 & 1.473 & 4.8 & 0.83 & \\
1.05 & 1.439 & 4.8 & 0.83 & \\
1.1 & 1.406 & 4.9 & 0.83 & \\
1.15 & 1.375 & 4.9 & 0.83 & \\
1.175 & 1.360 & 4.9 & 0.83 & \\
1.2 & 1.346 & 4.95 & 0.83 & \\
1.225 & 1.331 & 5.0 & 0.83 & \\
1.25 & 1.318 & 5.0 & 0.83 & \\
1.275 & 1.304 & 5.0 & 0.83 & \\
1.294 & 1.294 & 5.0 & 0.83 &
\end{tabular}

Table 4: $\lambda^{\prime}$ for real $\chi_{1}$ and $\rho_{1}$ 
in estimating $\lambda^{\prime}$. We begin by modifying the proof of Lemma 6.2 by replacing $\beta_{1}$ in (6.4) by $\beta_{2}$. The only relevant zero which can be to the right of $\Re(s)=\beta_{2}$ is $\rho_{1}$ itself, since $\lambda^{\prime} \geq \lambda_{2}$, and $\rho_{1}$ occurs for none of the characters $\chi_{2}, \chi_{1} \chi_{2}$ or $\chi_{1} \overline{\chi_{2}}$. Moreover (6.10) may be replaced by $\psi \leq \frac{11}{24}$, since $\phi\left(\chi_{1}\right)=\frac{1}{4}$. We now have

Lemma 8.5 Let $\chi_{1}$ and $\rho_{1}$ be real, and suppose that $\lambda_{2} \leq \lambda^{\prime}$. Then

$$
F\left(-\lambda_{2}\right)-F\left(\lambda_{1}-\lambda_{2}\right)-F(0)+f(0)\left(\frac{11}{24}+\varepsilon\right) \geq 0
$$

for any $\varepsilon>0$, providing that $q$ is sufficiently large.

We can now argue exactly as in the proof of Lemma 8.1 to obtain

$$
\lambda_{1} \geq \frac{\lambda_{2}}{2 e} \exp \left(-\left(\frac{11}{12}+2 \varepsilon\right) \lambda_{2}\right) \geq \exp \left(-\left(\frac{11}{12}+2 \varepsilon\right) \lambda_{2}\right)
$$

for $\lambda_{2} \geq 2 e$ and $q \geq q(\varepsilon)$. Hence we have:

Lemma 8.6 Let $\chi_{1}$ and $\rho_{1}$ be real, and suppose that $\lambda_{2} \leq \lambda^{\prime}$. If $\varepsilon>0$ then either $\lambda_{2}<2 e$ or

$$
\lambda_{2} \geq\left(\frac{12}{11}-\varepsilon\right) \log \left(\lambda_{1}^{-1}\right),
$$

providing that $q$ is sufficiently large.

For the second range of $\lambda_{1}$ we again apply Lemma 8.5, but this time we use the function of Lemmas 7.1 and 7.5 corresponding to $k=2$. We then compute the values in Table 5 in the same way as we did for Tables 1 and 2. We may observe in Table 5 that the condition $\lambda_{2} \leq \lambda^{\prime}$ is redundant, as a comparison with Table 2 shows.

For $\lambda_{1} \geq 0.16$ we shall use our third method. However this will require $\chi_{2}^{4} \neq \chi_{0}$. For those $\chi_{2}$ for which $\chi_{2}^{4}=\chi_{0}$ we shall merely use Lemma 8.5, but with (6.10) replaced by $\psi \leq \frac{3}{8}$. This gives us Table 6 .

We turn now to our third method, in which we assume that $\lambda_{2} \leq \lambda^{\prime}$ and $\chi_{2}^{4} \neq \chi_{0}$. We mimic the argument leading to Lemma 8.2, but starting with the inequality

$$
0 \leq \chi_{0}(n)\left(1+\chi_{1}(n)\right)\left(k+\Re\left\{\chi_{2}(n) n^{-i \gamma_{2}}\right\}\right)^{2},
$$

and using Lemmas 5.2 and 5.3. For any constant $k$ we find that

$$
\begin{aligned}
0 \leq\left(k^{2}+\frac{1}{2}\right)\{ & \left.K\left(\beta_{2}, \chi_{0}\right)+K\left(\beta_{2}, \chi_{1}\right)\right\} \\
& +2 k\left\{K\left(\beta_{2}+i \gamma_{2}, \chi_{2}\right)+K\left(\beta_{2}+i \gamma_{2}, \chi_{1} \chi_{2}\right)\right\} \\
+ & \frac{1}{2}\left\{K\left(\beta_{2}+2 i \gamma_{2}, \chi_{2}^{2}\right)+K\left(\beta_{2}+2 i \gamma_{2}, \chi_{1} \chi_{2}^{2}\right)\right\}
\end{aligned}
$$




\begin{tabular}{lccl}
\multicolumn{1}{c}{$\lambda_{1}$} & $\lambda$ & $\lambda_{2}$ & $\frac{12}{11} \log 1 / \lambda_{1}$ \\
\hline 0.010 & 1.00 & 5.68 & 5.023 \\
0.015 & 0.98 & 5.18 & 4.581 \\
0.020 & 0.97 & 4.83 & 4.267 \\
0.03 & 0.96 & 4.33 & 3.825 \\
0.04 & 0.94 & 3.96 & 3.511 \\
0.05 & 0.93 & 3.67 & 3.268 \\
0.06 & 0.92 & 3.44 & 3.069 \\
0.08 & 0.90 & 3.08 & 2.755 \\
0.10 & 0.89 & 2.83 & 2.511 \\
0.12 & 0.88 & 2.612 & 2.313 \\
0.14 & 0.86 & 2.421 & 2.144 \\
0.16 & 0.85 & 2.257 & 1.999 \\
0.18 & 0.84 & 2.111 & 1.870 \\
0.20 & 0.83 & 1.985 & 1.755
\end{tabular}

Table 5: $\lambda_{2}$ for real $\chi_{1}$ and $\rho_{1}$

\begin{tabular}{lll}
\multicolumn{1}{c}{$\lambda_{1}$} & \multicolumn{1}{c}{$\lambda$} & \multicolumn{1}{c}{$\lambda_{2}$} \\
\hline 0.2 & 1.04 & 2.73 \\
0.3 & 1.00 & 2.13 \\
0.4 & 0.95 & 1.72 \\
0.5 & 0.91 & 1.41 \\
0.6 & 0.88 & 1.17 \\
0.7 & 0.84 & 0.98 \\
0.8 & 0.81 & 0.82 \\
0.809 & 0.809 & 0.809
\end{tabular}

Table 6: $\lambda_{2}$ for real $\chi_{1}$ and $\rho_{1}$ 
and we conclude in the usual way that

$$
\left(k^{2}+\frac{1}{2}\right)\left\{F\left(-\lambda_{2}\right)-F\left(\lambda_{1}-\lambda_{2}\right)\right\}-2 k F(0)+f(0)(\psi+\varepsilon) \geq 0
$$

where

$$
\begin{aligned}
\psi & =\left(k^{2}+\frac{1}{2}\right) \frac{\phi\left(\chi_{1}\right)}{2}+2 k\left(\frac{\phi\left(\chi_{2}\right)}{2}+\frac{\phi\left(\chi_{1} \chi_{2}\right)}{2}\right)+\frac{1}{2}\left(\frac{\phi\left(\chi_{2}^{2}\right)}{2}+\frac{\phi\left(\chi_{1} \chi_{2}^{2}\right)}{2}\right) \\
& \leq \frac{1}{8}\left(k^{2}+\frac{1}{2}\right)+\frac{1}{6}(4 k+1) .
\end{aligned}
$$

It is here that we use our assumptions that $\lambda_{2} \leq \lambda^{\prime}$ and $\chi_{2}^{4} \neq \chi_{0}$. The latter shows that none of the characters $\chi_{2}, \chi_{1} \chi_{2}, \chi_{2}^{2}$ or $\chi_{1} \chi_{2}^{2}$ can be equal to $\chi_{0}$ or $\chi_{1}$. We may therefore state:

Lemma 8.7 Let $\chi_{1}$ and $\rho_{1}$ be real. Assume $\chi_{2}^{4} \neq \chi_{0}$, and that $\lambda_{2} \leq \lambda^{\prime}$. Then for any constant $k \geq 0$, and any $\varepsilon>0$, we have

$$
\left(k^{2}+\frac{1}{2}\right)\left\{F\left(-\lambda_{2}\right)-F\left(\lambda_{1}-\lambda_{2}\right)\right\}-2 k F(0)+f(0)(\psi+\varepsilon) \geq 0
$$

with

$$
\psi=\frac{1}{8}\left(k^{2}+\frac{1}{2}\right)+\frac{1}{6}(4 k+1),
$$

providing that $q \geq q(k, \varepsilon)$.

Since the left hand side of (8.11) is increasing with respect to both $\lambda_{1}$ and $\lambda_{2}$ we may tabulate bounds for $\lambda_{2}$ in the usual way. That is to say, if $\lambda_{1}$ lies in an interval $A \leq \lambda_{1} \leq B$, we choose $k$ and the parameters $\theta, \lambda$ defining $f$ and $F$ in Lemma 7.1, so as to be optimal at $\lambda_{1}=B$. We may then compute a bound for $\lambda_{2}$ for $A \leq \lambda_{1} \leq B$, by using the value $\lambda_{1}=B$ in (8.11). It turns out that the values

$$
k=0.98-0.15 \lambda_{1}
$$

and $\theta=1$ are close to optimal for the relevant range of $\lambda_{1}$, and with these choices we obtain Table 7 . We remark that, when $\lambda_{1}=\lambda_{2}$, the analysis of $\S 7$ applies, so that $\lambda$ and $\lambda_{2}$ should be equal, and $\theta$ should be chosen appropriately. None the less the values we have used in practice give results surprisingly close to those arising from this theoretically optimal choice. Such remarks will also apply in the next section in connection with Table 9.

The condition $\lambda_{2} \leq \lambda^{\prime}$, required for Table 7 , is in fact redundant, as one sees on comparing with Tables 2,3 and 4 . We also observe that if $\chi_{2}^{4}=\chi_{0}$ we get superior bounds for $\lambda_{2}$, as detailed in Table 6, than those listed in Table 7. Thus the values in Table 7 apply whether $\chi_{2}^{4} \neq \chi_{0}$ or not. We note that (8.9) holds in all cases, since $\lambda_{2} \geq \lambda_{1}$, by definition. Finally we always have $\lambda_{2} \geq 0.745$. We summarize as follows. 


\begin{tabular}{cccl}
$\lambda_{1}$ & $\lambda$ & $\lambda_{2}$ & $\frac{12}{11} \log 1 / \lambda_{1}$ \\
\hline 0.10 & 0.79 & 2.76 & 2.511 \\
0.12 & 0.79 & 2.56 & 2.313 \\
0.14 & 0.77 & 2.39 & 2.144 \\
0.16 & 0.77 & 2.25 & 1.999 \\
0.18 & 0.75 & 2.12 & 1.870 \\
0.20 & 0.75 & 2.01 & 1.755 \\
0.25 & 0.73 & 1.77 & 1.512 \\
0.30 & 0.71 & 1.58 & 1.313 \\
0.35 & 0.69 & 1.42 & 1.145 \\
0.40 & 0.68 & 1.29 & 0.999 \\
0.45 & 0.66 & 1.18 & 0.871 \\
0.50 & 0.65 & 1.08 & 0.756 \\
0.55 & 0.64 & 1.00 & 0.652 \\
0.60 & 0.63 & 0.92 & 0.557 \\
0.65 & 0.61 & 0.85 & \\
0.70 & 0.61 & 0.79 & \\
0.745 & 0.59 & 0.745 &
\end{tabular}

Table 7: $\lambda_{2}$ for real $\chi_{1}$ and $\rho_{1}$

Lemma 8.8 Let $\chi_{1}$ and $\rho_{1}$ be real, and let $\varepsilon>0$. Then

$$
\lambda_{2} \geq\left(\frac{12}{11}-\varepsilon\right) \log \left(\lambda_{1}^{-1}\right),
$$

if $q \geq q(\varepsilon)$. Moreover $\lambda_{2} \geq 0.745$ for all sufficiently large $q$.

Lastly, we point out that the estimates of this section have not been proved for extremely small values of $\lambda_{1}$, since we are only concerned with zeros $\rho^{\prime}, \rho_{2}$ in the rectangle (6.1). 


\section{Further Bounds for $\lambda_{1}, \lambda^{\prime}$ and $\lambda_{2}$}

In this section we establish bounds for $\lambda^{\prime}$ and $\lambda_{2}$ in terms of $\lambda_{1}$, in the case in which either $\chi_{1}$ or $\rho_{1}$ is complex. We then use this information to give a lower bound for $\lambda_{1}$.

We begin by considering $\lambda^{\prime}$. We base our estimates on the inequality

$$
\begin{aligned}
& 0 \leq \chi_{0}(n)(1\left.+\Re\left\{\frac{\chi_{1}(n)}{n^{i \gamma^{\prime}}}\right\}\right)\left(K+\Re\left\{\frac{\chi_{1}(n)}{n^{i \gamma_{1}}}\right\}\right)^{2} \\
&=\left(K^{2}+\frac{1}{2}\right)\left(\chi_{0}(n)+\Re\left\{\frac{\chi_{1}(n)}{n^{i \gamma^{\prime}}}\right\}\right) \\
&+ K\left(\Re\left\{\frac{\chi_{0}(n)}{n^{i\left(\gamma_{1}-\gamma^{\prime}\right)}}\right\}+2 \Re\left\{\frac{\chi_{1}(n)}{n^{i \gamma_{1}}}\right\}+\Re\left\{\frac{\chi_{1}(n)^{2}}{n^{i\left(\gamma_{1}+\gamma^{\prime}\right)}}\right\}\right) \\
&+\frac{1}{4}\left(\Re\left\{\frac{\chi_{1}(n)}{n^{i\left(2 \gamma_{1}-\gamma^{\prime}\right)}}\right\}+2 \Re\left\{\frac{\chi_{1}(n)^{2}}{n^{2 i \gamma_{1}}}\right\}+\Re\left\{\frac{\chi_{1}(n)^{3}}{n^{i\left(2 \gamma_{1}+\gamma^{\prime}\right)}}\right\}\right),
\end{aligned}
$$

where $K \geq 0$ is a constant to be chosen. In the notation (8.4), with $\sigma=1+\mathcal{L}^{-1} a$, we then have

$$
\begin{aligned}
0 \leq\left(K^{2}+\frac{1}{2}\right. & \left\{\Sigma\left(\sigma, \chi_{0}\right)+\Sigma\left(\sigma+i \gamma^{\prime}, \chi_{1}\right)\right\} \\
+ & K \Sigma\left(\sigma+i\left(\gamma_{1}-\gamma^{\prime}\right), \chi_{0}\right)+2 K \Sigma\left(\sigma+i \gamma_{1}, \chi_{1}\right) \\
+ & K \Sigma\left(\sigma+i\left(\gamma_{1}+\gamma^{\prime}\right), \chi_{1}^{2}\right)+\frac{1}{4} \Sigma\left(\sigma+i\left(2 \gamma_{1}-\gamma^{\prime}\right), \chi_{1}\right) \\
+ & \frac{1}{2} \Sigma\left(\sigma+2 i \gamma_{1}, \chi_{1}^{2}\right)+\frac{1}{4} \Sigma\left(\sigma+i\left(2 \gamma_{1}+\gamma^{\prime}\right), \chi_{1}^{3}\right)
\end{aligned}
$$

We shall take the polynomial $p(X)$ to be $P_{3}(X)$. We proceed to consider the above terms individually. We begin by assuming that $\chi_{1}$ does not have order 2 or 3 . On writing $\Delta=\mu_{1}-\mu^{\prime}$, we find, in the usual way, that

$$
\begin{aligned}
& \Sigma\left(\sigma, \chi_{0}\right) \leq P_{3}\left(\frac{a+\lambda_{1}}{a}\right)+\varepsilon, \\
& \Sigma\left(\sigma+i \gamma^{\prime}, \chi_{1}\right) \leq-P_{3}\left(\frac{a+\lambda_{1}}{a+\lambda^{\prime}}\right)-\Re\left\{P_{3}\left(\frac{a+\lambda_{1}}{a+\lambda_{1}+i \Delta}\right)\right\} \\
& \Sigma\left(\sigma+i\left(\gamma_{1}-\gamma^{\prime}\right), \chi_{0}\right) \leq\left.\Re\left\{P_{3}\left(\frac{a+\lambda_{1}}{a+i \Delta}\right)\right\}+\varepsilon, \frac{\phi}{2}+\varepsilon\right), \\
& \Sigma\left(\sigma+i \gamma_{1}, \chi_{1}\right) \leq-P_{3}(1)-\Re\left\{P_{3}\left(\frac{a+\lambda_{1}}{a+\lambda^{\prime}+i \Delta}\right)\right\} \\
&+\left(a+\lambda_{1}\right)\left(\frac{\phi}{2}+\varepsilon\right), \\
& \Sigma\left(\sigma+i\left(\gamma_{1}+\gamma^{\prime}\right), \chi_{1}^{2}\right) \leq\left(a+\lambda_{1}\right)\left(\frac{\phi}{2}+\varepsilon\right),
\end{aligned}
$$




$$
\begin{aligned}
\Sigma\left(\sigma+i\left(2 \gamma_{1}-\gamma^{\prime}\right), \chi_{1}\right) & \leq-\Re\left\{P_{3}\left(\frac{a+\lambda_{1}}{a+\lambda_{1}+i \Delta}\right)\right\}+\left(a+\lambda_{1}\right)\left(\frac{\phi}{2}+\varepsilon\right),(9.5 \\
\Sigma\left(\sigma+2 i \gamma_{1}, \chi_{1}^{2}\right) & \leq\left(a+\lambda_{1}\right)\left(\frac{\phi}{2}+\varepsilon\right)
\end{aligned}
$$

and

$$
\Sigma\left(\sigma+i\left(2 \gamma_{1}+\gamma^{\prime}\right), \chi_{1}^{3}\right) \leq\left(a+\lambda_{1}\right)\left(\frac{\phi}{2}+\varepsilon\right) .
$$

On feeding these bounds into (9.1) we obtain

$$
0 \leq\left(K^{2}+\frac{1}{2}\right)\left\{P_{3}\left(\frac{a+\lambda_{1}}{a}\right)-P_{3}\left(\frac{a+\lambda_{1}}{a+\lambda^{\prime}}\right)\right\}-2 K P_{3}(1)-A+\left(a+\lambda_{1}\right)(\psi+\varepsilon),
$$

where

$$
A=\Re\left\{\left(K^{2}+\frac{3}{4}\right) P_{3}\left(\frac{a+\lambda_{1}}{a+\lambda_{1}+i \Delta}\right)+2 K P_{3}\left(\frac{a+\lambda_{1}}{a+\lambda^{\prime}+i \Delta}\right)-K P_{3}\left(\frac{a+\lambda_{1}}{a+i \Delta}\right)\right\}
$$

and

$$
\psi=\left(K^{2}+3 K+\frac{3}{2}\right) \frac{\phi}{2} .
$$

We handle $A$ just as in the proof of Lemma 8.3, and we find that $A \geq 0$ providing that

$$
\frac{K^{2}+\frac{3}{4}}{\left(a+\lambda_{1}\right)^{3}}+\frac{2 K}{\left(a+\lambda^{\prime}\right)^{3}} \geq \frac{K}{a^{3}} .
$$

We now turn to the case in which $\chi_{1}^{3}=\chi_{0}$. Here we replace (9.7) by

$$
\Sigma\left(\sigma+i\left(2 \gamma_{1}+\gamma^{\prime}\right), \chi_{1}^{3}\right) \leq \Re\left\{P_{3}\left(\frac{a+\lambda_{1}}{a+i \alpha}\right)\right\}+\varepsilon,
$$

where $\alpha=2 \mu_{1}+\mu^{\prime}$. By way of compensation, the bounds (9.4) and (9.6) may be sharpened to

$$
\Sigma\left(\sigma+i\left(\gamma_{1}+\gamma^{\prime}\right), \chi_{1}^{2}\right) \leq-\Re\left\{P_{3}\left(\frac{a+\lambda_{1}}{a+\lambda_{1}+i \alpha}\right)\right\}+\left(a+\lambda_{1}\right)\left(\frac{\phi}{2}+\varepsilon\right),
$$

and

$$
\Sigma\left(\sigma+2 i \gamma_{1}, \chi_{1}^{2}\right) \leq-\Re\left\{P_{3}\left(\frac{a+\lambda_{1}}{a+\lambda^{\prime}+i \alpha}\right)\right\}+\left(a+\lambda_{1}\right)\left(\frac{\phi}{2}+\varepsilon\right),
$$

respectively. The overall effect is to replace $A$ in (9.8) by $A+B$, where

$$
B=\Re\left\{K P_{3}\left(\frac{a+\lambda_{1}}{a+\lambda_{1}+i \alpha}\right)+\frac{1}{2} \Re\left\{P_{3}\left(\frac{a+\lambda_{1}}{a+\lambda^{\prime}+i \alpha}\right)\right\}-\frac{1}{4} \Re\left\{P_{3}\left(\frac{a+\lambda_{1}}{a+i \alpha}\right)\right\},\right.
$$

and to reduce $\psi$ to

$$
\psi=\left(K^{2}+3 K+\frac{5}{4}\right) \frac{\phi}{2} .
$$


In order to ensure that $B \geq 0$ it merely suffices that

$$
\frac{K}{\left(a+\lambda_{1}\right)^{3}}+\frac{1 / 2}{\left(a+\lambda^{\prime}\right)^{3}} \geq \frac{1 / 4}{a^{3}}
$$

by an argument analogous to that used for the proof of Lemma 8.3.

Finally we consider the case in which $\chi_{1}^{2}=\chi_{0}$. Here we may use three distinct zeros $\rho_{1}, \overline{\rho_{1}}$ and $\rho^{\prime}$ of $L\left(s, \chi_{1}\right)$. However we shall not assume that $\rho^{\prime}$ is complex. In the present case (9.4) and (9.6) must be replaced by

$$
\Sigma\left(\sigma+i\left(\gamma_{1}+\gamma^{\prime}\right), \chi_{1}^{2}\right) \leq \Re\left\{P_{3}\left(\frac{a+\lambda_{1}}{a+i \beta}\right)\right\}+\varepsilon,
$$

and

$$
\Sigma\left(\sigma+2 i \gamma_{1}, \chi_{1}^{2}\right) \leq \Re\left\{P_{3}\left(\frac{a+\lambda_{1}}{a+i \gamma}\right)\right\}+\varepsilon,
$$

respectively, where $\beta=\mu_{1}+\mu^{\prime}$, and $\gamma=2 \mu_{1}$. On the other hand we may improve (9.2),(9.5) and (9.7) to

$$
\begin{gathered}
\Sigma\left(\sigma+i \gamma^{\prime}, \chi_{1}\right) \leq-P_{3}\left(\frac{a+\lambda_{1}}{a+\lambda^{\prime}}\right)-\Re\left\{P_{3}\left(\frac{a+\lambda_{1}}{a+\lambda_{1}+i \Delta}\right)\right\} \\
-\Re\left\{P_{3}\left(\frac{a+\lambda_{1}}{a+\lambda_{1}+i \beta}\right)\right\}+\left(a+\lambda_{1}\right)\left(\frac{\phi}{2}+\varepsilon\right), \\
\Sigma\left(\sigma+i\left(2 \gamma_{1}-\gamma^{\prime}\right), \chi_{1}\right) \leq-\Re\left\{P_{3}\left(\frac{a+\lambda_{1}}{a+\lambda_{1}+i \Delta}\right)\right\}-\Re\left\{P_{3}\left(\frac{a+\lambda_{1}}{a+\lambda^{\prime}+i \gamma}\right)\right\} \\
+\left(a+\lambda_{1}\right)\left(\frac{\phi}{2}+\varepsilon\right),
\end{gathered}
$$

and

$$
\begin{aligned}
\Sigma\left(\sigma+i\left(2 \gamma_{1}+\gamma^{\prime}\right), \chi_{1}^{3}\right) \leq-\Re\left\{P_{3}\left(\frac{a+\lambda_{1}}{a+\lambda_{1}+i \beta}\right)\right\} & -\Re\left\{P_{3}\left(\frac{a+\lambda_{1}}{a+\lambda^{\prime}+i \gamma}\right)\right\} \\
& +\left(a+\lambda_{1}\right)\left(\frac{\phi}{2}+\varepsilon\right),
\end{aligned}
$$

The estimate $(9.3)$ requires a little more care. If $\gamma^{\prime} \neq 0$ we find

$$
\begin{aligned}
\Sigma\left(\sigma+i \gamma_{1}, \chi_{1}\right) \leq-P_{3}(1) & -\Re\left\{P_{3}\left(\frac{a+\lambda_{1}}{a+\lambda^{\prime}+i \Delta}\right)\right\}-\Re\left\{P_{3}\left(\frac{a+\lambda_{1}}{a+\lambda^{\prime}+i \beta}\right)\right\} \\
& -\Re\left\{P_{3}\left(\frac{a+\lambda_{1}}{a+\lambda_{1}+i \gamma}\right)\right\}+\left(a+\lambda_{1}\right)\left(\frac{\phi}{2}+\varepsilon\right) .
\end{aligned}
$$

However if $\gamma^{\prime}=0$ we obtain only the weaker bound 


$$
\begin{array}{r}
\Sigma\left(\sigma+i \gamma_{1}, \chi_{1}\right) \\
\leq-P_{3}(1)-\Re\left\{P_{3}\left(\frac{a+\lambda_{1}}{a+\lambda^{\prime}+i \mu_{1}}\right)\right\}-\Re\left\{P_{3}\left(\frac{a+\lambda_{1}}{a+\lambda_{1}+i \gamma}\right)\right\} \\
+\left(a+\lambda_{1}\right)\left(\frac{\phi}{2}+\varepsilon\right) . \\
=-P_{3}(1)-\frac{1}{2} \Re\left\{P_{3}\left(\frac{a+\lambda_{1}}{a+\lambda^{\prime}+i \Delta}\right)\right\}-\frac{1}{2} \Re\left\{P_{3}\left(\frac{a+\lambda_{1}}{a+\lambda^{\prime}+i \beta}\right)\right\} \\
-\Re\left\{P_{3}\left(\frac{a+\lambda_{1}}{a+\lambda_{1}+i \gamma}\right)\right\}+\left(a+\lambda_{1}\right)\left(\frac{\phi}{2}+\varepsilon\right) .
\end{array}
$$

The overall effect of these results is to replace $A$ in (9.8) by $A^{\prime}+C+D$, where

$$
\begin{aligned}
A^{\prime} & =\Re\left\{\left(K^{2}+\frac{3}{4}\right) P_{3}\left(\frac{a+\lambda_{1}}{a+\lambda_{1}+i \Delta}\right)+K P_{3}\left(\frac{a+\lambda_{1}}{a+\lambda^{\prime}+i \Delta}\right)-K P_{3}\left(\frac{a+\lambda_{1}}{a+i \Delta}\right)\right\}, \\
C & =\Re\left\{\left(K^{2}+\frac{3}{4}\right) P_{3}\left(\frac{a+\lambda_{1}}{a+\lambda_{1}+i \beta}\right)+K P_{3}\left(\frac{a+\lambda_{1}}{a+\lambda^{\prime}+i \beta}\right)-K P_{3}\left(\frac{a+\lambda_{1}}{a+i \beta}\right)\right\},
\end{aligned}
$$

and

$$
D=\Re\left\{2 K P_{3}\left(\frac{a+\lambda_{1}}{a+\lambda_{1}+i \gamma}\right)+\frac{1}{2} P_{3}\left(\frac{a+\lambda_{1}}{a+\lambda^{\prime}+i \gamma}\right)-\frac{1}{2} P_{3}\left(\frac{a+\lambda_{1}}{a+i \gamma}\right)\right\},
$$

and to reduce $\psi$ to

$$
\psi=\left(K^{2}+2 K+1\right) \frac{\phi}{2} .
$$

As before, we see that $A^{\prime}, C, D \geq 0$, providing that

$$
\frac{K^{2}+\frac{3}{4}}{\left(a+\lambda_{1}\right)^{3}}+\frac{K}{\left(a+\lambda^{\prime}\right)^{3}} \geq \frac{K}{a^{3}}
$$

and

$$
\frac{2 K}{\left(a+\lambda_{1}\right)^{3}}+\frac{1 / 2}{\left(a+\lambda^{\prime}\right)^{3}} \geq \frac{1 / 2}{a^{3}} .
$$

If we examine the conditions $(9.9),(9.10),(9.11)$ and (9.12) we find that the last two are the most stringent. We may therefore summarize as follows.

Lemma 9.1 Let $\chi_{1}$ or $\rho_{1}$ (or both) be complex. Let $a, K$ and $\varepsilon$ be positive constants. Then

$$
\begin{gathered}
\left(K^{2}+\frac{1}{2}\right)\left\{P_{3}\left(\frac{a+\lambda_{1}}{a}\right)-P_{3}\left(\frac{a+\lambda_{1}}{a+\lambda^{\prime}}\right)\right\}-2 K P_{3}(1)+\left(a+\lambda_{1}\right)(\psi+\varepsilon) \\
\geq 0,
\end{gathered}
$$

where

$$
\psi=\left(K^{2}+3 K+\frac{3}{2}\right) \frac{\phi}{2}
$$


for sufficiently large $q$, providing that

$$
K_{0}\left(a+\lambda_{1}\right)^{-3}+\left(a+\lambda^{\prime}\right)^{-3} \geq a^{-3},
$$

with

$$
K_{0}=\min \left(K+\frac{3}{4 K}, 4 K\right) .
$$

Of course we may improve the value of $\psi$ when $\chi_{1}$ has order 2 or 3 , but the result quoted will suffice for our purposes. Since the right hand side of (9.13) is increasing with respect to both $\lambda_{1}$ and $\lambda^{\prime}$, we may tabulate values in the usual way. The choice

$$
K=0.775+0.15 \lambda_{1}
$$

is roughly optimal, and with this we produce Table 8 .

We are now ready to consider estimates for $\lambda_{2}$. The methods we shall use are related to those of $\S 8$ We start with an analogue of Lemma 8.7, which will indeed be of interest for $\lambda_{3}$ also. Taking $j=2$ or 3 , we begin with the inequality

$$
\begin{aligned}
0 \leq \chi_{0}(n)\left(1+\Re\left\{\frac{\chi_{j}(n)}{n^{i \gamma_{j}}}\right\}\right)\left(k+\Re\left\{\frac{\chi_{1}(n)}{n^{i \gamma_{1}}}\right\}\right)^{2} \\
=\left(k^{2}+\frac{1}{2}\right)\left[\chi_{0}(n)+\Re\left\{\frac{\chi_{j}(n)}{n^{i \gamma_{j}}}\right\}\right]+k \Re\left\{\frac{\chi_{1} \chi_{j}(n)}{n^{i\left(\gamma_{1}+\gamma_{j}\right)}}\right\} \\
+2 k \Re\left\{\frac{\chi_{1}(n)}{n^{i \gamma_{1}}}\right\}+k \Re\left\{\frac{\overline{\chi_{1}} \chi_{j}(n)}{n^{i\left(-\gamma_{1}+\gamma_{j}\right)}}\right\}+\frac{1}{4} \Re\left\{\frac{\chi_{1}^{2} \chi_{j}(n)}{n^{i\left(2 \gamma_{1}+\gamma_{j}\right)}}\right\} \\
+\frac{1}{2} \Re\left\{\frac{\chi_{1}^{2}(n)}{n^{2 i \gamma_{1}}}\right\}++\frac{1}{4} \Re\left\{\frac{\bar{\chi}_{1}^{2} \chi_{j}(n)}{n^{i\left(-2 \gamma_{1}+\gamma_{j}\right)}}\right\},
\end{aligned}
$$

whence

$$
\begin{gathered}
0 \leq\left(k^{2}+\frac{1}{2}\right)\left\{K\left(\beta_{1}, \chi_{0}\right)+K\left(\beta_{1}+i \gamma_{j}, \chi_{j}\right)\right\}+k K\left(\beta_{1}+i\left(\gamma_{1}+\gamma_{j}\right), \chi_{1} \chi_{j}\right) \\
+2 k K\left(\beta_{1}+i \gamma_{1}, \chi_{1}\right)+k K\left(\beta_{1}+i\left(-\gamma_{1}+\gamma_{j}\right), \overline{\chi_{1}} \chi_{j}\right) \\
+\frac{1}{4} K\left(\beta_{1}+i\left(2 \gamma_{1}+\gamma_{j}\right), \chi_{1}^{2} \chi_{j}\right)+\frac{1}{2} K\left(\beta_{1}+2 i \gamma_{1}, \chi_{1}^{2}\right) \\
+\frac{1}{4} K\left(\beta_{1}+i\left(-2 \gamma_{1}+\gamma_{j}\right),{\overline{\chi_{1}}}^{2} \chi_{j}\right) .
\end{gathered}
$$

Thus, if none of the characters $\chi_{1} \chi_{j}, \overline{\chi_{1}} \chi_{j}, \chi_{1}^{2} \chi_{j}, \chi_{1}^{2}$ or ${\overline{\chi_{1}}}^{2} \chi_{j}$ is principal, we deduce that

$$
\left(k^{2}+\frac{1}{2}\right)\left\{F\left(-\lambda_{1}\right)-F\left(\lambda_{j}-\lambda_{1}\right)\right\}-2 k F(0)+f(0)\{\psi+\varepsilon\} \geq 0,
$$

with

$$
\psi \leq\left(k^{2}+4 k+\frac{3}{2}\right) \frac{\phi}{2}
$$




\begin{tabular}{ccc}
$\lambda_{1}$ & $\lambda^{\prime}$ & $a$ \\
\hline 0.30 & 1.526 & 2.18 \\
0.32 & 1.446 & 2.23 \\
0.34 & 1.374 & 2.28 \\
0.36 & 1.309 & 2.32 \\
0.38 & 1.250 & 2.36 \\
0.40 & 1.196 & 2.40 \\
0.42 & 1.145 & 2.43 \\
0.44 & 1.099 & 2.46 \\
0.46 & 1.055 & 2.49 \\
0.48 & 1.015 & 2.51 \\
0.50 & 0.977 & 2.53 \\
0.52 & 0.941 & 2.55 \\
0.54 & 0.907 & 2.57 \\
0.56 & 0.875 & 2.59 \\
0.58 & 0.845 & 2.61 \\
0.60 & 0.816 & 2.63 \\
0.62 & 0.789 & 2.64 \\
0.64 & 0.763 & 2.65 \\
0.66 & 0.783 & 2.66 \\
0.68 & 0.714 & 2.68 \\
0.696 & 0.696 & 2.69
\end{tabular}

Table 8: $\lambda^{\prime}$ in the complex case 


\begin{tabular}{ccc}
$\lambda_{1}$ & $\lambda_{j}$ & $\lambda$ \\
\hline 0.34 & 1.283 & 0.44 \\
0.36 & 1.230 & 0.45 \\
0.38 & 1.181 & 0.46 \\
0.40 & 1.135 & 0.47 \\
0.42 & 1.094 & 0.48 \\
0.44 & 1.055 & 0.48 \\
0.46 & 1.018 & 0.49 \\
0.48 & 0.984 & 0.50 \\
0.50 & 0.952 & 0.51 \\
0.52 & 0.922 & 0.51 \\
0.54 & 0.893 & 0.52 \\
0.56 & 0.866 & 0.52 \\
0.58 & 0.840 & 0.53 \\
0.60 & 0.816 & 0.53 \\
0.62 & 0.792 & 0.54 \\
0.64 & 0.770 & 0.54 \\
0.66 & 0.748 & 0.55 \\
0.68 & 0.728 & 0.55 \\
0.70 & 0.708 & 0.56 \\
0.704 & 0.704 & 0.56
\end{tabular}

Table 9: $\lambda_{j}$ in the complex case, $\chi_{j} \neq \chi_{1}^{2},{\overline{\chi_{1}}}^{2}$

We therefore obtain:

Lemma 9.2 Let $\varepsilon>0$ be given, and suppose that $j=2$ or 3 . Then if $\chi_{1}^{2} \neq$ $\chi_{0}, \chi_{j}, \overline{\chi_{j}}$, we have

$$
\left(k^{2}+\frac{1}{2}\right)\left\{F\left(-\lambda_{1}\right)-F\left(\lambda_{j}-\lambda_{1}\right)\right\}-2 k F(0)+\frac{f(0)}{6}\left(k^{2}+4 k+\frac{3}{2}+\varepsilon\right) \geq 0
$$

for any constant $k \geq 0$, and any sufficiently large $q$.

Of course the lemma will apply to at least one of $\chi_{2}$ or $\chi_{3}$, unless $\chi_{1}$ is real. Thus for complex $\chi_{1}$, we obtain bounds for $\lambda_{3}$, and often for $\lambda_{2}$. The optimal values for the parameter $\theta$ in Lemma 7.1 and for the constant $k$ in Lemma 7.5 are approximately $\theta=1$ and

$$
k=0.78+0.1 \lambda_{1} .
$$

With these choices a table of values arising from the lemma is given as Table 9 .

When $\chi_{2}=\chi_{1}^{2}$ or $\bar{\chi}_{1}^{2}$ an alternative method is required. We merely reverse the rôles of $\chi_{1}$ and $\chi_{j}$ in the proof of Lemma 9.2 to obtain the following. 
Lemma 9.3 Let positive constants $k$ and $\varepsilon$ be given, and suppose that $\chi_{2}^{2} \neq$ $\chi_{0}, \chi_{1}, \overline{\chi_{1}}$. then

$$
\left(k^{2}+\frac{1}{2}\right)\left\{F\left(-\lambda_{1}\right)-F(0)\right\}-2 k F\left(\lambda_{2}-\lambda_{1}\right)+\frac{f(0)}{6}\left(k^{2}+4 k+\frac{3}{2}+\varepsilon\right) \geq 0
$$

for sufficiently large $q$.

The optimal choices here are roughly $\theta=1$ and

$$
k=0.94-0.1 \lambda_{1} .
$$

The function on the left of (9.14) is obviously increasing with $\lambda_{2}$. However its behaviour with respect to $\lambda_{1}$ is not so clear. In order to tabulate values of $\lambda_{1}$ and $\lambda_{2}$ we therefore observe that if the left hand side is negative at $\left(\lambda_{1}, \lambda_{2}\right)=(a-\delta, b)$ and at $(a, b)$, then it will also be negative at $\left(a^{\prime}, b-\delta\right)$ for $a-\delta \leq a^{\prime} \leq a$. Hence $a-\delta \leq \lambda_{1} \leq a$ will imply $\lambda_{2} \geq b-\delta$. We use this principle, with $\delta=0.002$, to construct Table 10. Thus for example, the line $\lambda_{1}=0.4, \lambda_{2}=0.871, \lambda=0.49$, means that $\lambda_{2} \geq 0.871$ whenever $\lambda_{1} \leq 0.4$. The verification of this involved checking that (9.14) fails for

$$
\left(\lambda_{1}, \lambda_{2}\right)=(0.4,0.873),(0.398,0.873),(0.396,0.873), \ldots,(0.38,0.873) .
$$

Comparison of Tables 9 and 10 shows that the latter gives weaker results. Table 10 therefore applies unless both

$$
\chi_{2}^{2}=\chi_{0}, \chi_{1} \text { or } \overline{\chi_{1}}
$$

and

$$
\chi_{1}^{2}=\chi_{0}, \chi_{2} \text { or } \overline{\chi_{2}} \text {. }
$$

This will happen only when $\chi_{1}$ and $\chi_{2}$ have order 5 or less. To cover this situation we therefore use Lemma 6.2 , with $\psi \leq \frac{3}{8}$. This readily produces Table 11. The bounds for this case are sharper than those in Table 10. Moreover the bounds in Table 7 are sharper than those in Table 10. We may therfore summarize as follows.

Lemma 9.4 The bounds given in Table 10 apply in all cases. In particular we have $\lambda_{2} \geq 0.702$ for all sufficiently large $q$.

We now have the necessary material for the estimation of $\lambda_{1}$. We shall use the inequality

$$
\begin{aligned}
0 & \leq(3+10 \cos \theta)^{2}(9+10 \cos \theta)^{2} \\
& =14379+24480 \cos \theta+14900 \cos 2 \theta+6000 \cos 3 \theta+1250 \cos 4 \theta .
\end{aligned}
$$

This is close to that employed by Chen [6]. It turns out that the above inequality is close to optimal in our situation too. In the usual way we find that

$$
\begin{gathered}
0 \leq 14379 K\left(\beta, \chi_{0}\right)+24480 K\left(\beta+i \gamma_{1}, \chi_{1}\right)+14900 K\left(\beta+2 i \gamma_{1}, \chi_{1}^{2}\right) \\
+6000 K\left(\beta+3 i \gamma_{1}, \chi_{1}^{3}\right)+1250 K\left(\beta+4 i \gamma_{1}, \chi_{1}^{4}\right) .
\end{gathered}
$$




\begin{tabular}{ccc}
$\lambda_{1}$ & $\lambda_{2}$ & $\lambda$ \\
\hline 0.34 & 0.929 & 0.47 \\
0.36 & 0.903 & 0.48 \\
0.38 & 0.887 & 0.48 \\
0.40 & 0.871 & 0.49 \\
0.42 & 0.856 & 0.50 \\
0.44 & 0.842 & 0.50 \\
0.46 & 0.829 & 0.50 \\
0.48 & 0.816 & 0.51 \\
0.50 & 0.803 & 0.52 \\
0.52 & 0.791 & 0.52 \\
0.54 & 0.780 & 0.53 \\
0.56 & 0.769 & 0.53 \\
0.58 & 0.759 & 0.54 \\
0.60 & 0.749 & 0.55 \\
0.62 & 0.739 & 0.55 \\
0.64 & 0.730 & 0.55 \\
0.66 & 0.721 & 0.55 \\
0.68 & 0.712 & 0.55 \\
0.70 & 0.704 & 0.56 \\
0.702 & 0.702 & 0.56
\end{tabular}

Table 10: $\lambda_{2}$ in the complex case

\begin{tabular}{lll}
$\lambda_{1}$ & \multicolumn{1}{c}{$\lambda_{2}$} & \multicolumn{1}{c}{$\lambda$} \\
\hline 0.34 & 1.28 & 0.65 \\
0.36 & 1.25 & 0.66 \\
0.38 & 1.22 & 0.67 \\
0.40 & 1.20 & 0.68 \\
0.50 & 1.07 & 0.72 \\
0.60 & 0.97 & 0.75 \\
0.70 & 0.89 & 0.77 \\
0.808 & 0.808 & 0.81
\end{tabular}

Table 11: $\lambda_{2}$ in the complex case, $\phi=\frac{1}{4}$ 
We shall take $\beta=1-\lambda^{*} / \mathcal{L}$, with a constant $\lambda^{*} \leq \lambda^{\prime}, \lambda_{2}$. If $\chi_{1}$ has order 6 or more, then Lemma 5.2 gives us

$$
K\left(\beta+n i \gamma_{1}, \chi_{1}^{n}\right) \leq f(0)\left(\frac{1}{6}+\varepsilon\right) \mathcal{L} \quad(n=2,3,4),
$$

since $\beta \geq \beta_{2}$, and $\chi_{1}^{n} \neq \chi_{0}, \chi_{1}, \overline{\chi_{1}}$. On the other hand

$$
K\left(\beta+i \gamma_{1}, \chi_{1}\right) \leq-\mathcal{L} F\left(\lambda_{1}-\lambda^{*}\right)+f(0)\left(\frac{1}{6}+\varepsilon\right) \mathcal{L},
$$

since $\beta \geq \beta^{\prime}$. Moreover Lemma 5.3 yields

$$
K\left(\beta, \chi_{0}\right) \leq \mathcal{L} F\left(-\lambda^{*}\right)+f(0) \varepsilon \mathcal{L} .
$$

We deduce that

$$
14379 F\left(-\lambda^{*}\right)-24480 F\left(\lambda_{1}-\lambda^{*}\right)+\frac{46630}{6} f(0)+\varepsilon \geq 0
$$

with a new $\varepsilon$. We shall apply this with the function from Lemma 7.1 corresponding to $\theta=1$ and $\lambda=0.5$. We argue by contradiction. If $\lambda_{1} \leq 0.38$ then Tables 8 and 10 yield $\lambda^{\prime}, \lambda_{2} \geq 0.887$. Taking $\lambda^{*}=0.887$ we would deduce that

$$
14379 F(-0.887)-24480 F\left(\lambda_{1}-0.887\right)+\frac{46630}{6} f(0)+\varepsilon \geq 0
$$

for large enough $q$, whence $\lambda_{1} \geq 0.364$. Thus $\lambda_{1} \geq 0.364$ whether $\lambda_{1} \leq 0.38$ or not.

When $\chi_{1}$ has order $2,3,4$ or 5 we may take $\phi=\frac{1}{4}$ instead of $\phi=\frac{1}{3}$. However there are complications arising from terms $\chi_{1}^{n}=\chi_{0}, \chi_{1}$ or $\overline{\chi_{1}}$. When $\chi_{1}$ has order 5 we use (9.16) with $\beta=\beta_{1}$, giving

$$
14379 F\left(-\lambda_{1}\right)-24480 F(0)+\frac{46630}{8} f(0)+\varepsilon \geq 0 .
$$

The optimisation problem here is exactly that addressed in $\S 7$, with

$$
k=24480 / 14379 .
$$

Choosing $\theta$ as in Lemma 7.5 yields $\theta=1.1580 \ldots$ and

$$
\lambda_{1}^{-1} \cos ^{2} \theta \leq \frac{1}{8} \cdot \frac{46630}{14379}+\varepsilon,
$$

whence $\lambda_{1} \geq 0.397$. When $\chi_{1}$ has order $h=2,3$ or 4 we again use the inequality (9.16), but now in conjunction with the function (8.4) corresponding to the polynomial $P_{3}(X)$. On choosing $s=1+a \mathcal{L}^{-1}$ as before, this leads to

$$
14379 P_{3}\left(\frac{a+\lambda_{1}}{a}\right)-24480 P_{3}(1)+\Re\left\{T_{h}\left(h \gamma_{1}\right)\right\}+N_{h}\left(a+\lambda_{1}\right)\left(\frac{\phi}{2}+\varepsilon\right) \geq 0,
$$


where

$$
\begin{aligned}
T_{2}(t)= & -24480 P_{3}\left(\frac{a+\lambda_{1}}{a+\lambda_{1}+i t}\right)+14900 P_{3}\left(\frac{a+\lambda_{1}}{a+i t}\right)-6000 P_{3}\left(\frac{a+\lambda_{1}}{a+\lambda_{1}+i t}\right) \\
& -6000 P_{3}\left(\frac{a+\lambda_{1}}{a+\lambda_{1}+2 i t}\right)+1250 P_{3}\left(\frac{a+\lambda_{1}}{a+2 i t}\right), \\
T_{3}(t)= & -14900 P_{3}\left(\frac{a+\lambda_{1}}{a+\lambda_{1}+i t}\right)+6000 P_{3}\left(\frac{a+\lambda_{1}}{a+i t}\right)-1250 P_{3}\left(\frac{a+\lambda_{1}}{a+\lambda_{1}+i t}\right), \\
T_{4}(t)= & -6000 P_{3}\left(\frac{a+\lambda_{1}}{a+\lambda_{1}+i t}\right)+1250 P_{3}\left(\frac{a+\lambda_{1}}{a+i t}\right),
\end{aligned}
$$

and

$$
N_{2}=30480, \quad N_{3}=40630, \quad N_{4}=45380 .
$$

Since $\Re\left(P_{3}(\alpha / \alpha+i t)\right) \geq 0$ we see that $\Re T_{h}(t) \leq 0$ providing that

$$
30480 P_{3}\left(\frac{a+\lambda_{1}}{a+\lambda_{1}+i t}\right) \geq 14900 P_{3}\left(\frac{a+\lambda_{1}}{a+i t}\right) .
$$

The argument leading to Lemma 8.3 then shows that it suffices to have

$$
\frac{30480}{\left(a+\lambda_{1}\right)^{3}} \geq \frac{14900}{a^{3}} \text {. }
$$

We conclude that

$$
14379 P_{3}\left(\frac{a+\lambda_{1}}{a}\right)-24480 P_{3}(1)+\frac{1}{8} N_{h}\left(a+\lambda_{1}\right)+\varepsilon \geq 0
$$

providing that (9.17) holds. We apply this with $a=3.4$ for $h=2 ; a=2.6$ for $h=3$; and $a=2.3$ for $h=4$. We then deduce that $\lambda_{1} \geq 0.518, \lambda_{1} \geq 0.389$, and $\lambda_{1} \geq 0.348$ in the three cases.

In summary we now have:

Lemma 9.5 Suppose that either $\chi_{1}$ or $\rho_{1}$ is complex, or both, and let $\chi_{1}$ have order $h$. There is an absolute constant $q_{0}$ such that for $q \geq q_{0}$ we have

$$
\begin{aligned}
& \lambda_{1} \geq 0.518, \quad(h=2), \\
& \lambda_{1} \geq 0.389, \quad(h=3), \\
& \lambda_{1} \geq 0.348, \quad(h=4), \\
& \lambda_{1} \geq 0.397, \quad(h=5),
\end{aligned}
$$

and

$$
\lambda_{1} \geq 0.364, \quad(h \geq 6) .
$$




\section{Bounds for $\lambda_{3}$}

In this section we shall estimate $\lambda_{3}$ from below. We shall argue by contradiction, assuming throughout that $\lambda_{3} \leq 6 / 7$. Our starting point is the inequality

$$
\prod_{j=1}^{3}\left\{1+\Re\left(\chi_{j}(n) n^{-i \gamma_{j}}\right)\right\} \geq 0,
$$

whence

$$
K\left(\beta_{1}, \chi_{0}\right)+\sum_{j=1}^{3} K\left(\beta_{1}+i \gamma_{j}\right)+\frac{1}{2} \Sigma_{2}+\frac{1}{4} \Sigma_{3} \geq 0
$$

with

$$
\Sigma_{2}=\sum_{1 \leq j<k \leq 3}\left\{K\left(\beta_{1}+i \gamma_{j}+i \gamma_{k}, \chi_{j} \chi_{k}\right)+K\left(\beta_{1}+i \gamma_{j}-i \gamma_{k}, \chi_{j} \overline{\chi_{k}}\right)\right\},
$$

and

$$
\begin{gathered}
\Sigma_{3}=K\left(\beta_{1}+i \gamma_{1}+i \gamma_{2}+i \gamma_{3}, \chi_{1} \chi_{2} \chi_{3}\right)+K\left(\beta_{1}+i \gamma_{1}+i \gamma_{2}-i \gamma_{3}, \chi_{1} \chi_{2} \overline{\chi_{3}}\right) \\
+K\left(\beta_{1}+i \gamma_{1}-i \gamma_{2}+i \gamma_{3}, \chi_{1} \overline{\chi_{2}} \chi_{3}\right) \\
+K\left(\beta_{1}+i \gamma_{1}-i \gamma_{2}-i \gamma_{3}, \chi_{1} \overline{\chi_{2} \chi_{3}}\right) .
\end{gathered}
$$

Since $\chi_{j}, \chi_{j} \chi_{k}$ and $\chi_{j} \overline{\chi_{k}}$ are automatically non-principal, it follows in the usual way that

$$
\sum_{j=1}^{3} K\left(\beta_{1}+i \gamma_{j}\right) \leq-\mathcal{L}\left\{F(0)+F\left(\lambda_{2}-\lambda_{1}\right)+F\left(\lambda_{1}-\lambda_{3}\right)\right\}+f(0)\left(\frac{3 \phi}{2}+\varepsilon\right) \mathcal{L}
$$

and

$$
\Sigma_{2} \leq f(0)\left(\frac{6 \phi}{2}+\varepsilon\right) \mathcal{L} .
$$

If moreover none of the characters involved in $\Sigma_{3}$ are principal we will have

$$
\Sigma_{3} \leq f(0)\left(\frac{4 \phi}{2}+\varepsilon\right) \mathcal{L}
$$

and hence

$$
F\left(-\lambda_{1}\right)-F(0)-F\left(\lambda_{2}-\lambda_{1}\right)-F\left(\lambda_{3}-\lambda_{1}\right)+f(0)\left(\frac{7}{6}+2 \varepsilon\right) \geq 0 .
$$

We therefore use the function from Lemma 7.2, and we choose $\lambda=\left(\frac{7}{6}+2 \varepsilon\right)^{-1}$. Then Lemma 7.5 yields

$$
\lambda^{-1} f(0)=3 F(0)-F(-\lambda)
$$


so that either $\lambda_{3}>\lambda$ or

$$
2\left(F(0)-F\left(\lambda-\lambda_{1}\right)\right) \geq F(-\lambda)-F\left(-\lambda_{1}\right),
$$

from (10.6).

One may show numerically that this cannot happen. For $0 \leq \lambda_{1} \leq \lambda$ we have

$$
2\left(F(0)-F\left(\lambda-\lambda_{1}\right)\right) \leq 2\left(\lambda-\lambda_{1}\right)\left|F^{\prime}(0)\right|=\frac{32\left(\lambda-\lambda_{1}\right)}{35 \lambda},
$$

since

$$
\left|F^{\prime}(\mu)\right|=-F^{\prime}(\mu)=\int_{0}^{2 \lambda^{-1}} x e^{-\mu x} f(x) d x
$$

is a decreasing function of $\mu$, and

$$
\left|F^{\prime}(0)\right|=\int_{0}^{2 \lambda^{-1}} x \frac{\lambda}{30}(2-\lambda x)^{3}\left[4+6 \lambda x+\lambda^{2} x^{2}\right] d x=\frac{16}{35 \lambda} .
$$

On the other hand

$$
\begin{aligned}
F(-\lambda)-F\left(-\lambda_{1}\right) & \\
& \geq\left(\lambda-\lambda_{1}\right)\left|F^{\prime}\left(-\lambda_{1}\right)\right| \\
& =\left(\lambda-\lambda_{1}\right) \int_{0}^{2 \lambda^{-1}} x e^{\lambda_{1} x} f(x) d x \\
& \geq\left(\lambda-\lambda_{1}\right) e^{\left(\lambda_{1}-\lambda\right) 2 \lambda^{-1}} \int_{0}^{2 \lambda^{-1}} x e^{\lambda x} f(x) d x \\
& =\left(\lambda-\lambda_{1}\right) e^{2 \lambda_{1} \lambda^{-1}-2} \int_{0}^{2 \lambda^{-1}} x e^{\lambda x} \frac{\lambda}{30}(2-\lambda x)^{3}\left[4+6 \lambda x+\lambda^{2} x^{2}\right] d x \\
& =\frac{16\left(\lambda-\lambda_{1}\right)}{15 \lambda} e^{2 \lambda_{1} \lambda^{-1}-2} .
\end{aligned}
$$

Thus (10.7) implies

$$
\exp \left(2 \lambda_{1} \lambda^{-1}-2\right) \leq \frac{6}{7}
$$

so that $\lambda_{1} \leq 0.792$. Finally, the falsity of (10.7) for $\lambda_{1} \leq 0.792$ may be verified by evaluating the function $F$ explicitly, via numerical integration. For example,

$$
F\left(-\frac{6}{7}\right)-F(-0.792) \geq 0.078
$$

while

$$
2\left(F(0)-F\left(\frac{6}{7}-0.783\right)\right) \leq 0.077
$$

so that $(10.7)$ is false for $0.792 \geq \lambda_{1} \geq 0.783$.

We deduce as follows. 
Lemma 10.1 Suppose that neither $\chi_{3}$ nor $\overline{\chi_{3}}$ is equal to $\chi_{1} \chi_{2}$ or $\chi_{1} \overline{\chi_{2}}$. Then $\lambda_{3} \geq \frac{6}{7}-\varepsilon$ for sufficiently large $q$.

We turn now to the case in which $\chi_{1} \chi_{2} \chi_{3}=\chi_{0}$, but the characters $\chi_{j}$ are all complex. Here we have

$$
\begin{aligned}
K\left(\beta_{1}+i \gamma_{2}+i \gamma_{3}, \chi_{2} \chi_{3}\right) & =K\left(\beta_{1}-i \gamma_{1}+i\left(\gamma_{1}+\gamma_{2}+\gamma_{3}\right), \overline{\chi_{1}}\right) \\
& \leq-\mathcal{L} \Re\{F(i \mu)\}+f(0)\left(\frac{\phi}{2}+\varepsilon\right) \mathcal{L}
\end{aligned}
$$

where $\mu=\mu_{1}+\mu_{2}+\mu_{3}$ with $\mu_{j}$ as in (6.2). Hence (10.4) may be sharpened to

$$
\Sigma_{2} \leq-\mathcal{L} \Re\{F(i \mu)\}+f(0)(3 \phi+\varepsilon) \mathcal{L},
$$

on re-defining $\varepsilon$. Since none of the $\chi_{j}$ are real, only the first term of (10.3) involves $\chi_{0}$, and so (10.5) is replaced by

$$
\Sigma_{3} \leq \mathcal{L} \Re\left\{F\left(-\lambda_{1}+i \mu\right)\right\}+f(0)\left(\frac{3 \phi}{2}+\varepsilon\right) \mathcal{L}
$$

We therefore deduce that (10.6) holds as before, providing that

$$
\max _{\mu \in \mathbb{R}} \Re\left\{F\left(-\lambda_{1}+i \mu\right)-2 F(i \mu)\right\} \leq \frac{f(0)}{6},
$$

for $0 \leq \lambda_{1} \leq \lambda$

If $\chi_{1} \chi_{2} \chi_{3}=\chi_{0}$, and exactly one character $\chi_{j}$ is real, we obtain

$$
\Sigma_{3} \leq \mathcal{L}\left[\Re\left\{F\left(-\lambda_{1}+i \mu\right)\right\}+\Re\left\{F\left(-\lambda_{1}+i \mu^{(j)}\right)\right\}\right]+f(0)\left(\frac{2 \phi}{2}+\varepsilon\right) \mathcal{L},
$$

where $\mu^{(j)}=\mu-2 \mu_{j}$. If $j=3$, say, then

$$
\begin{aligned}
K\left(\beta_{1}+i \gamma_{2}-i \gamma_{3}, \chi_{2} \overline{\chi_{3}}\right) & =K\left(\beta_{1}-i \gamma_{1}+i\left(\gamma_{1}+\gamma_{2}-2 \gamma_{3}\right), \overline{\chi_{1}}\right) \\
& \leq-\mathcal{L} \Re\left\{F\left(i \mu^{(3)}\right)\right\}+f(0)\left(\frac{\phi}{2}+\varepsilon\right) \mathcal{L},
\end{aligned}
$$

and similarly for $j=2$. If, on the other hand $j=1$ and $\mu_{1} \neq 0$, then

$$
\begin{aligned}
K\left(\beta_{1}+i \gamma_{2}+i \gamma_{3}, \chi_{2} \chi_{3}\right) & =K\left(\beta_{1}-i \gamma_{1}+i\left(\gamma_{1}+\gamma_{2}+\gamma_{3}\right), \overline{\chi_{1}}\right) \\
& \leq-\mathcal{L}\left[\Re\{F(i \mu)\}+\Re\left\{F\left(i \mu^{(j)}\right)\right\}\right]+f(0)\left(\frac{\phi}{2}+\varepsilon\right) \mathcal{L} .
\end{aligned}
$$

In each case we find that

$$
\Sigma_{2} \leq-\mathcal{L}\left[\Re\{F(i \mu)\}+\Re\left\{F\left(i \mu^{(j)}\right)\right\}\right]+f(0)(3 \phi+\varepsilon) \mathcal{L},
$$


with a new $\varepsilon$, and hence (10.8) still suffices for (10.6). Indeed there is a little to spare, since $\phi\left(\chi_{j}\right)=\frac{1}{4}<\frac{1}{3}$. When $\chi_{1} \chi_{2} \chi_{3}=\chi_{0}$ and all the $\chi_{j}$ are real, we find in the same way that

$$
\Sigma_{3} \leq \mathcal{L}\left[\Re\left\{F\left(-\lambda_{1}+i \mu\right)\right\}+\sum_{j=1}^{3} \Re\left\{F\left(-\lambda_{1}+i \mu^{(j)}\right)\right\}\right]+f(0) \varepsilon \mathcal{L}
$$

and

$$
\Sigma_{2} \leq-\mathcal{L}\left[\Re\{F(i \mu)\}+\sum_{j=1}^{3} \Re\left\{F\left(i \mu^{(j)}\right)\right\}\right]+f(0)(3 \phi+\varepsilon) \mathcal{L},
$$

providing that $\mu_{1} \neq 0$. In this case too, therefore (10.8) suffices for (10.6).

We therefore have the task of verifying the inequality (10.8). We begin by disposing of large $\mu$. We write

$$
F(s)=\int_{0}^{2 \lambda^{-1}} e^{-s x} f(x) d x
$$

where

$$
f(x)=\frac{\lambda}{30}\left(32-40 \lambda^{2} x^{2}+20 \lambda^{3} x^{3}-\lambda^{5} x^{5}\right),
$$

and integrate by parts to give

$$
\begin{aligned}
F(s)=\frac{16 \lambda}{15} s^{-1}-\frac{8 \lambda^{3}}{3} s^{-3} & +\left(4 \lambda^{4}+4 \lambda^{4} e^{-2 s / \lambda}\right) s^{-4} \\
& -4 \lambda^{6} s^{-4} \int_{0}^{2 \lambda^{-1}} x e^{-s x} d x .
\end{aligned}
$$

When $s=-\lambda_{1}+i \mu$ we have $\Re\left(s^{-1}\right) \leq 0$, and $\Re\left(s^{-3}\right) \geq 0$, for $\mu \geq \lambda_{1}$, so that

$$
\begin{aligned}
\Re(F(s)) & \leq 4 \lambda^{4}\left(e^{2}+1\right)|s|^{-4}+4 \lambda^{6}|s|^{-4} \int_{0}^{2 \lambda^{-1}} x e^{\lambda x} d x \\
& =8 \lambda^{4}\left(e^{2}+1\right)|s|^{-4} .
\end{aligned}
$$

On the other hand $\Re(F(i \mu)) \geq 0$ and $\frac{1}{6} f(0)=\frac{8 \lambda}{45}$. It therefore follows that (10.8) holds if $\mu \geq \lambda_{1}$ and

$$
8 \lambda^{4}\left(e^{2}+1\right) \mu^{-4} \leq \frac{8 \lambda}{45}
$$

whence $\mu \geq 4$ suffices.

For the remaining range of $\mu$ we observe that

$$
\left|F^{\prime}(s)\right|=\left|\int_{0}^{2 \lambda^{-1}} x e^{-s x} f(x) d x\right|
$$




$$
\begin{aligned}
& \leq \int_{0}^{2 \lambda^{-1}} x e^{\lambda x} f(x) d x \\
& =\frac{16}{15 \lambda}
\end{aligned}
$$

for $-\lambda \leq \Re(s) \leq 0$, and

$$
\left|F^{\prime}(i \mu)\right| \leq \int_{0}^{2 \lambda^{-1}} x f(x) d x=\frac{16}{35 \lambda},
$$

as a calculation based on Lemma 7.2 shows. Consequently, if we evaluate

$$
\mathcal{M}=\max \left\{\Re\left[F\left(-\lambda_{1}+i \mu\right)-2 F(i \mu)\right]:\left(\lambda_{1}, \mu\right) \in G\right\},
$$

where $G$ is the grid

$$
G=\left\{\left(\frac{m \lambda}{N}, \frac{n \lambda}{N}\right): 0 \leq m \leq N, 0 \leq n \leq 1+\frac{4 N}{\lambda}\right\}
$$

we will have

$$
\begin{aligned}
\max _{0 \leq \lambda_{1} \leq \lambda, 0 \leq \mu \leq 4} \Re\left\{F\left(-\lambda_{1}+i \mu\right)-2 F(i \mu)\right\} & \leq \mathcal{M}+\frac{16}{15} \cdot \frac{\sqrt{2}}{2 N}+2 \frac{16}{35} \cdot \frac{1}{2 N} \\
& \leq \mathcal{M}+\frac{5}{4 N} .
\end{aligned}
$$

We take $N=10$ and compute $\mathcal{M}$ by performing numerical integrations at each of the grid points. The result is that $\mathcal{M} \leq 0$, and since

$$
\frac{5}{40}<\frac{16 \lambda}{90}=\frac{1}{6} f(0)
$$

we see that the inequality (10.8) does indeed hold. We therefore deduce as follows.

Lemma 10.2 Suppose that $\chi_{1} \chi_{2} \chi_{3}=\chi_{0}$ and that either $\chi_{1}$ is complex or $\rho_{1}$ is complex. Then $\lambda_{3} \geq \frac{6}{7}-\varepsilon$ providing that $q$ is sufficiently large.

We turn now to the case in which $\chi_{1} \chi_{2} \chi_{3}=\chi_{0}$, and $\chi_{1}$ and $\rho_{1}$ are real. We use an argument analogous to the above, but incorporating the fact that $\phi=\frac{1}{4}$ for real characters. We now find that the condition

$$
\Re\left\{F\left(-\lambda_{1}+i \mu\right)-F(i \mu)\right\} \leq \frac{7}{24} f(0) \quad\left(\mu=\mu_{2}+\mu_{3}\right)
$$

suffices for (10.6), providing that $\chi_{2}$ and $\chi_{3}$ are complex. This time the estimate (10.9) shows that (10.12) holds for $\mu \geq 3.5$. Using

$$
\mathcal{M}=\max \left\{\Re\left[F\left(-\lambda_{1}+i \mu\right)-F(i \mu)\right]:\left(\lambda_{1}, \mu\right) \in G\right\},
$$


where

$$
G=\left\{\left(\frac{m \lambda}{N}, \frac{n \lambda}{N}\right): 0 \leq m \leq N, \frac{\alpha N}{\lambda} \leq n \leq 1+\frac{3.5 N}{\lambda}\right\}
$$

we find that

$$
\begin{aligned}
\Re\left\{F\left(-\lambda_{1}+i \mu\right)-F(i \mu)\right\} & \leq \mathcal{M}+\frac{16}{15} \cdot \frac{\sqrt{2}}{2 N}+\frac{16}{35} \cdot \frac{1}{2 N} \\
& \leq \mathcal{M}+\frac{1}{N}
\end{aligned}
$$

by (10.10) and (10.11). Taking $\alpha=1.25$ and $N=20$ we then calculate that $\mathcal{M} \leq 0.199$, so that (10.12) holds for $\mu \geq 1.25$.

To handle smaller values of $\mu$, for which (10.12) may be false, we return to (10.3), and replace $\beta_{1}$ by $\beta_{2}$. Since $\lambda^{\prime} \geq 1.294>\frac{6}{7}$, by Lemma 8.4 , only the zero $\rho_{1}$ need be considered for $\chi_{1}$. We now find that

$$
F\left(-\lambda_{2}\right)-F\left(\lambda_{1}-\lambda_{2}\right)-F(0)-F\left(\lambda_{3}-\lambda_{2}\right)+T+\left(\frac{7}{6}+\varepsilon\right) f(0) \geq 0
$$

where

$$
T=\Re\left\{-F(i \mu)-\frac{1}{2} F\left(\lambda_{1}-\lambda_{2}+i \mu\right)+\frac{1}{2} F\left(-\lambda_{2}+i \mu\right)-\frac{7}{48} f(0)\right\},
$$

with $\mu=\mu_{2}+\mu_{3}$. However (10.8) shows that

$$
\Re\left\{-F(i \mu)+\frac{1}{2} F\left(-\lambda_{2}+i \mu\right)\right\} \leq \frac{1}{12} f(0),
$$

while, in the usual way, we find

$$
\Re\left\{F\left(\lambda_{1}-\lambda_{2}+i \mu\right)\right\} \geq \mathcal{M}-\frac{5}{6 N} \quad(0 \leq \mu \leq 1.25)
$$

for

$$
\mathcal{M}=\min \{\Re[F(-t+i \mu)]:(t, \mu) \in G\}
$$

where

$$
G=\left\{\left(\frac{m \lambda}{N}, \frac{n \lambda}{N}\right): 0 \leq m \leq N, 0 \leq n \leq 1+\frac{1.25 N}{\lambda}\right\} .
$$

Taking $N=20$ we obtain $\mathcal{M} \geq 0.57$ and hence

$$
\Re\left\{F\left(\lambda_{1}-\lambda_{2}+i \mu\right)\right\} \geq 0.52 \geq-\frac{1}{8} f(0) \quad(0 \leq \mu \leq 1.25) .
$$

Thus $T \leq 0$, so that

$$
F\left(-\lambda_{2}\right)-F\left(\lambda_{1}-\lambda_{2}\right)-F(0)-F\left(\lambda_{3}-\lambda_{2}\right)+\lambda^{-1} f(0) \geq 0 .
$$


Since $F\left(\lambda_{1}-\lambda_{2}\right) \geq F(0)$ and

$$
\begin{aligned}
F\left(-\lambda_{2}\right)-F\left(\lambda_{3}-\lambda_{2}\right) & =\int_{0}^{2 \lambda^{-1}} e^{\lambda_{2} x}\left(1-e^{-\lambda_{3} x}\right) f(x) d x \\
& \leq \int_{0}^{2 \lambda^{-1}} e^{\lambda_{3} x}\left(1-e^{-\lambda_{3} x}\right) f(x) d x \\
& =F\left(-\lambda_{3}\right)-F(0)
\end{aligned}
$$

we deduce that

$$
F\left(-\lambda_{3}\right)-3 F(0)+\lambda^{-1} f(0) \geq 0 .
$$

Now Lemma 7.5 yields $F\left(-\lambda_{3}\right) \geq F(-\lambda)$, so that $\lambda_{3} \geq \lambda$ in this case too.

There remains the case in which $\chi_{1} \chi_{2} \chi_{3}=\chi_{0}$ with real characters $\chi_{j}$, and $\rho_{1}$ real. Here we use the inequality (10.1) in conjunction with the Dirichlet series (8.4) corresponding to the polynomial $P_{3}(X)$. We take $s=1+a \mathcal{L}^{-1}$, and find that

$$
\begin{aligned}
& P_{3}\left(\frac{a+\lambda_{1}}{a}\right)-P_{3}(1)-P_{3}\left(\frac{a+\lambda_{1}}{a+\lambda_{2}}\right)-P_{3}\left(\frac{a+\lambda_{1}}{a+\lambda_{3}}\right) \\
& \quad+\frac{1}{2}\left\{P^{*}\left(\mu_{2}+\mu_{3}\right)+P^{*}\left(\mu_{2}-\mu_{3}\right)\right\}+\left(a+\lambda_{1}\right)\left(\frac{6 \phi}{2}+\varepsilon\right) \geq 0
\end{aligned}
$$

with $\phi=\frac{1}{4}$ and

$P^{*}(t)=\Re\left\{P_{3}\left(\frac{a+\lambda_{1}}{a+i t}\right)-P_{3}\left(\frac{a+\lambda_{1}}{a+\lambda_{1}+i t}\right)-P_{3}\left(\frac{a+\lambda_{1}}{a+\lambda_{2}+i t}\right)-P_{3}\left(\frac{a+\lambda_{1}}{a+\lambda_{3}+i t}\right)\right\}$.

here, for example, we have used the fact that

$$
\chi_{1}(n) \chi_{2}(n) n^{-i \gamma_{2}}=\chi_{3}(n) n^{-i \gamma_{2}}
$$

produces terms

$$
\begin{aligned}
& \Re\left\{P_{3}\left(\frac{a+\lambda_{1}}{\left(s+i \gamma_{2}-\rho_{3}\right) \mathcal{L}}\right)+P_{3}\left(\frac{a+\lambda_{1}}{\left(s+i \gamma_{2}-\overline{\rho_{3}}\right) \mathcal{L}}\right)\right\} \\
& \leq \frac{1}{2} \Re\left\{P_{3}\left(\frac{a+\lambda_{1}}{\left(s+i \gamma_{2}-\rho_{3}\right) \mathcal{L}}\right)+P_{3}\left(\frac{a+\lambda_{1}}{\left(s+i \gamma_{2}-\overline{\rho_{3}}\right) \mathcal{L}}\right)\right\}
\end{aligned}
$$

if $\rho_{3} \neq \overline{\rho_{3}}$, and

$$
\Re\left\{P_{3}\left(\frac{a+\lambda_{1}}{\left(s+i \gamma_{2}-\rho_{3}\right) \mathcal{L}}\right)\right\}=\frac{1}{2} \Re\left\{P_{3}\left(\frac{a+\lambda_{1}}{\left(s+i \gamma_{2}-\rho_{3}\right) \mathcal{L}}\right)+P_{3}\left(\frac{a+\lambda_{1}}{\left(s+i \gamma_{2}-\overline{\rho_{3}}\right) \mathcal{L}}\right)\right\}
$$

if $\rho_{3}$ is real. This yields a contribution

$$
-\frac{1}{2} \Re\left\{P_{3}\left(\frac{a+\lambda_{1}}{a+\lambda_{3}+i\left(\mu_{2}-\mu_{3}\right)}\right)\right\}-\frac{1}{2} \Re\left\{P_{3}\left(\frac{a+\lambda_{1}}{a+\lambda_{3}+i\left(\mu_{2}+\mu_{3}\right)}\right)\right\}
$$


to $(10.13)$. The analysis leading to Lemma 8.3 shows that $P^{*}(t) \leq 0$ providing that

$$
a^{-3} \leq\left(a+\lambda_{1}\right)^{-3}+\left(a+\lambda_{2}\right)^{-3}+\left(a+\lambda_{3}\right)^{-3} .
$$

In this case we will have

$$
P_{3}\left(\frac{a+\lambda_{1}}{a}\right)-P_{3}(1)-P_{3}\left(\frac{a+\lambda_{1}}{a+\lambda_{2}}\right)-P_{3}\left(\frac{a+\lambda_{1}}{a+\lambda_{3}}\right)+\left(a+\lambda_{1}\right)\left(\frac{3}{4}+\varepsilon\right) \geq 0 .
$$

Assuming that $\lambda_{3} \leq \lambda=\frac{6}{7}$, we take $a=3 \lambda$, so that

$$
\left(a+\lambda_{1}\right)^{-3}+\left(a+\lambda_{2}\right)^{-3}+\left(a+\lambda_{3}\right)^{-3} \geq 3(4 \lambda)^{-3} \geq(3 \lambda)^{-3} .
$$

Thus (10.14) holds, whence

$$
P_{3}\left(\frac{a+\lambda_{1}}{a}\right)-P_{3}(1)-2 P_{3}\left(\frac{a+\lambda_{1}}{a+\lambda}\right)+\left(a+\lambda_{1}\right)\left(\frac{3}{4}+\varepsilon\right) \geq 0 .
$$

We write, temporarily, $\lambda_{1}=\delta \lambda$, so that $0 \leq \delta \leq 1$, and

$$
P_{3}\left(\frac{3+\delta}{3}\right)-\frac{8}{3}-2 P_{3}\left(\frac{3+\delta}{4}\right)+(3+\delta)\left(\frac{9}{14}+\varepsilon\right) \geq 0
$$

This may be re-written as

$$
A(3+\delta)+B(3+\delta)^{2}+C(3+\delta)^{3} \geq \frac{8}{3}
$$

with

$$
\begin{array}{r}
A=\frac{1}{3}-2 \cdot \frac{1}{4}+\frac{9}{14}+\varepsilon>0 \\
B=\frac{1}{9}-2 \cdot \frac{1}{16}<0 \\
C=\frac{2}{3}\left(\frac{1}{27}-2 \cdot \frac{1}{64}\right)>0 .
\end{array}
$$

Since $0 \leq \delta \leq 1$, we have

$$
\begin{aligned}
A(3+\delta)+B(3+\delta)^{2}+C(3+\delta)^{3} & \leq 4 A+9 B+64 C \\
& =\frac{9193}{4536}+4 \varepsilon \\
& <\frac{8}{3} .
\end{aligned}
$$

This contradiction shows finally that $\lambda_{3} \geq \frac{6}{7}$ in the present case too.

We have now covered all possible cases, and we conclude as follows.

Lemma 10.3 Let $\varepsilon>0$. Then if $q$ is sufficiently large we have $\lambda_{3} \geq \frac{6}{7}-\varepsilon$. 


\section{The Old Zero Density Estimate}

The zero density estimate used by Graham $[11 ;(24)]$ takes the form

$$
N(\lambda) \leq(1+\varepsilon) \frac{167}{18} e^{7 \lambda / 2} \quad(q \geq q(\varepsilon))
$$

where $N(\lambda)$ is the number of distinct non-principal characters modulo $q$ for which $L(s, \chi)$ has a zero in the region $\sigma \geq 1-\mathcal{L}^{-1} \lambda,|t| \leq 1$. We number the characters $\chi^{(1)}, \chi^{(2)}, \ldots, \chi^{(N)}$, where $N=N(\lambda)$, and for each $\chi^{(k)}$ we choose a corresponding zero $\rho^{(k)}$, and write

$$
\rho^{(k)}=\beta^{(k)}+i \gamma^{(k)}, \quad \beta^{(k)}=1-\mathcal{L}^{-1} \lambda^{(k)}, \quad \gamma^{(k)}=\mathcal{L}^{-1} \mu^{(k)},
$$

in analogy to (6.2).

At the time of Graham's work only a weaker form of Burgess's bound was available, corresponding to $\phi=\frac{3}{8}$ in Lemma 2.5. However it is a trivial matter to use $\phi=\frac{1}{3}$ instead, and hence to reduce the exponent $\frac{7}{2}$ in (11.1) to $\frac{28}{9}$. We shall make a second minor change in Graham's argument, producing a bound corresponding to

$$
N(\lambda) \leq(1+\varepsilon) \frac{225}{44 \lambda}\left(e^{7 \lambda / 2}-e^{163 \lambda / 100}\right) \quad(q \geq q(\varepsilon)) .
$$

This is of comparable strength to (11.1).

We shall also describe a more significant modification to Graham's approach, which shows, in effect that

$$
\sum_{k \leq N} \lambda^{(k)} /\left\{\exp \left(\frac{7 \lambda^{(k)}}{2}\right)-\exp \left(\frac{163 \lambda^{(k)}}{100}\right)\right\} \leq(1+\varepsilon) \frac{225}{44} \quad(q \geq q(\varepsilon)) .
$$

Since the function $\lambda /\left\{e^{A \lambda}-e^{B \lambda}\right\}$ is decreasing with $\lambda$ when $A>B>0$, our new bound obviously includes (11.2). Moreover we can take $N=N(\lambda)$ for any $\lambda \leq \frac{1}{3} \log \log \mathcal{L}$, and still have the same bound on the right.

In fact there are two parameters at our disposal. Our result is as follows.

Lemma 11.1 Let constants $\varepsilon, c_{1}, c_{2}>0$ be given, and define

$$
\phi=\max _{\chi(\bmod q)} \phi(\chi) .
$$

Then if $\lambda_{0}=\frac{1}{3} \log \log \mathcal{L}$, we have

$$
\begin{gathered}
\sum_{k \leq N\left(\lambda_{0}\right)} \lambda^{(k)} /\left\{\exp \left(\left(4 \phi+6 c_{1}+2 c_{2}\right) \lambda^{(k)}\right)-\exp \left(\left(2 \phi+4 c_{1}\right) \lambda^{(k)}\right)\right\} \\
\leq \frac{2 \phi+2 c_{1}+c_{2}}{4 c_{1} c_{2}}+\varepsilon
\end{gathered}
$$


for $q \geq q\left(\varepsilon, c_{1}, c_{2}\right)$. In particular, taking $\phi \leq \frac{1}{3}$, and $c_{1}=\frac{1}{10}, c_{2}=\frac{1}{4}$, we have

$$
\sum_{k \leq N\left(\lambda_{0}\right)} \lambda^{(k)} /\left\{\exp \left(\frac{73 \lambda^{(k)}}{30}\right)-\exp \left(\frac{16 \lambda^{(k)}}{15}\right)\right\} \leq \frac{67}{6}+\varepsilon,
$$

and hence

$$
N(\lambda) \leq(1+\varepsilon) \frac{67}{6 \lambda}\left(e^{73 \lambda / 30}-e^{16 \lambda / 15}\right),
$$

for any $\lambda \leq \frac{1}{3} \log \log \mathcal{L}$, and $q \geq q(\varepsilon)$.

The above choices for $c_{1}$ and $c_{2}$ are roughly optimal for our applications. A table of bounds for $N(\lambda)$, derived from(11.5), is given as Table 12 .

For the proof we take parameters $U=q^{u}, V=q^{v}, W=q^{w}$, and $X=q^{x}$, with constant exponents $0<u<v$, and $w, x>0$, to be specified later, and we define

$$
\psi_{d}=\left\{\begin{array}{cc}
\mu(d), & 1 \leq d \leq U, \\
\mu(d) \frac{\log V / d}{\log V / U}, & U \leq d \leq V, \\
0, & d \geq V,
\end{array}\right.
$$

and

$$
\theta_{d}=\left\{\begin{array}{cc}
\mu(d) \frac{\log W / d}{\log W}, & 1 \leq d \leq W, \\
0, & d \geq W .
\end{array}\right.
$$

We take $\chi=\chi^{(k)}, \rho=\rho^{(k)}$ and consider

$$
\begin{aligned}
\sum_{n=1}^{\infty}\left(\sum_{d \mid n} \psi_{d}\right)\left(\sum_{d \mid n} \theta_{d}\right) \chi(n) n^{-\rho} e^{-n / X} & \\
= & \frac{1}{2 \pi i} \int_{1-i \infty}^{1+i \infty} L(s+\rho, \chi) \Gamma(s) X^{s} F(s+\rho) d s
\end{aligned}
$$

where

$$
F(s)=\sum_{v \leq V, w \leq W} \psi_{v} \theta_{w} \chi([v, w])[v, w]^{-s} .
$$

(Here $[v, w]$ denotes the least common multiple of $v$ and $w$.) We move the line of integration to $\Re s=1-\beta-\frac{1}{k}$, where $\beta=\Re \rho$, and $k$ is a positive integer constant which we shall specify in due course. According to Lemma 2.5 we have

$$
L(s+\rho, \chi) \ll_{k} q^{\phi / k+1 / k^{2}}(1+|t|) .
$$

Here we have chosen $\varepsilon=\left(2 k^{2}\right)^{-1}$. Moreover we have $\Gamma(s) \ll e^{-|t|}$ and

$$
F(s+\rho) \ll \sum_{v, w}[v, w]^{-1+1 / k} \ll \sum_{n \leq V W} n^{-1+1 / k} d(n)^{2} \ll(V W)^{1 / k} \mathcal{L}^{2} .
$$




\begin{tabular}{cr}
$\lambda$ & $N(\lambda)$ \\
\hline 0.800 & 65 \\
0.825 & 68 \\
0.850 & 71 \\
0.875 & 74 \\
0.900 & 78 \\
0.925 & 82 \\
0.950 & 86 \\
0.975 & 90 \\
1.000 & 94 \\
1.025 & 99 \\
1.050 & 104 \\
1.075 & 109 \\
1.100 & 114 \\
1.125 & 120 \\
1.150 & 126 \\
1.175 & 132 \\
1.200 & 139 \\
1.225 & 145 \\
1.250 & 153 \\
1.275 & 160 \\
1.300 & 168 \\
1.325 & 177 \\
1.350 & 186 \\
1.375 & 195 \\
1.400 & 205
\end{tabular}

\begin{tabular}{cc}
$\lambda$ & $N(\lambda)$ \\
\hline 1.425 & 215 \\
1.450 & 226 \\
1.475 & 237 \\
1.500 & 249 \\
1.525 & 262 \\
1.550 & 275 \\
1.575 & 289 \\
1.600 & 304 \\
1.625 & 319 \\
1.650 & 335 \\
1.675 & 352 \\
1.700 & 370 \\
1.725 & 389 \\
1.750 & 409 \\
1.775 & 430 \\
1.800 & 452 \\
1.825 & 476 \\
1.850 & 500 \\
1.875 & 526 \\
1.900 & 553 \\
1.925 & 582 \\
1.950 & 612 \\
1.975 & 644 \\
2.000 & 678
\end{tabular}

Table 12: The Old Zero Density Bound 
It follows that

$$
\begin{aligned}
\frac{1}{2 \pi i} \int_{1-\beta-1 / k-i \infty}^{1-\beta-1 / k+i \infty} L(s+\rho, \chi) & \Gamma(s) X^{s} F(s+\rho) d s \\
& \ll\left(q^{\phi} V W X^{-1}\right)^{1 / k} q^{1 / k^{2}} \mathcal{L}^{2} X^{1-\beta} \\
& \ll\left(q^{\phi} V W X^{-1}\right)^{1 / k} q^{2 / k^{2}}
\end{aligned}
$$

since $X=q^{x}$ and $1-\beta \ll \mathcal{L}^{-1} \log \log \mathcal{L}$. On choosing

$$
\frac{2}{k}<x-v-w-\phi
$$

we deduce that

$$
\sum_{n=1}^{\infty}\left(\sum_{d \mid n} \psi_{d}\right)\left(\sum_{d \mid n} \theta_{d}\right) \chi(n) n^{-\rho} e^{-n / X}=O\left(\mathcal{L}^{-1}\right)
$$

providing that

$$
x>v+w+\phi
$$

According to (11.6) we have

$$
\sum_{d \mid n} \psi_{d}=0 \quad(2 \leq n \leq U)
$$

whence

$$
\begin{aligned}
& \sum_{n=1}^{\infty}\left(\sum_{d \mid n} \psi_{d}\right)\left(\sum_{d \mid n} \theta_{d}\right) \chi(n) n^{-\rho} e^{-n / X} \\
& =e^{-\mathcal{L}^{2} / U}+\sum_{n=1}^{\infty}\left(\sum_{d \mid n} \psi_{d}\right)\left(\sum_{d \mid n} \theta_{d}\right) \chi(n) n^{-\rho}\left(e^{-n / X}-e^{-n \mathcal{L}^{2} / U}\right) \\
& \quad+O\left(\sum_{n>U} d^{2}(n) e^{-n \mathcal{L}^{2} / U}\right) \\
& =1+\sum_{n=1}^{\infty}\left(\sum_{d \mid n} \psi_{d}\right)\left(\sum_{d \mid n} \theta_{d}\right) \chi(n) n^{-\rho}\left(e^{-n / X}-e^{-n \mathcal{L}^{2} / U}\right)+O\left(\mathcal{L}^{-1}\right)
\end{aligned}
$$

In view of (11.9) we conclude that

$$
1 \leq\left\{1+O\left(\mathcal{L}^{-1}\right)\right\}\left|\sum_{n=1}^{\infty}\left(\sum_{d \mid n} \psi_{d}\right)\left(\sum_{d \mid n} \theta_{d}\right) \chi(n) n^{-\rho}\left(e^{-n / X}-e^{-n \mathcal{L}^{2} / U}\right)\right|^{2} .
$$

We now multiply by non-negative weights $w_{\chi}$ for the various characters $\chi^{(k)}$, and sum to give

$$
\sum_{\chi} w_{\chi} \leq\left\{1+O\left(\mathcal{L}^{-1}\right)\right\} \sum_{\chi}\left|\sum_{n} a_{n \chi} b_{n}\right|^{2}
$$


where

$$
a_{n \chi}=w_{\chi}^{1 / 2}\left(\sum_{d \mid n} \theta_{d}\right) \chi(n) n^{\frac{1}{2}-\rho}\left(e^{-n / X}-e^{-n \mathcal{L}^{2} / U}\right)^{1 / 2},
$$

and

$$
b_{n}=\left(\sum_{d \mid n} \psi_{d}\right) n^{-\frac{1}{2}}\left(e^{-n / X}-e^{-n \mathcal{L}^{2} / U}\right)^{1 / 2} .
$$

At this point we use the duality principle: if

$$
\sum_{n}\left|\sum_{\chi} A_{n \chi} C_{\chi}\right|^{2} \leq \mathcal{B} \sum_{\chi}\left|C_{\chi}\right|^{2}
$$

for all choices of the coefficients $C_{\chi}$, then

$$
\sum_{\chi}\left|\sum_{n} A_{n \chi} B_{n}\right|^{2} \leq \mathcal{B} \sum_{n}\left|B_{n}\right|^{2}
$$

for all choices of the $B_{n}$ 's. In our case we will obtain

$$
\sum_{\chi} w_{\chi} \leq\left\{1+O\left(\mathcal{L}^{-1}\right)\right\} \mathcal{B} \sum_{n=1}^{\infty}\left(\sum_{d \mid n} \psi_{d}\right)^{2} n^{-1}\left(e^{-n / X}-e^{-n \mathcal{L}^{2} / U}\right)
$$

Here we use Graham's estimate [10; page 84], namely

$$
\sum_{n \leq N}\left(\sum_{d \mid n} \psi_{d}\right)^{2}=\left\{\begin{array}{cc}
1, & 1 \leq N \leq U \\
\frac{N \log (N / U)}{(\log V / U)^{2}}+O\left(\frac{N}{(\log V / U)^{2}}\right), & U \leq N \leq V \\
\frac{N}{\log V / U}+O\left(\frac{N}{(\log V / U)^{2}}\right), & N \geq V
\end{array}\right.
$$

Now an easy summation by parts yields

$$
\sum_{n=1}^{\infty}\left(\sum_{d \mid n} \psi_{d}\right)^{2} n^{-1}\left(e^{-n / X}-e^{-n \mathcal{L}^{2} / U}\right)=\left(1+O\left(\mathcal{L}^{-1}\right)\right) \frac{2 x-u-v}{2(v-u)}
$$

since, according to (11.10), we have $x>v$.

It remains therefore to find a bound $\mathcal{B}$ such that

$$
\sum_{n}\left|\sum_{\chi} a_{n \chi} C_{\chi}\right|^{2} \leq \mathcal{B} \sum_{\chi}\left|C_{\chi}\right|^{2}
$$

If we expand the right hand side, the non-diagonal terms are of the form

$$
C_{\chi} \overline{C_{\chi^{\prime}}}\left(w_{\chi} w_{\chi^{\prime}}\right)^{1 / 2} \sum_{n=1}^{\infty}\left(\sum_{d \mid n} \theta_{d}\right)^{2} \chi(n) \overline{\chi^{\prime}}(n) n^{1-\rho-\overline{\rho^{\prime}}}\left(e^{-n / X}-e^{-n \mathcal{L}^{2} / U}\right) .
$$


In analogy to our treatment of (11.8) we will have

$$
\begin{array}{r}
\sum_{n=1}^{\infty}\left(\sum_{d \mid n} \theta_{d}\right)^{2} \chi(n) \overline{\chi^{\prime}}(n) n^{1-\rho-\overline{\rho^{\prime}}}\left(e^{-n / X}-e^{-n \mathcal{L}^{2} / U}\right) \\
\ll\left(q^{\phi} W^{2} U^{-1}\right)^{1 / k} q^{2 / k^{2}}
\end{array}
$$

for a suitable positive integer constant $k$. This will be $O\left(\mathcal{L}^{-1}\right)$, providing that

$$
u>2 w+\phi
$$

and $k$ is chosen large enough. The non-diagonal terms therefore contribute

$$
\ll \mathcal{L}^{-1}\left(\sum_{\chi}\left|C_{\chi}\right| w_{\chi}^{1 / 2}\right)^{2} \ll \mathcal{L}^{-1}\left(\sum_{\chi}\left|C_{\chi}\right|^{2}\right)\left(\sum_{\chi} w_{\chi}\right)
$$

to $(11.15)$

The typical diagonal term on the left of (11.15) is

$$
\left|C_{\chi}\right|^{2} w_{\chi} \sum_{n=1}^{\infty}\left(\sum_{d \mid n} \theta_{d}\right)^{2} \chi_{0}(n) n^{1-2 \beta}\left(e^{-n / X}-e^{-n \mathcal{L}^{2} / U}\right) .
$$

We now observe that $\theta_{d}$ is the special case of $\psi_{d}$ in which $U=1$ and $V=W$. Hence Graham's estimate (11.13) yields

$$
\sum_{n \leq N}\left(\sum_{d \mid n} \theta_{d}\right)^{2}=\left\{\begin{array}{cc}
\frac{N \log N}{(\log W)^{2}}+O\left(\frac{N}{(\log W)^{2}}\right), & 1 \leq N \leq W \\
\frac{N}{\log W}+O\left(\frac{N}{(\log W)^{2}}\right), & N \geq W
\end{array}\right.
$$

so that

$$
\begin{aligned}
\sum_{n=1}^{\infty}\left(\sum_{d \mid n} \theta_{d}\right)^{2} n^{1-2 \beta}\left(e^{-n / X}-e^{-n \mathcal{L}^{2} / U}\right) & \\
= & \left\{1+O\left(\mathcal{L}^{-1}\right)\right\} \frac{\Gamma(2-2 \beta)}{\log W}\left(X^{2-2 \beta}-\left(\frac{U}{\mathcal{L}^{2}}\right)^{2-2 \beta}\right)
\end{aligned}
$$

Here we have used the condition $u>w$ (see (11.16)). Since

$$
U^{2-2 \beta}(1-\beta) \mathcal{L} \ll X^{2-2 \beta}-U^{2-2 \beta}
$$

we find that

$$
\begin{aligned}
X^{2-2 \beta}-\left(\frac{U}{\mathcal{L}^{2}}\right)^{2-2 \beta} & =X^{2-2 \beta}-U^{2-2 \beta}(1+O((1-\beta) \log \mathcal{L})) \\
& =\left\{1+O\left(\mathcal{L}^{-1} \log \mathcal{L}\right)\right\}\left(X^{2-2 \beta}-U^{2-2 \beta}\right) .
\end{aligned}
$$


Moreover we have

$$
\Gamma(2-2 \beta)=\left\{1+O\left(\mathcal{L}^{-1} \log \log \mathcal{L}\right)\right\} \frac{1}{2-2 \beta},
$$

since $\beta=1+O\left(\mathcal{L}^{-1} \log \log \mathcal{L}\right)$. It follows that $(11.17)$ is

$$
\left\{1+O\left(\mathcal{L}^{-1} \log \mathcal{L}\right)\right\} \frac{X^{2-2 \beta}-U^{2-2 \beta}}{(2-2 \beta) \mathcal{L} w} .
$$

We therefore take

$$
w_{\chi}=\frac{(2-2 \beta) \mathcal{L} w}{X^{2-2 \beta}-U^{2-2 \beta}}
$$

and conclude that

$$
\mathcal{B}=1+O\left(\mathcal{L}^{-1} \sum w_{\chi}\right)+O\left(\mathcal{L}^{-1} \log \mathcal{L}\right)
$$

is an admissable choice.

We now deduce that

$$
\begin{aligned}
\sum w_{\chi} & \leq\left\{1+O\left(\mathcal{L}^{-1} \log \mathcal{L}\right)\right\}\left\{1+O\left(\mathcal{L}^{-1} \sum w_{\chi}\right)\right\} \frac{2 x-u-v}{2(v-u)} \\
& =\left\{1+O\left(\mathcal{L}^{-1} \log \mathcal{L}\right)\right\} \frac{2 x-u-v}{2(v-u)}+O\left(\mathcal{L}^{-1} \sum w_{\chi}\right)
\end{aligned}
$$

and hence

$$
\sum w_{\chi} \leq\left\{1+O\left(\mathcal{L}^{-1} \log \mathcal{L}\right)\right\} \frac{2 x-u-v}{2(v-u)} .
$$

It remains therefore to choose the constants $u, v, w, x$, subject to the conditions $0<u<v ; w, x>0 ; x>v+w+\phi$ and $u>2 w+\phi$. We wish to maximize

$$
\frac{w(v-u)}{2 x-u-v}\left\{X^{2-2 \beta}-U^{2-2 \beta}\right\}^{-1}
$$

subject to these constraints. We write $u=\phi+2 c_{1}, v=u+c_{2}$ with $c_{1}, c_{2}>0$. The expression (11.19) is decreasing with respect to $x$, and we therefore choose $x=v+w+\phi+\varepsilon$. Then (11.19) becomes

$$
\frac{w c_{2}}{c_{2}+2 w+2 \phi+2 \varepsilon} U^{2 \beta-2}\left\{\exp \left[(2-2 \beta)\left(c_{2}+w+\phi+\varepsilon\right)\right]-1\right\}^{-1},
$$

which is decreasing with respect to $c_{1}$. We therefore take $c_{1}=w+\varepsilon$. Feeding the values $v=\phi+2 c_{1}+c_{2}, u=\phi+2 c_{1}, w=c_{1}-\varepsilon, x=2 \phi+3 c_{1}+c_{2}$ into (11.18) then produces (11.3), with a new value for $\varepsilon$.

We remark in conclusion that the choice $c_{1}=c_{2}=\varepsilon$ in Lemma 11.1 yields a bound

$$
N(\lambda) \ll q^{(4 \phi+8 \varepsilon) \lambda} \quad\left(\lambda \leq \frac{1}{3} \log \log \mathcal{L}\right)
$$


which is distinctly sharper than the estimate $O\left(q^{(2+\varepsilon) \lambda}\right)$ which follows from Jutila's zero-density theorem [21; Theorem 1]

$$
\sum_{\chi(\bmod q)} N(\sigma, T, \chi) \ll(q T)^{(2+\varepsilon)(1-\sigma)} \quad\left(T \geq 1, \frac{4}{5} \leq \sigma \leq 1\right) .
$$

However (11.20) holds for a very restricted range of $\lambda$.

We also remark that the condition $\lambda \leq \lambda_{0}$ in (11.5) may be dropped, in view of the bound (1.4). This implies $N(\lambda) \ll q^{(72 / 30+\varepsilon) \lambda}$, so that (11.5) holds automatically for $\lambda \geq \lambda_{0}$. Similarly, in (11.4), the contribution from zeros with $\lambda^{(k)} \geq \lambda_{0}$ is $o(1)$. We state this formally.

Lemma 11.2 The estimate (11.4) holds when summed over all non-principal characters modulo $q$. 


\section{A New Zero Density Estimate}

In this section we give a new type of zero density estimate, which is non-trivial only for $\sigma=1+O\left(\mathcal{L}^{-1}\right)$, but is sharper than Lemma 11.1 when $\sigma$ is close to 1 . The argument is more closely related to that of $\S \S 6-10$ than to that of $\S 11$.

We adopt the notation of $\S 11$, but we restrict $\lambda$ to the range $0 \leq \lambda \leq 2$. It follows from the conventions of $\S 6$ that all zeros $\rho$, of any L-function to modulus $q$, satisfy either $|\gamma| \geq 10$ or $\beta \leq 1-\mathcal{L}^{-1} \lambda_{1}$. We therefore have

$$
\begin{aligned}
K\left(\beta_{1}+i \gamma^{(j)}, \chi^{(j)}\right) & \leq-\mathcal{L} F\left(\lambda^{(j)}-\lambda_{1}\right)+f(0)\left(\frac{\phi}{2}+\varepsilon\right) \mathcal{L} \\
& \leq-\mathcal{L} F\left(\lambda-\lambda_{1}\right)+f(0)\left(\frac{\phi}{2}+\varepsilon\right) \mathcal{L}
\end{aligned}
$$

whence

$$
\begin{aligned}
\mathcal{L} N\left\{F\left(\lambda-\lambda_{1}\right)-f(0)\right. & \left.\left(\frac{\phi}{2}+\varepsilon\right)\right\} \\
\leq & -\sum_{j \leq N} K\left(\beta_{1}+i \gamma^{(j)}, \chi^{(j)}\right) \\
& =-\sum_{n=1}^{\infty} \Lambda(n) n^{-\beta_{1}} f\left(\mathcal{L}^{-1} \log n\right) \Re\left\{\sum_{j \leq N} \chi^{(j)} n^{-i \gamma^{(j)}}\right\} \\
& \leq \sum_{n=1}^{\infty} \Lambda(n) n^{-\beta_{1}} f\left(\mathcal{L}^{-1} \log n\right)\left|\sum_{j \leq N} \chi^{(j)} n^{-i \gamma^{(j)}}\right|
\end{aligned}
$$

We shall suppose that

$$
F\left(\lambda-\lambda_{1}\right) \geq f(0)\left(\frac{\phi}{2}+\varepsilon\right) .
$$

Then, on applying Cauchy's inequality, we find that

$$
\mathcal{L}^{2} N^{2}\left\{F\left(\lambda-\lambda_{1}\right)-f(0)\left(\frac{\phi}{2}+\varepsilon\right)\right\}^{2} \leq \Sigma_{1} \Sigma_{2},
$$

with

$$
\Sigma_{1}=\sum_{n=1}^{\infty} \Lambda(n) n^{-\beta_{1}} f\left(\mathcal{L}^{-1} \log n\right)=K\left(\beta_{1}, \chi_{0}\right)
$$

and

$$
\begin{aligned}
\Sigma_{2} & =\sum_{n=1}^{\infty} \Lambda(n) n^{-\beta_{1}} f\left(\mathcal{L}^{-1} \log n\right)\left|\sum_{j \leq N} \chi^{(j)} n^{-i \gamma^{(j)}}\right|^{2} \\
& =\sum_{j, k \leq N} K\left(\beta_{1}+i \gamma^{(j)}-i \gamma^{(k)}, \chi^{(j)} \bar{\chi}^{(k)}\right) .
\end{aligned}
$$


The terms in $\Sigma_{2}$ with $j=k$ are just

$$
K\left(\beta_{1}, \chi_{0}\right) \leq \mathcal{L} F\left(-\lambda_{1}\right)+f(0) \varepsilon \mathcal{L}
$$

by Lemma 5.3 , while the remaining terms have

$$
K\left(\beta_{1}+i \gamma^{(j)}-i \gamma^{(k)}, \chi^{(j)} \bar{\chi}^{(k)}\right) \leq f(0)\left(\frac{\phi}{2}+\varepsilon\right) \mathcal{L}
$$

On choosing a new value for $\varepsilon$, we conclude that

$$
N^{2}\left\{F\left(\lambda-\lambda_{1}\right)-\frac{\phi}{2} f(0)\right\}^{2} \leq F\left(-\lambda_{1}\right)\left\{N F\left(-\lambda_{1}\right)+\left(N^{2}-N\right) \frac{\phi}{2} f(0)\right\}+\varepsilon,
$$

and hence that

$$
\begin{aligned}
N\left\{\left[F\left(\lambda-\lambda_{1}\right)-\frac{\phi}{2} f(0)\right]^{2}-F\right. & \left.\left(-\lambda_{1}\right) \frac{\phi}{2} f(0)\right\} \\
& \leq F\left(-\lambda_{1}\right)\left[F\left(-\lambda_{1}\right)-\frac{\phi}{2} f(0)\right]+\varepsilon
\end{aligned}
$$

for sufficiently large $q$. We therefore have the following.

Lemma 12.1 Let $\varepsilon>0$ and $0 \leq \lambda \leq 2$. Suppose that

$$
F\left(\lambda-\lambda_{1}\right)>f(0) / 6
$$

and

$$
\left(F\left(\lambda-\lambda_{1}\right)-f(0) / 6\right)^{2}>F\left(-\lambda_{1}\right) f(0) / 6
$$

Then

$$
N(\lambda) \leq \frac{F\left(-\lambda_{1}\right)\left[F\left(-\lambda_{1}\right)-f(0) / 6\right]}{\left[F\left(\lambda-\lambda_{1}\right)-f(0) / 6\right]^{2}-F\left(-\lambda_{1}\right) f(0) / 6}+\varepsilon
$$

for $q \geq q(f, \varepsilon)$.

We shall apply this using the functions from Lemma 7.1, given in terms of $\theta$ and $\hat{\lambda}$, say. The optimal choices are very close to

$$
\theta=\frac{1}{2} \lambda-\frac{1}{6} \lambda_{1}+1.7, \quad \hat{\lambda}=\lambda .
$$

A table of bounds for $N(\lambda)$ obtained from these values is given as Table 13. One sees that the bound is worse than that produced by Lemma 11.1 (see Table 12) as soon as $\lambda \geq 1.1$ in general, and for $\lambda \geq 1.225$ when $\lambda_{1} \geq 0.348$. However for smaller values of $\lambda$ the new estimate is substantially better. Indeed the results compare reasonably well with the bound $N(\lambda) \leq 4$ for $\lambda \leq 0.857$ implicit in Lemma 10.3. 


\begin{tabular}{c|r|r|r|r|r|r} 
& $\lambda_{1}=0$ & $\lambda_{1}=0.348$ & $\lambda_{1}=0.4$ & $\lambda_{1}=0.44$ & $\lambda_{1}=0.5$ & $\lambda_{1}=0.58$ \\
\hline$\lambda$ & $N(\lambda)$ & $N(\lambda)$ & $N(\lambda)$ & $N(\lambda)$ & $N(\lambda)$ & $N(\lambda)$ \\
\hline 0.800 & 11 & 8 & 8 & 8 & 7 & 7 \\
0.825 & 12 & 9 & 9 & 8 & 8 & 8 \\
0.850 & 14 & 10 & 9 & 9 & 9 & 8 \\
0.875 & 16 & 11 & 10 & 10 & 9 & 9 \\
0.900 & 18 & 12 & 11 & 11 & 10 & 10 \\
0.925 & 21 & 13 & 12 & 12 & 11 & 11 \\
0.950 & 25 & 15 & 14 & 13 & 12 & 12 \\
0.975 & 30 & 16 & 15 & 15 & 14 & 13 \\
1.000 & 38 & 18 & 17 & 16 & 15 & 14 \\
1.025 & 49 & 21 & 19 & 18 & 17 & 15 \\
1.050 & 69 & 24 & 22 & 21 & 19 & 17 \\
1.075 & 113 & 28 & 26 & 24 & 22 & 19 \\
1.100 & 283 & 34 & 30 & 28 & 25 & 22 \\
1.125 & $\infty$ & 42 & 36 & 33 & 29 & 25 \\
1.150 & & 54 & 45 & 40 & 35 & 29 \\
1.175 & & 73 & 58 & 51 & 42 & 35 \\
1.200 & & 111 & 81 & 67 & 54 & 42 \\
1.225 & & 218 & 127 & 97 & 71 & 53 \\
1.250 & & 2583 & 279 & 166 & 104 & 70 \\
1.275 & & $\infty$ & $\infty$ & 520 & 184 & 100 \\
1.300 & & & & $\infty$ & 667 & 167 \\
1.325 & & & & & $\infty$ & 460 \\
1.350 & & & & & & $\infty$
\end{tabular}

Table 13: The New Zero Density Estimate 


\section{A Weighted Sum Over Primes $p \equiv a(\bmod q)$.}

In this section we take $a$ and $q$ coprime and investigate the sum

$$
\Sigma=\sum_{p \equiv a(\bmod q)} \frac{\log p}{p} f\left(\mathcal{L}^{-1} \log p\right)
$$

where the weight $f(x)$ is defined as

$$
f(x)=\left\{\begin{array}{rr}
0, & x \leq L-2 K \\
x-(L-2 K), & L-2 K \leq x \leq L-K, \\
L-x, & L-K \leq x \leq L, \\
0, & x \geq L
\end{array}\right.
$$

and $L, K$ are constants, satisfying $0<2 K<L$, which will be specified later. We modify $\Sigma$ so as to include prime powers. Since

$$
\sum_{n \geq N}, \frac{1}{n} \ll N^{-1 / 2}(\log N)^{-1}
$$

where $\sum^{\prime}$ denotes restriction to $n=p^{e}(e \geq 2)$, we find that

$$
\begin{aligned}
\Sigma & =\sum_{n \equiv a(\bmod q)} \frac{\Lambda(n)}{n} f\left(\mathcal{L}^{-1} \log n\right)+O\left(q^{-(L-2 K) / 2}\right) \\
& =\frac{1}{\phi(q)} \sum_{\chi(\bmod q)} \bar{\chi}(a) \sum_{n=1}^{\infty} \frac{\Lambda(n)}{n} \chi(n) f\left(\mathcal{L}^{-1} \log n\right)+O\left(q^{-(L-2 K) / 2}\right) .
\end{aligned}
$$

In the inner sum we replace $\chi$ by the primitive character $\chi^{*}$ which induces it. The error in so doing is

$$
\begin{aligned}
& \ll \sum_{p \mid q} \sum_{p^{e} \geq q^{L-2 K}} \frac{\log p}{p^{e}} \\
& \ll \sum_{p \mid q} \frac{\log p}{q^{L-2 K}} \\
& \ll \quad \mathcal{L} q^{-(L-2 K)} .
\end{aligned}
$$

For $\chi \neq \chi_{0}$ we have

$$
\mathcal{L}^{-1} \sum_{n=1}^{\infty} \frac{\Lambda(n)}{n} \chi^{*}(n) f\left(\mathcal{L}^{-1} \log n\right)=\frac{1}{2 \pi i} \int_{2-i \infty}^{2+i \infty}\left(-\frac{L^{\prime}}{L}\left(s, \chi^{*}\right)\right) F((1-s) \mathcal{L}) d s,
$$

where

$$
F(z)=e^{-(L-2 K) z}\left(\frac{1-e^{-K z}}{z}\right)^{2} .
$$


We shift the line of integration to $\Re s=-\frac{1}{2}$, and use the bounds

$$
\frac{L^{\prime}}{L}\left(s, \chi^{*}\right) \ll \log (q(1+|s|)), \quad F((1-s) \mathcal{L}) \ll q^{-3(L-2 K) / 2} \mathcal{L}^{-2}|s|^{-2},
$$

whence

$$
\frac{1}{2 \pi i} \int_{-\frac{1}{2}-i \infty}^{-\frac{1}{2}+i \infty}\left(-\frac{L^{\prime}}{L}\left(s, \chi^{*}\right)\right) F((1-s) \mathcal{L}) d s \ll \mathcal{L}^{-1} q^{-3(L-2 K) / 2} .
$$

It follows that

$$
\mathcal{L}^{-1} \sum_{n=1}^{\infty} \frac{\Lambda(n)}{n} \chi^{*}(n) f\left(\mathcal{L}^{-1} \log n\right)=-\sum_{\rho} F((1-\rho) \mathcal{L})+O\left(\mathcal{L}^{-1} q^{-3(L-2 K) / 2}\right),
$$

the sum on the right being over non-trivial zeros of $L\left(s, \chi^{*}\right)$. When $\chi=\chi_{0}$, the character $\chi^{*}$ is identically 1 , and

$$
\mathcal{L}^{-1} \sum_{n=1}^{\infty} \frac{\Lambda(n)}{n} f\left(\mathcal{L}^{-1} \log n\right)=F(0)+O\left(\mathcal{L}^{-1}\right)
$$

by partial summation. On comparing our various estimates we obtain

$$
\begin{aligned}
& \mathcal{L}^{-1} \Sigma=\frac{1}{\phi(q)}\left\{F(0)-\sum_{\chi \neq \chi_{0}} \bar{\chi}(a) \sum_{\rho} F((1-\rho) \mathcal{L})\right\} \\
&+O\left(\mathcal{L}^{-1} q^{-(L-2 K) / 2}\right)+O\left(q^{-(L-2 K)}\right)+O\left(\mathcal{L}^{-1} q^{-3(L-2 K) / 2}\right) \\
&+O\left(\mathcal{L}^{-1} \phi(q)^{-1}\right) .
\end{aligned}
$$

We therefore conclude as follows.

Lemma 13.1 Suppose that $L \geq 2 K+2$. Then, in the above notation, we have

$$
\left|\mathcal{L}^{-1} \Sigma-\frac{F(0)}{\phi(q)}\right| \leq \frac{1}{\phi(q)}\left\{\sum_{\chi \neq \chi_{0}} \sum_{\rho}|F((1-\rho) \mathcal{L})|+O\left(\mathcal{L}^{-1}\right)\right\} .
$$

In order to estimate the sum over zeros, we shall begin by using the bound (1.4), which implies that

$$
\sum_{\chi(\bmod q)} N(\sigma, T, \chi) \ll q^{3(1-\sigma)} T^{3 / 2} \quad(T \geq 1,0 \leq \sigma \leq 1),
$$

since the zeros are symmetrically distributed about $\sigma=\frac{1}{2}$. Thus in any rectangle

$$
1-\frac{m+1}{\mathcal{L}} \leq \beta \leq 1-\frac{m}{\mathcal{L}}, \quad \frac{1}{2} n \mathcal{L}^{-1} \leq|\gamma| \leq n \mathcal{L}^{-1},
$$


(where $n$ runs over powers of 2), there are

$$
\ll e^{3 m}\left(1+n \mathcal{L}^{-1}\right)^{3 / 2}
$$

zeros, each of which has

$$
F((1-\rho) \mathcal{L}) \ll e^{-(L-2 K) m} n^{-2} .
$$

If we sum over all rectangles (13.1) for which $\max (m, n) \geq R$ we obtain a total $O\left(R^{-2}\right)$, providing that $L>2 K+3$ and $1 \leq R \leq \mathcal{L}$. We now have

Lemma 13.2 Let $\varepsilon>0$ be given, and suppose that $L>2 K+3$. Then there exists an $R=R(\varepsilon)$, such that

$$
\left|\mathcal{L}^{-1} \Sigma-\frac{F(0)}{\phi(q)}\right| \leq \frac{1}{\phi(q)}\left\{\varepsilon+\sum_{\chi \neq \chi_{0}} \sum_{\rho}^{\prime}|F((1-\rho) \mathcal{L})|\right\}
$$

for sufficiently large $q$, where $\sum^{\prime}$ counts zeros in the rectangle

$$
1-\mathcal{L}^{-1} R \leq \beta \leq 1, \quad|\gamma| \leq \mathcal{L}^{-1} R
$$

In order to handle the remaining zeros we shall employ Lemma 5.2 with the function

$$
f_{1}(t)=\left\{\begin{array}{cc}
\sinh (K-t) \lambda, & 0 \leq t \leq K \\
0, & t \geq K
\end{array}\right.
$$

where $0<\lambda \leq R$. This yields

$$
F_{1}(z)=\int_{0}^{\infty} e^{-z t} f_{1}(t) d t=\frac{1}{2}\left\{\frac{e^{K \lambda}}{\lambda+z}+\frac{e^{-K \lambda}}{\lambda-z}-\frac{2 \lambda e^{-K z}}{\lambda^{2}-z^{2}}\right\}
$$

and

$$
\Re\left\{F_{1}(i t)\right\}=\frac{\lambda}{2} e^{K \lambda}\left|\frac{1-e^{-K(\lambda+i t)}}{\lambda+i t}\right|^{2}=\frac{\lambda}{2} e^{K \lambda}\left|F_{2}(\lambda+i t)\right| .
$$

where

$$
F_{2}(z)=e^{(L-2 K) z} F(z)=\left(\frac{1-e^{-K z}}{z}\right)^{2} .
$$

Thus

$$
\Re\left\{F_{1}(z)\right\} \geq \frac{\lambda}{2} e^{K \lambda}\left|F_{2}(\lambda+z)\right|
$$

for $\Re z=0$. Moreover $F_{1}(z)$ and $F_{2}(\lambda+z)$ tend uniformly to zero when $\Re z \geq 0$ and $|z| \rightarrow \infty$. It therefore follows from Lemma 4.1 that (13.2) holds for $\Re z \geq 0$.

For a particular character $\chi \neq \chi_{0}$, suppose that $L(s, \chi)$ has no zeros in the rectangle $1-\mathcal{L}^{-1} \lambda<\beta \leq 1,|\gamma| \leq 1$. Then (13.2) yields

$$
\sum_{\rho}^{\prime}\left|F_{2}((1-\rho) \mathcal{L})\right| \leq \frac{2}{\lambda} e^{-K \lambda} \sum_{\rho}^{\prime} \Re\left\{F_{1}((s-\rho) \mathcal{L})\right\},
$$


with $s=1-\mathcal{L}^{-1} \lambda$. Moreover, for any $\eta>0$, Lemmas 5.2 and 5.3 imply that

$$
\begin{aligned}
\sum_{\rho}{ }^{\prime} \Re\left\{F_{1}((s-\rho) \mathcal{L})\right\} & \leq f_{1}(0)\left(\frac{\phi}{2}+\eta\right)-\mathcal{L}^{-1} \sum_{n=1}^{\infty} \Lambda(n) \Re\left(\frac{\chi(n)}{n^{s}}\right) f_{1}\left(\mathcal{L}^{-1} \log n\right) \\
& \leq f_{1}(0)\left(\frac{\phi}{2}+\eta\right)+\mathcal{L}^{-1} \sum_{n=1}^{\infty} \Lambda(n) \frac{\chi_{0}(n)}{n^{s}} f_{1}\left(\mathcal{L}^{-1} \log n\right) \\
& \leq f_{1}(0)\left(\frac{\phi}{2}+\eta\right)+F_{1}((s-1) \mathcal{L})+\eta
\end{aligned}
$$

for $q \geq q(\eta)$. We conclude as follows.

Lemma 13.3 Let $\eta>0$ be given. Then

$$
\sum_{\rho}^{\prime}\left|F_{2}((1-\rho) \mathcal{L})\right| \leq \frac{\phi}{2}\left(\frac{1-e^{-2 K \lambda}}{\lambda}\right)+\frac{2 K \lambda-1+e^{-2 K \lambda}}{2 \lambda^{2}}+\eta
$$

for $q \geq q(\eta)$. In particular, taking $\lambda \rightarrow 0$, we obtain a bound $\phi K+K^{2}+\eta$.

When $K=1$ we obtain a bound $\phi+1+\eta \leq 1.333 \ldots$ This can be compared with the value 2.347 obtained by Chen [5; Lemma 6 ] for a certain subsum, using a quite different method. 


\section{Proof of Theorem 6; Siegel Zeros}

We begin our proof of Theorem 6 by considering the case in which $\lambda_{1}<0.348$, so that, according to Lemma $9.5, \rho_{1}$ must be real and simple, and $\chi_{1}$ must be real. We remark at the outset that we may suppose that $\lambda_{1} \gg 1$, for otherwise our result follows from the work of Heath-Brown [14], as described in $\S 1$. We shall use Lemmas 13.2 and 13.3 with $L=4.9$ and $K=0.25$. Since Table 7 gives $\lambda_{2} \geq 1.42$ when $\lambda_{1}<0.348$ we may take $\lambda=1.42$ and $\phi=\frac{1}{3}$ for $\chi \neq \chi_{1}$, and $\lambda=0$ and $\phi=\frac{1}{4}$ for $\chi=\chi_{1}$, giving bounds $0.10966 \ldots$ and 0.125 respectively, in Lemma 13.3. We have to treat $\rho_{1}$ separately, and we therefore specify the numbering system in $\S 11$ so that $\chi^{(1)}=\chi_{1}$,

$$
\Re\left(\rho^{(1)}\right)=\max \left\{\Re(\rho): L\left(\rho, \chi_{1}\right)=0,|\gamma| \leq 1, \rho \neq \rho_{1}\right\},
$$

and

$$
\Re\left(\rho^{(k)}\right)=\max \left\{\Re(\rho): L\left(\rho, \chi^{(k)}\right)=0,|\gamma| \leq 1\right\}
$$

for all $\chi^{(k)} \neq \chi_{1}$. Now, if we know, from Lemmas 8.3 and 8.7, or from Tables $2-7$, that $\lambda^{(k)} \geq \lambda$ for all $k$, then

$$
\begin{aligned}
\sum_{\chi \neq \chi_{0}} \sum_{\rho \neq \rho_{1}}{ }^{\prime}|F((1-\rho) \mathcal{L})| & \leq \sum_{\chi^{(k)} \neq \chi_{0}} e^{-(L-2 K) \lambda^{(k)}} \sum_{\rho}{ }^{\prime}\left|F_{2}((1-\rho) \mathcal{L})\right| \\
& \leq(0.10966 \ldots) \sum_{\chi^{(k)} \neq \chi_{0}, \chi_{1}} e^{-4.4 \lambda^{(k)}}+(0.125) e^{-4.4 \lambda^{(1)}} \\
& \leq(0.10966 \ldots) \sum_{k} e^{-4.4 \lambda^{(k)}}+(0.01533 \ldots) e^{-4.4 \lambda}
\end{aligned}
$$

We now observe that the function

$$
e^{-4.4 t}\left(\frac{e^{73 t / 30}-e^{16 t / 15}}{t}\right)
$$

is decreasing with respect to $t$, since $4.4>\frac{73}{30}>\frac{16}{15}$. Thus

$$
\begin{aligned}
\sum_{\lambda^{(k)} \geq \lambda} e^{-4.4 \lambda^{(k)}} & \leq e^{-4.4 \lambda}\left(\frac{e^{73 \lambda / 30}-e^{16 \lambda / 15}}{\lambda}\right) \sum_{k} \frac{\lambda^{(k)}}{\exp \left(\frac{73 \lambda^{(k)}}{30}\right)-\exp \left(\frac{16 \lambda^{(k)}}{15}\right)} \\
& \leq \frac{67}{6} e^{-4.4 \lambda}\left(\frac{e^{73 \lambda / 30}-e^{16 \lambda / 15}}{\lambda}\right)+\varepsilon,
\end{aligned}
$$

by Lemma 11.1. Since

$$
\frac{1-e^{-t}}{t} \leq 1
$$

for all $t>0$, we also have

$$
F\left(\left(1-\rho_{1}\right) \mathcal{L}\right)=K^{2}\left(\frac{1-e^{-\lambda_{1} K}}{\lambda_{1} K}\right)^{2} e^{-(L-2 K) \lambda_{1}}
$$




$$
\begin{aligned}
& \leq K^{2} e^{-(L-2 K) \lambda_{1}} \\
& =\frac{1}{16} e^{-4.4 \lambda_{1}} .
\end{aligned}
$$

Lemma 13.2 therefore implies the following.

Lemma 14.1 Let $\lambda_{1}<0.348$, and put $\lambda=\max \left(\lambda^{\prime}, \lambda_{2}\right)$. Suppose, in the notation of $\S 13$, that $L=4.9$ and $K=0.25$. Then

$$
\begin{gathered}
\mathcal{L}^{-1} \Sigma \geq \frac{1}{\phi(q)}\left\{\frac{1}{16}\left(1-e^{-4.4 \lambda_{1}}\right)-(0.10966 \ldots) \frac{67}{6} e^{-4.4 \lambda}\left(\frac{e^{73 \lambda / 30}-e^{16 \lambda / 15}}{\lambda}\right)\right. \\
\left.-(0.01533 \ldots) e^{-4.4 \lambda}-\varepsilon\right\}
\end{gathered}
$$

for $q \geq q(\varepsilon)$.

We wish to show that the expression on the right hand side above is positive for large $q$. Since $\lambda \geq 1.42$ we have

$$
(0.10966 \ldots) \frac{67}{6} e^{-4.4 \lambda}\left(\frac{e^{73 \lambda / 30}-e^{16 \lambda / 15}}{\lambda}\right) \leq(0.10966 \ldots) \frac{67}{6} \cdot \frac{\exp \left\{\left(\frac{70}{30}-4.4\right) \lambda\right\}}{1.42},
$$

and

$$
(0.01533 \ldots) e^{-4.4 \lambda} \leq(0.01533 \ldots) \exp \left\{-\frac{73}{30} 1.42\right\} \exp \left\{\left(\frac{73}{30}-4.4\right) \lambda\right\} .
$$

Together these total at most

$$
(0.86291 \ldots) \exp \left\{\left(\frac{73}{30}-4.4\right) \lambda\right\} \leq(0.86291 \ldots) e^{-(1.966 \ldots) \lambda} .
$$

However Lemmas 8.3 and 8.7 yield

$$
\lambda \geq\left(\frac{12}{11}-\varepsilon\right) \log \frac{1}{\lambda_{1}}
$$

and $\frac{12}{11} \times 1.966 \ldots>2$, so that Lemma 14.1 implies

$$
\mathcal{L}^{-1} \Sigma \geq \frac{1}{\phi(q)}\left\{\frac{1}{16}\left(1-e^{-4.4 \lambda_{1}}\right)-(0.86291 \ldots) \lambda_{1}^{2}\right\} .
$$

Since

$$
1-e^{-x} \geq x-\frac{x^{2}}{2}, \quad(x \geq 0),
$$

we therefore obtain

$$
\begin{aligned}
\mathcal{L}^{-1} \Sigma & \geq \frac{1}{\phi(q)}\left\{\frac{4.4}{16} \lambda_{1}-\frac{(4.4)^{2}}{32} \lambda_{1}^{2}-(0.86291 \ldots) \lambda_{1}^{2}\right\} \\
& =\frac{(1.46791 \ldots)}{\phi(q)} \lambda_{1}\left\{0.18734 \ldots-\lambda_{1}\right\}
\end{aligned}
$$


so that $\Sigma>0$ whenever $\lambda_{1} \leq 0.187$, providing that $q$ is large enough.

We break the remaining range into two subintervals $[0.187,0.3]$ and $[0.3,0.348]$. On the first interval we have $\lambda \geq 1.58$, by Tables 3 and 7 . Moreover, the lower bound in Lemma 14.1 is increasing with respect to both $\lambda_{1}$ and $\lambda$. Thus it suffices to check that one obtains a positive bound with $\lambda_{1}=0.187$ and $\lambda=1.58$. Similarly to cover the range [0.3,0.348] it suffices to check with $\lambda_{1}=0.3$ and $\lambda=1.42$. For each range a calculation shows that the lower bound obtained is indeed positive, and we conclude as follows.

Lemma 14.2 When $L=4.9, K=0.25$ and $\lambda_{1}<0.348$ we have $\Sigma \gg \mathcal{L} / \phi(q)$ for sufficiently large $q$.

Thus Linnik's constant may be taken as 4.9 in the 'Siegel Zero' case. 


\section{Proof of Theorem 6; Large Values of $\lambda_{1}$}

In this section we suppose that $\lambda_{1} \geq 0.348$. As before, the zero $\rho_{1}$ will receive special treatment. However this time we number the characters using (14.1) for all $k$. For $\chi=\chi_{k}$ with $k \geq 2$, Lemma 13.3 yields

$$
\begin{aligned}
K^{-2} \sum_{\rho}{ }^{\prime}|F((1-\rho) \mathcal{L})| & \leq e^{-(L-2 K) \lambda_{k}} K^{-2} \sum_{\rho}^{\prime}\left|F_{2}((1-\rho) \mathcal{L})\right| \\
& \leq e^{-(L-2 K) \lambda_{k}}\left(B\left(\lambda_{k}\right)+\eta\right),
\end{aligned}
$$

where

$$
B(\lambda)=\left(\frac{1-e^{-2 K \lambda}}{6 K^{2} \lambda}\right)+\frac{2 K \lambda-1+e^{-2 K \lambda}}{2 K^{2} \lambda^{2}} .
$$

For $\chi=\chi_{1}$ with $\chi_{1}$ complex, we obtain

$$
\begin{aligned}
& K^{-2} \sum_{\rho}{ }^{\prime}|F((1-\rho) \mathcal{L})| \\
\leq & K^{-2}\left\{e^{-(L-2 K) \lambda_{1}}\left|F_{2}\left(\left(1-\rho_{1}\right) \mathcal{L}\right)\right|+e^{-(L-2 K) \lambda^{\prime}} \sum_{\rho \neq \rho_{1}}^{\prime}\left|F_{2}((1-\rho) \mathcal{L})\right|\right\} \\
= & K^{-2}\left\{\left(e^{-(L-2 K) \lambda_{1}}-e^{-(L-2 K) \lambda^{\prime}}\right)\left|F_{2}\left(\left(1-\rho_{1}\right) \mathcal{L}\right)\right|\right. \\
& \left.+e^{-(L-2 K) \lambda^{\prime}} \sum_{\rho}^{\prime}\left|F_{2}((1-\rho) \mathcal{L})\right|\right\} \\
\leq & K^{-2}\left(e^{-(L-2 K) \lambda_{1}}-e^{-(L-2 K) \lambda^{\prime}}\right) F_{2}\left(\lambda_{1}\right)+e^{-(L-2 K) \lambda^{\prime}}\left(B\left(\lambda_{1}\right)+\eta\right) \\
\leq & e^{-(L-2 K) \lambda_{1}}\left(B\left(\lambda_{1}\right)+\eta\right) \quad-\left(e^{-(L-2 K) \lambda_{1}}-e^{-(L-2 K) \lambda^{\prime}}\right)\left(B\left(\lambda_{1}\right)-K^{-2} F_{2}\left(\lambda_{1}\right)\right) .
\end{aligned}
$$

If $\chi_{1}$ is real and $\rho_{1}$ is also real we get the same result, but if $\chi_{1}$ is real and $\rho_{1}$ is complex we obtain

$$
e^{-(L-2 K) \lambda_{1}}\left(B\left(\lambda_{1}\right)+\eta\right)-\left(e^{-(L-2 K) \lambda_{1}}-e^{-(L-2 K) \lambda^{\prime}}\right)\left(B\left(\lambda_{1}\right)-2 K^{-2} F_{2}\left(\lambda_{1}\right)\right) .
$$

We therefore write

$$
A=A_{1}=\left(e^{-(L-2 K) \lambda_{1}}-e^{-(L-2 K) \lambda^{\prime}}\right)\left(B\left(\lambda_{1}\right)-K^{-2} F_{2}\left(\lambda_{1}\right)\right),
$$

if $\chi_{1}$ is complex, or $\chi_{1}$ and $\rho_{1}$ are real; and

$$
A=A_{2}=\left(e^{-(L-2 K) \lambda_{1}}-e^{-(L-2 K) \lambda^{\prime}}\right)\left(B\left(\lambda_{1}\right)-2 K^{-2} F_{2}\left(\lambda_{1}\right)\right),
$$

if $\chi_{1}$ is real and $\rho_{1}$ is complex. If we now write $n(\chi)=1$ or 2 according as $\chi$ is real or complex, we obtain

$$
K^{-2} \sum_{\chi \neq \chi_{0}} \sum_{\rho}^{\prime}|F((1-\rho) \mathcal{L})| \leq \varepsilon+\sum_{k} e^{-(L-2 K) \lambda^{(k)}} B\left(\lambda^{(k)}\right)-n\left(\chi_{1}\right) A .
$$


We shall compare the sum on the right with that occuring in Lemma 11.1. We put

$$
w(\lambda)=\lambda /\left\{e^{73 \lambda / 30}-e^{16 \lambda / 15}\right\}
$$

so that

$$
\sum_{k} w\left(\lambda^{(k)}\right) \leq \frac{67}{6}+\varepsilon .
$$

For small values of $\lambda^{(k)}$ the work of $\$ 12$, and in particular the bounds given by Table 13, are more powerful than what is implied by Lemma 11.1. We therefore choose an appropriate constant $\Lambda$, and treat the cases $\lambda^{(k)}>\Lambda$ and $\lambda^{(k)} \leq \Lambda$ separately. We begin by observing that $e^{-(L-2 K) \lambda} B(\lambda) / w(\lambda)$ is a decreasing function of $\lambda$ when $L-2 K>\frac{73}{30}$. To check this it suffices to show that each of the functions

$$
\frac{1-e^{-x}}{x}, \quad \frac{x-1+e^{-x}}{x^{2}}, \quad \text { and } \quad \frac{e^{-a x}-e^{-b x}}{x} \quad(0<a<b)
$$

is decreasing. It follows that

$$
e^{-(L-2 K) \lambda^{(k)}} B\left(\lambda^{(k)}\right) \leq e^{-(L-2 K) \Lambda} B(\Lambda) w(\Lambda)^{-1} w\left(\lambda^{(k)}\right) \quad\left(\lambda^{(k)}>\Lambda\right),
$$

whence

$$
\begin{aligned}
\sum_{k} e^{-(L-2 K) \lambda^{(k)}} B\left(\lambda^{(k)}\right) & \\
\leq & e^{-(L-2 K) \Lambda} B(\Lambda) w(\Lambda)^{-1} \sum_{\lambda^{(k)}>\Lambda} w\left(\lambda^{(k)}\right) \\
& +\sum_{\lambda^{(k)} \leq \Lambda} e^{-(L-2 K) \lambda^{(k)}} B\left(\lambda^{(k)}\right)
\end{aligned}
$$

We now write

$$
C(\lambda)=e^{-(L-2 K) \lambda} B(\lambda)-e^{-(L-2 K) \Lambda} B(\Lambda) \frac{w(\lambda)}{w(\Lambda)},
$$

and we conclude from (15.1) and (15.2) that

$$
\begin{aligned}
& K^{-2} \sum_{\chi \neq \chi_{0}} \sum_{\rho}{ }^{\prime}|F((1-\rho) \mathcal{L})| \\
& \quad \leq \varepsilon+\frac{67}{6} e^{-(L-2 K) \Lambda} \frac{B(\Lambda)}{w(\Lambda)}+\sum_{\lambda^{(k)} \leq \Lambda} C\left(\lambda^{(k)}\right)-n\left(\chi_{1}\right) A
\end{aligned}
$$


with a new value of $\varepsilon$. We may now observe that

$$
C(\lambda)=w(\lambda)\left\{e^{-(L-2 K) \lambda} \frac{B(\lambda)}{w(\lambda)}-e^{-(L-2 K) \Lambda} \frac{B(\Lambda)}{w(\Lambda)}\right\}
$$

which is the product of two non-negative decreasing functions on $[0, \Lambda]$, and so is itself non-negative and decreasing.

To bound $\sum C\left(\lambda^{(k)}\right)$ we separate off the terms with $\lambda^{(k)} \leq \lambda_{2}$, giving

$$
n\left(\chi_{1}\right) C\left(\lambda_{1}\right)+n\left(\chi_{2}\right) C\left(\lambda_{2}\right)+\sum_{\lambda_{3}^{*} \leq \lambda^{(k)} \leq \Lambda} C\left(\lambda^{(k)}\right),
$$

where $\lambda_{3}^{*}=\min \left(\lambda_{3}, \Lambda\right)$. To estimate the final sum we shall use the bounds for $N(\lambda)$ given by Table 13. We therefore write $\Lambda_{r}=\Lambda-(0.025) r$, and define $s$ so that $\Lambda_{s+1}<\lambda_{3}^{*} \leq \Lambda_{s}$. Then

$$
\begin{aligned}
\sum_{\lambda_{3}^{*} \leq \lambda^{(k)} \leq \Lambda} C\left(\lambda^{(k)}\right)= & \sum_{\substack{\lambda_{3}^{*} \leq \lambda^{(k)} \leq \Lambda_{s} \\
\leq}} C\left(\lambda^{(k)}\right)+\sum_{r=0}^{s-1} \sum_{\Lambda_{r+1}<\lambda^{(k)} \leq \Lambda_{r}} C\left(\lambda^{(k)}\right) \\
& +\sum_{r=0}^{s-1}\left\{N\left(\Lambda_{s}\right)-n\left(\chi_{1}\right)-n\left(\chi_{2}\right)\right\} C\left(\lambda_{3}^{*}\right)
\end{aligned}
$$

since $C(\lambda)$ is decreasing. Abel's identity now shows that the above bound is

$$
\begin{array}{r}
-\left(n\left(\chi_{1}\right)+n\left(\chi_{2}\right)\right) C\left(\lambda_{3}^{*}\right)+N\left(\Lambda_{s}\right)\left\{C\left(\lambda_{3}^{*}\right)-C\left(\Lambda_{s}\right)\right\} \\
+\sum_{r=0}^{s-1} N\left(\Lambda_{r}\right)\left\{C\left(\Lambda_{r+1}\right)-C\left(\Lambda_{r}\right)\right\}
\end{array}
$$

on recalling that $C\left(\Lambda_{0}\right)=0$. Here we may replace $N(\lambda)$ by its upper bound, $N_{0}(\lambda)$, say, in Table 13 . In order to do this we must observe that $C\left(\lambda_{3}^{*}\right) \geq C\left(\Lambda_{s}\right)$ and $C\left(\Lambda_{r+1}\right) \geq C\left(\Lambda_{r}\right)$. We then have

$$
\begin{aligned}
\sum_{\lambda_{3}^{*} \leq \lambda^{(k)} \leq \Lambda} C\left(\lambda^{(k)}\right) \leq & -\left(n\left(\chi_{1}\right)+n\left(\chi_{2}\right)\right) C\left(\lambda_{3}^{*}\right)+N_{0}\left(\Lambda_{s}\right)\left\{C\left(\lambda_{3}^{*}\right)-C\left(\Lambda_{s}\right)\right\} \\
& +\sum_{r=0}^{s-1} N_{0}\left(\Lambda_{r}\right)\left\{C\left(\Lambda_{r+1}\right)-C\left(\Lambda_{r}\right)\right\} \\
= & \left\{N_{0}\left(\Lambda_{s}\right)-n\left(\chi_{1}\right)-n\left(\chi_{2}\right)\right\} C\left(\lambda_{3}^{*}\right) \\
& +\sum_{r=0}^{s-1}\left\{N_{0}\left(\Lambda_{r}\right)-N_{0}\left(\Lambda_{r+1}\right)\right\} C\left(\Lambda_{r+1}\right) .
\end{aligned}
$$


It now follows that

$$
\begin{aligned}
& \sum_{\lambda^{(k)} \leq \Lambda} C\left(\lambda^{(k)}\right) \\
& \leq n\left(\chi_{1}\right) C\left(\lambda_{1}\right)+n\left(\chi_{2}\right) C\left(\lambda_{2}\right)+\left\{N_{0}\left(\Lambda_{s}\right)-n\left(\chi_{1}\right)-n\left(\chi_{2}\right)\right\} C\left(\lambda_{3}^{*}\right) \\
& +\sum_{r=0}^{s-1}\left\{N_{0}\left(\Lambda_{r}\right)-N_{0}\left(\Lambda_{r+1}\right)\right\} C\left(\Lambda_{r+1}\right) \\
& =n\left(\chi_{1}\right)\left\{C\left(\lambda_{1}\right)-C\left(\lambda_{3}^{*}\right)\right\}+n\left(\chi_{2}\right)\left\{C\left(\lambda_{2}\right)-C\left(\lambda_{3}^{*}\right)\right\}+N_{0}\left(\Lambda_{s}\right) C\left(\lambda_{3}^{*}\right) \\
& +\sum_{r=0}^{s-1}\left\{N_{0}\left(\Lambda_{r}\right)-N_{0}\left(\Lambda_{r+1}\right)\right\} C\left(\Lambda_{r+1}\right) .
\end{aligned}
$$

On comparing this with (15.3) we see that $n\left(\chi_{2}\right)$ may be replaced by 2 , since $C\left(\lambda_{2}\right) \geq C\left(\lambda_{3}^{*}\right)$. Moreover, when $\chi_{1}$ is real, we may replace the terms

$$
n\left(\chi_{1}\right)\left(C\left(\lambda_{1}\right)-C\left(\lambda_{3}^{*}\right)-A\right)
$$

by

$$
2\left(C\left(\lambda_{1}\right)-C\left(\lambda_{3}^{*}\right)-A_{1}\right)
$$

with a loss

$$
T=\left\{\begin{array}{cc}
C\left(\lambda_{3}^{*}\right)-C\left(\lambda_{1}\right)+A_{1}, & \rho_{1} \text { real } \\
C\left(\lambda_{3}^{*}\right)-C\left(\lambda_{1}\right)+2 A_{1}-A_{2}, & \rho_{1} \text { complex }
\end{array}\right.
$$

For $\chi_{1}$ complex we shall take $T=0$. We conclude as follows.

Lemma 15.1 Let $\lambda_{1} \geq 0.384$. Then, in the above notation, we have

$$
\begin{aligned}
K^{-2} \sum_{\chi \neq \chi_{0}} \sum_{\rho} & |F((1-\rho) \mathcal{L})| \\
\leq & \varepsilon+\frac{67}{6} e^{-(L-2 K) \Lambda} \frac{B(\Lambda)}{w(\Lambda)}+2\left(C\left(\lambda_{1}\right)-A\right)+2 C\left(\lambda_{2}\right) \\
& +\left\{N_{0}\left(\Lambda_{s}\right)-4\right\} C\left(\lambda_{3}^{*}\right)+\sum_{r=0}^{s-1}\left\{N_{0}\left(\Lambda_{r}\right)-N_{0}\left(\Lambda_{r+1}\right)\right\} C\left(\Lambda_{r+1}\right)+T,
\end{aligned}
$$

where $T$ is given by (15.4).

We now calculate the right hand side above, taking $L=5.5$ and $K=0.3$. For the range $0.348 \leq \lambda_{1} \leq 0.4$ we use the bounds for $N_{0}\left(\Lambda_{r}\right)$ given in the second column of Table 13. We take $\Lambda=1.2$, since a comparison with Table 12 shows that (12.1) is superior for $\lambda \geq 1.2$. When $0.348 \leq \lambda_{1} \leq 0.36$ we use the estimates $\lambda^{\prime} \geq 1.309, \lambda_{2} \geq 0.903$ and $\lambda_{3}^{*} \geq 1.2$ given by Tables 8,10 and 9 
respectively. To handle the term $T$ we may observe that the case $\chi_{1}$ real, $\rho_{1}$ complex, arises only for $\lambda_{1} \geq 0.5$, by Lemma 9.5. We find that

$$
\begin{aligned}
& 2\left(C\left(\lambda_{1}\right)-A\right) \leq 0.2874 \ldots \\
& 2 C\left(\lambda_{2}\right) \leq 0.02504 \ldots \\
&\left\{N_{0}\left(\Lambda_{s}\right)-4\right\} C\left(\lambda_{3}^{*}\right)+\sum_{r=0}^{s-1}\left\{N_{0}\left(\Lambda_{r}\right)-N_{0}\left(\Lambda_{r+1}\right)\right\} C\left(\Lambda_{r+1}\right)=0 \\
& \frac{67}{6} e^{-(L-2 K) \Lambda} \frac{B(\Lambda)}{w(\Lambda)} \leq 0.6178 \ldots \\
& T \leq-0.1437 \ldots \text { (for } \chi_{1} \text { real). }
\end{aligned}
$$

The first four of these may be thought of as the contributions from, respectively:$\chi_{1}\left(\right.$ and $\left.\overline{\chi_{1}}\right) ; \chi_{2}\left(\right.$ and $\left.\overline{\chi_{2}}\right) ; \chi_{j}\left(\right.$ and $\bar{\chi}_{j}$ ) with $j \geq 3$ and $\lambda_{j} \leq \Lambda$; and the rest. Here the total is at most $0.9304 \ldots<1$, so that

$$
\Sigma \gg \frac{\mathcal{L}}{\phi(q)}
$$

in this case.

When $0.36 \leq \lambda_{1} \leq 0.38$ we use the bounds $\lambda^{\prime} \geq 1.25, \lambda_{2} \geq 0.887, \lambda_{3} \geq 1.181$ from Tables 8,10 and 9, and we obtain a total contribution of at most $0.9465 \ldots$ Similarly for $0.38 \leq \lambda_{1} \leq 0.4$ we obtain a total of at most $0.9804 \ldots$ Over the range $[0.4,0.44]$ we use the third column of Table 13, taking $\Lambda=1.225$, and considering the cases $0.4 \leq \lambda_{1} \leq 0.42$ and $0.42 \leq \lambda_{1} \leq 0.44$ separately. For the intervals $0.44 \leq \lambda_{1} \leq 0.5,0.5 \leq \lambda_{1} \leq 0.58$ and $0.58 \leq$ $\lambda_{1} \leq 0.6$ we apply analogous arguments, taking $\Lambda=1.225,1.25$ and 1.3 respectively, and splitting the ranges for $\lambda_{1}$ into subintervals of length 0.02 . (Note however that for $0.58 \leq \lambda_{1} \leq 0.6$ we use the estimate $\lambda_{3} \geq 0.857$ given in Lemma 10.3, rather than the bound given in Table 9.) For example, when $0.48 \leq \lambda_{1} \leq 0.5$, the figures corresponding to the bounds (15.5)(15.9) are $0.1502 \ldots, 0.0505 \ldots, 0.2230 \ldots, 0.5705 \ldots$, and $-0.0658 \ldots$, with total $0.9943 \ldots$ in the case $\chi_{1}$ complex. For $0.58 \leq \lambda_{1} \leq 0.6$ we find that $T \leq 0$ when $\chi_{1}$ is real, whether $\rho_{1}$ is real or not. In fact $T$ is largest $(0.00757 \ldots)$ on the interval $[0.5,0.52]$, while the remaining terms in Lemma 15.1 are largest $(0.9908 \ldots)$ on $[0.56,0.58]$. Finally when $\lambda_{1} \geq 0.6$, we may use the universal bounds $\lambda^{\prime} \geq 0.696, \lambda_{2} \geq 0.702$ and $\lambda_{3} \geq 0.857$ of Table 8 and Lemmas 9.4 and 10.3 , to get figures $0.1336 \ldots, 0.0975 \ldots, 0.3111 \ldots$ and $0.4493 \ldots$ for $(15.5)-$ (15.8), with total $0.9917 \ldots$ In this range too we have $T \leq 0$ in every case. We have now verified that (15.10) holds whenever $\lambda_{1} \geq 0.348$. This completes the proof of Theorem 6 . 


\section{Small Improvements}

There are quite a number of places where the foregoing arguments could be subject to small improvements. The purpose of this final section is to mention some of these.

1) As has been observed, our description of the functions $f$ and $F$ of $\S 7$ as "optimal" leaves something to be desired. It should in principle be possible to optimize, say

$$
\left(F\left(-\lambda_{1}\right)-F\left(\lambda_{2}-\lambda_{1}\right)-F(0)\right) / f(0) .
$$

One would be lead to a differential equation of order 4 in place of (7.10). The solution of this would lead to functions $f$ which should be optimal in this wider setting.

2) It will be apparent from the work of $\S 10$, and in particular our treatment of (10.8) and (10.12), that similar arguments might be tried in each case where we have used the polynomial $P_{3}$ in place of a function $f$. For example, in the proof of Lemma 9.5 for the case $h=4$, one would consider the same function $F$ as one uses for $h=5$, and investigate

$$
\max _{t \in \mathbb{R}} \Re\left\{1250 F\left(-\lambda_{1}+i t\right)-6000 F(i t)\right\} .
$$

3) Another technique applicable at a number of points hinges on the fact that the functions $f$ in Lemma 5.2 which occur in practice are positive on a range strictly larger than $[0,1]$. Indeed, when one uses the "polynomial method" of $\S 4$, the corresponding functions are positive throughout $[0, \infty)$. The significance of the constant 1 is that we apply the Brun-Titchmarsh Theorem in the form

$$
\pi(x ; q, a) \ll \frac{x}{\phi(q) \log (x / q)}
$$

for $x \geq 2 q$. This is non-trivial over at least part of the range in which $f$ is strictly positive.

We illustrate the method in connection with Lemma 6.2, and suppose for simplicity that $\gamma_{1}=\gamma_{2}=0$. Moreover we shall prove only very weak bounds. However this should be enough to indicate that some improvement, albeit a small one, is possible.

From (16.1), and from our hypothesis about the function $f$, it follows that there are constants $c_{1}>c_{2}>1$ and $c_{3}, c_{4}>0$ such that $f(x) \geq c_{3}$ on $\left[c_{2}, c_{1}\right]$, and

$$
\pi(x ; q, a) \leq c_{4} \frac{x}{\phi(q) \log x}, \quad \text { for } \quad x \geq q^{c_{2}} .
$$

Since $\gamma_{1}=\gamma_{2}=0$ the inequality (6.3) may be strengthened to yield

$$
\left\{\chi_{0}(n)+\Re\left(\chi(n) n^{-i \gamma_{1}}\right)\right\}\left\{\chi_{0}(n)+\Re\left(\chi_{2}(n) n^{-i \gamma_{2}}\right)\right\} \geq(1-\cos \theta)^{2} \chi_{0}(n),
$$


except when $\arg \chi_{1}(n)$ or $\arg \chi_{2}(n)$ lies in the interval $[\pi-\theta, \pi+\theta]$. Thus, instead of merely taking 0 on the right in (6.4), we may put

$$
c_{3}(1-\cos \theta)^{2}\left\{\Sigma^{(0)}-\Sigma^{(1)}\right\} \Lambda(n) n^{-\beta_{1}} .
$$

Here $\Sigma^{(0)}$ runs over integers $n$ in the range

$$
q^{c_{2}} \leq n \leq q^{c_{1}}
$$

subject to $(q, n)=1$, whereas $\Sigma^{(1)}$ runs over the same $n$ but restricted to those residue classes for which either $\arg \chi_{1}(n)$ or $\arg \chi_{2}(n)$ is in $[\pi-\theta, \pi+\theta]$.

Our aim is to show that

$$
\left\{\Sigma^{(0)}-\Sigma^{(1)}\right\} \Lambda(n) n^{-\beta_{1}} \gg \mathcal{L},
$$

if $\theta$ is chosen to be a suitably small constant. Such an inequality automatically leads to an improvement in any application of Lemma 6.2. We may of course assume that $\beta_{1}=1+O\left(\mathcal{L}^{-1}\right)$, whence

$$
n^{-\beta_{1}} \gg n^{-1}
$$

for the range of $n$ in question. Moreover we have

$$
\Sigma^{(0)} \Lambda(n) n^{-1} \sim\left(c_{1}-c_{2}\right) \mathcal{L}
$$

independently of $\theta$. It therefore remains to bound

$$
\Sigma^{(1)} \Lambda(n) n^{-1} .
$$

We shall assume for simplicity that both $\chi_{1}$ and $\chi_{2}$ have large order. There are then at most $\theta \phi(q)$ residue classes modulo $q$ occuring in the sum $\Sigma^{(1)}$. For each such class (16.1) yields

$$
\begin{aligned}
\sum_{n \equiv a(\bmod q)}(1) \frac{\Lambda(n)}{n}= & \left(\pi\left(q^{c_{1}} ; q, a\right)-\pi\left(q^{c_{2}} ; q, a\right)\right) \frac{\log q^{c_{1}}}{q^{c_{1}}} \\
& +\int_{q^{c_{2}}}^{q^{c_{1}}}\left(\pi(x ; q, a)-\pi\left(q^{c_{2}} ; q, a\right)\right) \frac{\log x-1}{x^{2}} d x+O\left(\phi(q)^{-1}\right) \\
\leq & \int_{q^{c_{2}}}^{q^{c_{1}}} \pi(x ; q, a) \frac{\log x}{x^{2}} d x+O\left(\phi(q)^{-1}\right) \\
\leq & \frac{c_{4}}{\phi(q)} \int_{q^{c_{2}}}^{q^{c_{1}}} \frac{d x}{x}+O\left(\phi(q)^{-1}\right) \\
= & \frac{c_{4}\left(c_{1}-c_{2}\right) \mathcal{L}}{\phi(q)}+O\left(\phi(q)^{-1}\right)
\end{aligned}
$$


by partial summation. It now follows that

$$
\Sigma^{(1)} \frac{\Lambda(n)}{n} \leq \theta c_{4}\left(c_{1}-c_{2}\right) \mathcal{L}+O(1) .
$$

We may therefore use (16.3) and (16.4) to deduce (16.2), on taking $\theta=1 /\left(2 c_{4}\right)$, say.

4) An improvement can be made in $\S 8$, when one is estimating $\lambda_{2}$ in the case in which $\phi\left(\chi_{2}\right)=\frac{1}{3}$. This is done by sharpening the bound $\psi \leq \frac{11}{24}$ to $\psi \leq \frac{97}{216}$.

Suppose that $q=\prod p^{e}$, and let

$$
q_{1}=\prod_{p \mid q} p, \quad v=\prod_{p^{e} \| q, e \geq 3} p^{e}, \quad q_{2}=v^{3} q .
$$

It is the apparent from the work of $\S 2$, and from Lemma 2.3 in particular, that the bound on the right of (2.5) may be replaced by

$$
q_{1}^{\frac{1}{4}(1-\sigma)\left(1+k^{-1}\right)+\varepsilon}(1+|t|)
$$

for $\chi$ real, and either

$$
q^{\frac{1}{3}(1-\sigma)\left(1+k^{-1}\right)+\varepsilon}(1+|t|)
$$

or

$$
q_{2}^{\frac{1}{4}(1-\sigma)\left(1+k^{-1}\right)+\varepsilon}(1+|t|)
$$

in general. Thus we may estimate $\psi$ as

$$
\frac{1}{8} \cdot \frac{\log q_{1}}{\log q}+\min \left(\frac{1}{3}, \frac{1}{4} \cdot \frac{\log q_{2}}{\log q}\right) .
$$

If $q_{2} \geq q^{4 / 3}$ we use the inequality

$$
27 \leq\left\{\begin{array}{cc}
25 e+18 \frac{e}{9}, & e=1,2 \\
25 e+18\left(\frac{e}{9}-e\right), & e \geq 3
\end{array}\right.
$$

whence

$$
q_{1}^{27} \leq q^{25}\left(\frac{q^{1 / 9}}{v}\right)^{18}=q^{25}\left(\frac{q^{4 / 3}}{q_{2}}\right)^{6} \leq q^{25} .
$$

In this case we have

$$
\psi \leq \frac{1}{8} \cdot \frac{25}{27}+\frac{1}{3}=\frac{97}{216} .
$$

On the other hand, if $q_{2} \leq q^{4 / 3}$, we use the inequalities

$$
\begin{aligned}
27 & \leq 43 e+144\left(-\frac{e}{9}\right), \quad e=1,2, \\
27(1+6 e) & \leq 43 e+144\left(e-\frac{e}{9}\right), \quad e \geq 3,
\end{aligned}
$$


whence

$$
\left(q_{1} q_{2}^{2}\right)^{27}=\left(q_{1} v^{6}\right)^{27} q^{54} \leq q^{43}\left(\frac{v}{q^{1 / 9}}\right)^{144} q^{54}=q^{97}\left(\frac{q_{2}}{q^{4 / 3}}\right)^{48} \leq q^{97} .
$$

In this case therefore we also have

$$
\psi \leq \frac{1}{8} \cdot \frac{\log q_{1} q_{2}^{2}}{\log q} \leq \frac{1}{8} \cdot \frac{97}{27}=\frac{97}{216},
$$

as required.

5) We can improve on Lemmas 9.2 and 9.3 if we impose further restrictions on $\chi_{j}$. Thus, in Lemma 9.2 for example, we may replace $K\left(\beta_{1}+i t, \chi\right)$ in the proof by $K\left(\beta^{*}+i t, \chi\right)$, where

$$
\beta^{*}=1-\mathcal{L}^{-1} \min \left(\lambda^{\prime}, \lambda_{2}\right) .
$$

This requires that none of $\chi_{1} \chi_{j}, \overline{\chi_{1}} \chi_{j}, \chi_{1}^{2} \chi_{j}, \chi_{1}^{2}$ or $\bar{\chi}_{1}^{2} \chi_{j}$ should equal any of $\chi_{0}, \chi_{1}$ or $\overline{\chi_{1}}$. If $\chi_{1}$ has order 4 or more this means that

$$
\chi_{j} \neq \chi_{1}^{2},{\overline{\chi_{1}}}^{2}, \chi_{1}^{3} \text {, or }{\overline{\chi_{1}}}^{3} .
$$

Thus at least one of $\chi_{2}, \chi_{3}$ or $\chi_{4}$ is admissable, and we obtain a bound for $\lambda_{4}$.

6 ) It is possible to strengthen the argument of $\S 11$ by replacing $b_{n}$ in (11.12) by

$$
b_{n}^{\prime}=\left\{\begin{array}{cc}
b_{n}, & \text { if } \sum_{d \mid n} \theta_{d} \neq 0 \\
0, & \text { otherwise }
\end{array}\right.
$$

This results in an improvement in (11.14) of

$$
\sum_{n=1}^{\infty} '\left(\sum_{d \mid n} \psi_{d}\right)^{2} n^{-1}\left(e^{-n / X}-e^{-n \mathcal{L}^{2} / U}\right),
$$

where $\Sigma^{\prime}$ indicates the condition

$$
\sum_{d \mid n} \theta_{d}=0
$$

To demonstrate that a saving is indeed achieved, we restrict attention to integers $n=p_{1} p_{2} p_{3}$ where the $p_{i}$ are primes in the ranges

$$
W^{1 / 3}<p_{1}<W^{2 / 5}<p_{2}<W^{1 / 2}, \quad U W^{-1 / 3}<p_{3}<U W^{-1 / 4} .
$$

Note that the choice of parameters made in practice ensures that $p_{3}>W$ and that $p_{1} p_{2} p_{3}<V<X$. We now find from the definitions (11.6) and (11.7) that (16.6) holds and that

$$
\sum_{d \mid n} \psi_{d}=\frac{\log U / p_{3}}{\log V / U} \gg 1
$$


Since

$$
e^{-n / X}-e^{-n \mathcal{L}^{2} / U} \gg 1
$$

for our values of $n$, the contribution to (16.5) is therefore

$$
\gg \sum\left(p_{1} p_{2} p_{3}\right)^{-1} \gg\left(\sum_{\gg 1} p_{1}^{-1}\right)\left(\sum p_{2}^{-1}\right)\left(\sum p_{3}^{-1}\right)
$$

as required.

7) A second way of improving the argument of $\S 11$ uses a rather better choice of the coefficients $a_{n \chi}$ and $b_{n}$ in (11.11) and (11.12). We use a continuous monotonic function $w(x)$ satisfying $1 \ll w(x) \ll 1$, and we replace $a_{n \chi}$ and $b_{n}$ by

$$
w\left(\mathcal{L}^{-1} \log n\right) a_{n \chi}, \quad w\left(\mathcal{L}^{-1} \log n\right)^{-1} b_{n}
$$

respectively. Instead of (11.14) we now have

$$
\begin{aligned}
\sum_{n=1}^{\infty} w\left(\mathcal{L}^{-1}\right. & \log n)^{-2}\left(\sum_{d \mid n} \psi_{d}\right)^{2} n^{-1}\left(e^{-n / X}-e^{-n \mathcal{L}^{2} / U}\right) \\
= & \frac{1+O\left(\mathcal{L}^{-1}\right)}{(v-u)^{2}} \int_{u}^{x} w(t)^{-2} \min (t-u, v-u) d t
\end{aligned}
$$

The non-diagonal terms still make a negligible contribution to (11.15), while the typical diagonal term is now

$$
\begin{array}{r}
\left|C_{\chi}\right|^{2} w_{\chi} \sum_{n=1}^{\infty} w\left(\mathcal{L}^{-1} \log n\right)^{2}\left(\sum_{d \mid n} \theta_{d}\right)^{2} \chi_{0}(n) n^{1-2 \beta}\left(e^{-n / X}-e^{-n \mathcal{L}^{2} / U}\right) \\
=\frac{1+O\left(\mathcal{L}^{-1} \log \mathcal{L}\right)}{w} \int_{u}^{x} w(t)^{2} e^{2 \mathcal{L}(1-\beta) t} d t
\end{array}
$$

In order to make the optimal choice of the function $w(t)$ we shall consider only zeros with $\beta \geq 1-\mathcal{L}^{-1} \lambda$. Taking $w_{\chi}=1$ for the corresponding characters, we obtain

$$
N(\lambda) \leq \frac{1+o(1)}{(v-u)^{2} w}\left\{\int_{u}^{x} w(t)^{-2} \min (t-u, v-u) d t\right\}\left\{\int_{u}^{x} w(t)^{2} e^{2 \lambda t} d t\right\} .
$$

The optimal choice is now

$$
w(t)=e^{-\lambda t / 2}\{\min (t-u, v-u)\}^{1 / 4} \quad(u \leq t \leq x),
$$

whence

$$
N(\lambda) \leq \frac{1+o(1)}{(v-u)^{2} w}\left(\int_{u}^{x} e^{\lambda t}\{\min (t-u, v-u)\}^{1 / 2} d t\right)^{2}
$$


Clearly this is better than one obtains from Lemma 11.1, corresponding to the non-optimal choice $w(t)=1$. However it is not clear how to proceed if one hopes for an improvement on (11.3).

8) We may extend the useful range of the argument in $\S 12$ by applying it to a subset $S$ of the set

$$
\left\{\chi^{(j)}: 1 \leq j \leq N\right\}=T,
$$

say, chosen as follows. We delete $\chi_{1}$ and $\overline{\chi_{1}}$ from $T$, and put one of the remaining characters, $\psi_{1}$ say, into $S$, along with its conjugate. We then delete $\psi_{1} \chi_{1}, \psi_{1} \overline{\chi_{1}}, \overline{\psi_{1}} \chi_{1}$ and $\overline{\psi_{1} \chi_{1}}$, if present, from $T$. A further character, $\psi_{2}$ say, together with its conjugate, is now transferred from $T$ to $S$, and any of $\psi_{2} \chi_{1}, \psi_{2} \overline{\chi_{1}}$, $\overline{\psi_{2}} \chi_{1}$ and $\overline{\psi_{2} \chi_{1}}$ which are present in $T$ are deleted. The process is continued, and eventually results in a set $S$ for which

$$
3 \# S+2 \geq N
$$

and such that $\bar{\psi} \psi^{\prime} \neq \chi_{1}$ or $\overline{\chi_{1}}$ for $\psi, \psi^{\prime} \in S$. In analogy with Lemma 12.1 we then deduce that

$$
\# S \leq \frac{F\left(-\lambda_{2}\right)\left[F\left(-\lambda_{2}\right)-f(0) / 6\right]}{\left[F\left(\lambda-\lambda_{2}\right)-f(0) / 6\right]^{2}-F\left(-\lambda_{2}\right) f(0) / 6}+\varepsilon .
$$

If we know that $\lambda_{2}>\lambda_{1}$ we can use (16.7) and (16.8) to obtain non-trivial bounds for $N(\lambda)$, for values of $\lambda$ beyond those achievable in Lemma 12.1.

9 ) It would be nice to have a weighted version of Lemma 12.1, in the way that (11.4) is a weighted version of (11.5). Unfortunately, no neat way of achieving this seems available. The following argument however demonstrates that a little more can be squeezed out from the argument of $\S 12$. The reasoning there leads to

$$
\left\{\sum_{j \leq N} F\left(\lambda^{(j)}-\lambda_{1}\right)-N \frac{\phi}{2} f(0)\right\}^{2} \leq F\left(-\lambda_{1}\right)\left[F\left(-\lambda_{1}\right)-\frac{\phi}{2} f(0)\right]+\varepsilon .
$$

Thus either $N(1) \leq 4$, say, or

$$
\begin{aligned}
\left\{(N-5) F\left(\lambda-\lambda_{1}\right)+5 F\right. & \left.\left(1-\lambda_{1}\right)-N \frac{\phi}{2} f(0)\right\}^{2} \\
& \leq F\left(-\lambda_{1}\right)\left[F\left(-\lambda_{1}\right)-\frac{\phi}{2} f(0)\right]+\varepsilon .
\end{aligned}
$$

The latter inequality yields improved bounds for $N(\lambda)$ relative to Lemma 12.1, as soon as $\lambda>1$. Both the above possibilities would lead to reduced estimates for Linnik's constant. 


\section{References}

[1] D.A. Burgess, 'On character sums and L-series,II', Proc. London Math. Soc. (3), 13 (1963), 524-536.

[2] D.A. Burgess, 'The character sum estimate with $r=3$ ', J. London Math. Soc. (2), 33 (1986), 524-536.

[3] J.-R. Chen, 'On the least prime in an arithmetical progression', Sci. Sinica, 14 (1965), 1868-1871.

[4] J.-R. Chen, 'On the least prime in an arithmetical progression and theorems concerning the zeros of Dirichlet's L-functions', Sci. Sinica, 20 (1977), 529562 .

[5] J.-R. Chen, 'On the least prime in an arithmetical progression and two theorems concerning the zeros of Dirichlet's L-functions (II)', Sci. Sinica, 22 (1979), 859-889.

[6] J.-R. Chen, 'The exceptional set of Goldbach numbers (II)', Sci. Sinica, 26 (1983), 714-731.

[7] J.-R. Chen, and J.-M. Liu, 'On the least prime in an arithmetical progression (III) \& (IV)', Science in China, Ser. A, 32 (1989), 654-673 \& 792-807.

[8] H. Davenport, Multiplicative number theory (Markham, Chicago, 1967).

[9] S.W. Graham, 'Applications of sieve methods', Ph.D. Thesis, Univ. Michigan, 1977.

[10] S.W. Graham, 'An asymptotic estimate related to Selberg's sieve', J. Number Theory, 10 (1978), 83-94.

[11] S.W. Graham, 'On Linnik's constant', Acta Arith., 34 (1981), 163-179.

[12] A. Granville, and C. Pomerance, 'On the least prime in certain arithmetic progressions', J. London Math. Soc. (2), 41 (1990), 193-200.

[13] T.H. Gronwall, 'Sur les séries de Dirichlet correspondant à des charactères complexes', Rendiconti di Palermo, 35 (1913), 145-159.

[14] D.R. Heath-Brown, 'Siegel zeros and the least prime in an arithmetic progression', Quart. J. Math. Oxford Ser. (2), 41 (1990), 405-418.

[15] H. Heilbronn, 'Über den Primzahlsatz von Herrn Hoheisel', Math. Zeit., 36 (1933), 394-423.

[16] C. Hooley, 'The distribution of sequences in arithmetic progressions', Proc. Int. Cong. Math., Vancouver, 1974, Vol.I, 357-364. 
[17] M.N. Huxley, 'Large values of Dirichlet polynomials, III', Acta Arith., 26 (1974), 435-444.

[18] H. Iwaniec, 'On zeros of Dirichlet's L-series', Inventiones Math., 23 (1974), 97-104.

[19] M. Jutila, 'On two theorems of Linnik concerning the zeros of Dirichlet's L-functions', Ann. Acad. Sci. Fenn. Ser. A, 458 (1969), 32pp.

[20] M. Jutila, 'A new estimate for Linnik's constant', Ann. Acad. Sci. Fenn. Ser. A, 471 (1970), 8pp.

[21] M. Jutila, 'On Linnik's constant', Math. Scand., 41 (1977), 45-62.

[22] S. Knapowski, 'On Linnik's theorem concerning exceptional L-zeros', Publ. Math. Debrecen, 9 (1962), 168-178.

[23] E. Landau, 'Über die Klassenzahl imaginär-quadratischer Zahlkörper', Göttinger Nachrichten, (1918), 285-295.

[24] Yu.V. Linnik, 'On the least prime in an arithmetic progression. I. The basic theorem', Rec. Math. (Mat. Sbornik) N.S., 15(57) (1944), 139-178.

[25] Yu.V. Linnik, 'On the least prime in an arithmetic progression. II. The Deuring-Heilbronn phenomenon', Rec. Math. (Mat. Sbornik) N.S., 15(57) (1944), 347-368.

[26] K.S. McCurley 'Explicit zero-free regions for Dirichlet L-functions', $J$. Number Theory, 19 (1984), 7-32.

[27] R.J. Miech, 'A number-theoretic constant', Acta Arith., 15 (1969), 119-137.

[28] Y. Motohashi, 'On a density theorem of Linnik', Proc. Japan Acad. 51 (1977), 815-817.

[29] Y. Motohashi, 'Primes in arithmetic progressions', Inventiones Math., 44 (1978), 163-178.

[30] C.-D. Pan, 'On the least prime in an arithmetical progression', Sci. Record (N.S.), 1 (1957), 311-313.

[31] C.-D. Pan, 'On the least prime in an arithmetical progression', Acta Sci. Natur. Univ. Pekinensis, 4 (1958), 1-34.

[32] J. Pintz, 'Elementary methods in the theory of L-functions, V. The theorems of Landau and Page', Acta Arith., 32 (1977) 163-171.

[33] C. Pomerance, 'A note on the least prime in an arithmetic progression', $J$. Number Theory, 12 (1980), 218-223. 
[34] S.B. Stechkin, 'Zeros of the Riemann zeta-function', Math. Notes, 8 (1970), 706-711.

[35] E.C. Titchmarsh, 'A divisor problem', Rendiconti Palermo, 54 (1930), 414429.

[36] E.C. Titchmarsh, The theory of functions (Clarendon Press, Oxford, 19??).

[37] E.C. Titchmarsh, The theory of the Riemann Zeta-function (Clarendon Press, Oxford, 1951).

[38] P. Turán, 'On a density theorem of Yu.V. Linnik', Publ. Math. Inst. Hungar. Acad., 6 (1961), 165-179.

[39] P. Turán, 'On some recent results in the analytic theory of numbers', Number Theory Institute, 1969: Proc. Symp. Pure Math. XX, 359-374 (Amer. Math. Soc., Providence, RI, 1971).

[40] W. Wang, 'On the least prime in an arithmetic progression', Acta Math. Sinica, 29 (1986), 826-836. 